$\mathrm{LA}--9121-\mathrm{HS}$

DE82 007054
LA.9121-MS

UC-34

Issued: December 1981

\title{
Vacancy Behavior in a \\ Compressed fcc Lennard-Jones Crystal
}

Joe R. Beeler, Jr.*

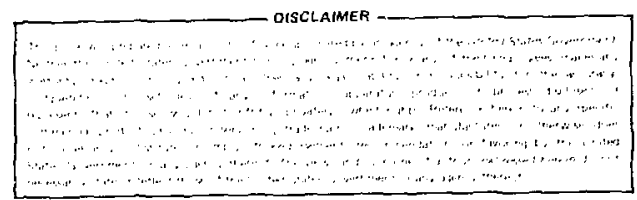

*Visiting Staff Member. Department of Materials Engineering, North Carolina State

University, Raleigh, NC 27650. 
VACANCY BEHAVIOK IN A COMPRESSED

fCC LENNARD-JONES CRYSTAL

by

Joe R. Beeler, Jr.

\begin{abstract}
This computer experiment study concerns the determination of the stable vacancy configuration in a compressed fcc Lennard-Jones crystal and the migration of this defect in a compressed crystal. Isotropic and uniaxial compression stress conditions were studied. The isotropic and uniaxial compression magnitudes employed were $0.94 \leqslant n \leqslant 1.5$, and $1.0 \leqslant n \leqslant 1.5$, respectively. The site-centered vacancy (SCV) was the stable vacancy configuration whenever cubic symmetry was present. This includes all of the j.sotropic compression cases and the particular uniaxial compression case $(n=\sqrt{2})$ that give a bcc structure. In addition, the SCV was the stable configuration for uniaxial compression $n<1.29$. The outof-plane split vacancy (SV-OP) was the stable vacancy configuration for uniaxial compression $1.29<\eta \leqslant 1.5$ and was the saddle-point configuration for SCV migration when the SCV was th:? stable form. For $n>1.20$, the $\mathrm{SV}-\mathrm{OP}$ is an extended cefect and, therefore, a saddle point for SV-OP migration could not be determined. The mechanisin for the transformation from the SCV to the SV-OP as the stable form at $\eta=1.29$ appears to be an alternating sign [101] and/or [011] shear process.
\end{abstract}

\title{
I. INTRODUCTION
}

This report describes computational results for the static equilibrium properties of a vacancy in a compressed face-centered cubic (fcc) Lennard-Jones crystal as a function of compression. The static equilibrium properties calculated were the displacement fields and configuration energies for a site-centered vacancy (SCV) and a split. vacancy (SV). These two vacancy configurations are described in Fig. 1. An SCV is produced by removing an atom from a normal atom 


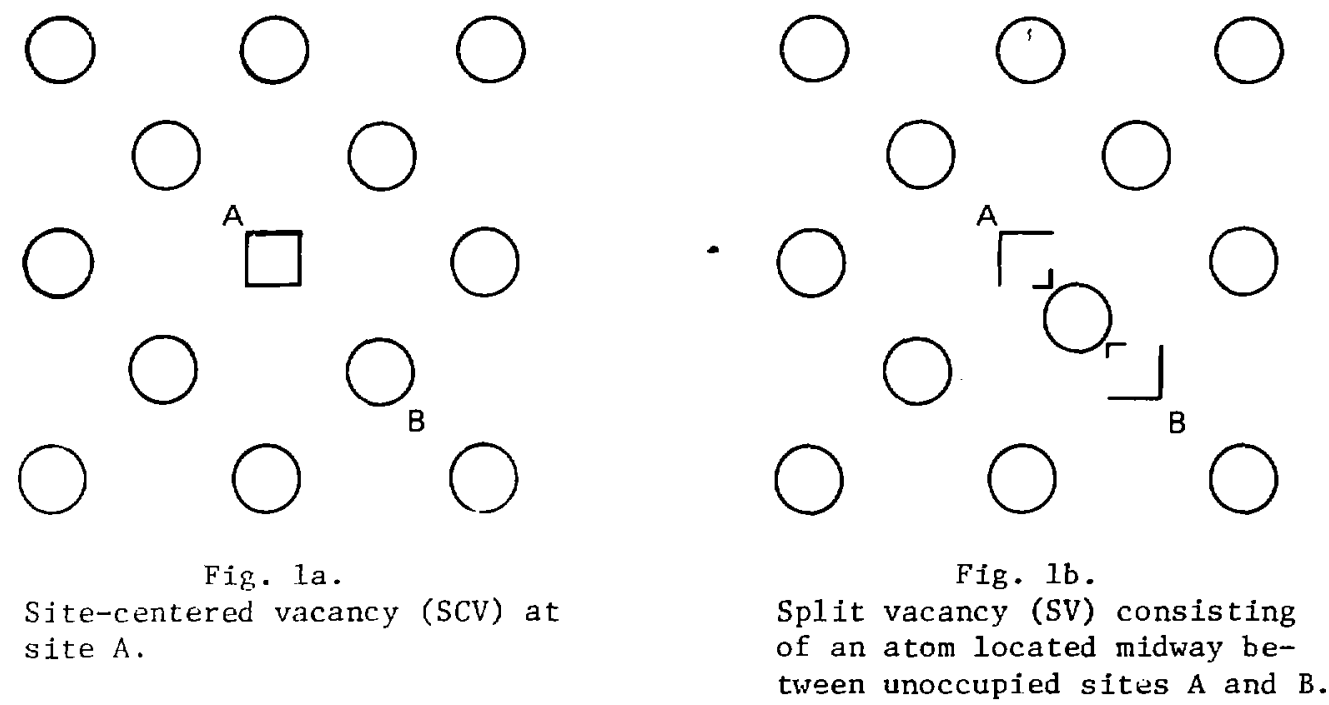

site $A$ in a crystal. An SV is produced by first creating an SCV at site $A$ and then moving the atom at one of the first-neighbor sites $B$ of $A$ to the point midway between $A$ and $B$. Two cases were considcred: (1) uniaxial compression along the [001] direction and (2) isotropic compression. The compression ( $n$ ) ranges considered were $1.0 \leqslant n \leqslant 1.5$, for uniaxial comp "ssion, and $0.97 \leqslant n \leqslant 1.5$ for isotropjc compression. The calculations were done using the MOL.MY program, a dynamical method computer experiment program written by B. L. Holian and G. K. Gtraub. A dynamical method program can be modified to compute static equilibrium properties of defects by adding a velocity damping term to the equations of motion. This modification was made in the MOLDY program to prnvide a capability for doing defect static equilibrium property calculations. Detailed explanations and illustrations of how to perform static equilibrium computations for defects in bcc, fcc, and hcp crystals are given by Beeler (1970) and by Beeler and Beeler (1979a, $h, c, d)$.

The present study was suggested by B. L. Holian and was made for the purpose of gaining background data for use in the interpretation of mass transport results obtained in dynamical method computer experiments on shock processes in an fcc Lennard-Jones crystal containing vacancy defects. These shock process simulations will be made using the MOLDY program. Guinan et al. (1977) showed that most of 
the dynamical attributes of interstitial diffusion in tungsten(m3), * observed in a fully dynamical simulation of interstitial migration in tungsten(m3), could be inferred from static equilibrium calculations of the displacement fields and configuration energies for the stable interstitial and interstitial migration saddlepoint configurations. This particular experie sce indicated that statip equilibrium calculations for the displacement fields and configuration energies for the stable vacancy and the vacancy migration saddle-point configurations in a compressed crystal should give valuable data for use in interpreting the results of dynamical method shock wave simulations for crystals containing vacancy defects.

In particular, one might expect that the migration energy for vacancy movement parallel to the uniaxial stress axis would differ significantly from that for vacancy movement perpendicular to the stress axis. In addition, one might expect that the displacement field details for vacancy movement during uniaxial compression would differ significantly from those for vacancy movement during isotropic compression. In this regard, one might expect that experimental data for defect motion obtained in nydrostatic pressure experiments need not be applicable in analyzing the results of shock wave experiments. The results described in this report confirm these particular expectations for face-centered telragonal (fct) and fcc Lennard-Jones crystals.

It was found that the stable vacancy configuration changes from an SCV to an SV at compression $n=1.29$ during uniaxial compression. This vacancy configuration change is a new result that has not been reported previously. In the case of isotropic compression, however, the SCV configuration is stable up to $\eta=1.5$. No calculations were done for $\eta>1.5$.

II. INTERATOMIC POTENTIAL AND COMPUTATIONAL CELL

The Lennard-Jones atom pair potential energy function used was

$$
V(r)=\varepsilon\left[\left(\frac{r_{0}}{r}\right)^{12}-2\left(\frac{r_{0}}{r}\right)^{6}\right],
$$

\footnotetext{
*Tungsten(m3) is a model for tungsten meta1, which consists of joining the Wilson and Bisson (1971) tungsten potential with the Johnson and Wilson (1972) tungsten potential at about $1.7 \AA$, with the Wilson-Bisson potentia' being used for atom separation distances less than $1.7 \AA$. The Wilson-Bisson and Johnson-Wilson potentials are referred to as tungsten(ml) and tungsten(m2), respectively.
} 
where $r_{0}=3.8666 \AA$ and $\varepsilon=9.10858 \times 10^{-3} \mathrm{eV}$. This potential was truncated to $V(r)=0$ at $r_{\text {cut }}=7.486592 \AA$.

The MOLDY computational cell used was a 500-site fcc crystallite with periodic boundary conditions. The cell was a cube with $\{100\}$ faces in the case of isotropic compression and a right rectangular parallelepiped with $\{100\}$ faces in the case of uniaxial compression. In each case there were five unit cells alor.g each Cartesian coordinate axis, each of which contained four atoms. This gives a total of 125 unit cells and 500 atom sites. For compression $n=1.0$, the lattice constant was $a_{0}=5.468198 \AA$. For uniaxial compression, the lattice constant along each of the directions [100] and [010] was $a_{0}$; but that along the [001] direction was $a_{0} ; n$, giving an fct crystal. An $x y-p l a n e$ in the fct crystal, in which the lattice constant along both [100] and [010] is $a_{0}$ for all $\eta$, will be called a bas 1 plane. The bascil plane half-lattice constant will be denoted as hlc(xy) and is equal to $a_{0} / 2$. The half-lattice constant along the z-axis will be denoted as hlc $(z)$ anu is equal to $a_{0} / 2 n$. For isotropic compression the lattice constant was $a_{0} /(\eta)^{1 / 3}$ for each of the three coordinate directions.

Atom site number and atom site coordinate maps for the 500-site computational cell are given in Fig. 2. Figure $2 a$ is an $x y-p l a n e$ map, which is applicable for cach odd-numbered (NZ) plane, and Fig. $2 b$ is an xy-plane map, which is applicable for each even-numbered (NZ) plane. The atom site coordinates are expressed in hlc and hlc(xy) for the $x$ - and $y$-directions and hlc(z) for the z-direction. The appropriate plane number (N\%), plane z-coordinate ( $z$ ), and site number (NS) range for each plane concerned are listed in a guide table located at the right side of each map. Given the coordinates $(x, y, z)$ of an atom site, $S$, one can find the associated computational cell site number, NS, in two steps: (1) use the z-coordinate to sclect the proper map section (the map section whose guide table lists the $z$-coordinate concerned, (2) use $(x, y)$ to locate site $S$ on the map. At the site location $(x, y)$ tile map gives an integer label $L$. The associated site number NS is given by

$$
\begin{array}{ll}
N S=100 \frac{\mathrm{NZ}-1}{2}+\mathrm{L} & (\text { for odd NZ) } \\
\mathrm{NS}=100 \frac{\mathrm{NZ}-2}{2}+\mathrm{L} & (\text { for even NZ) }
\end{array}
$$




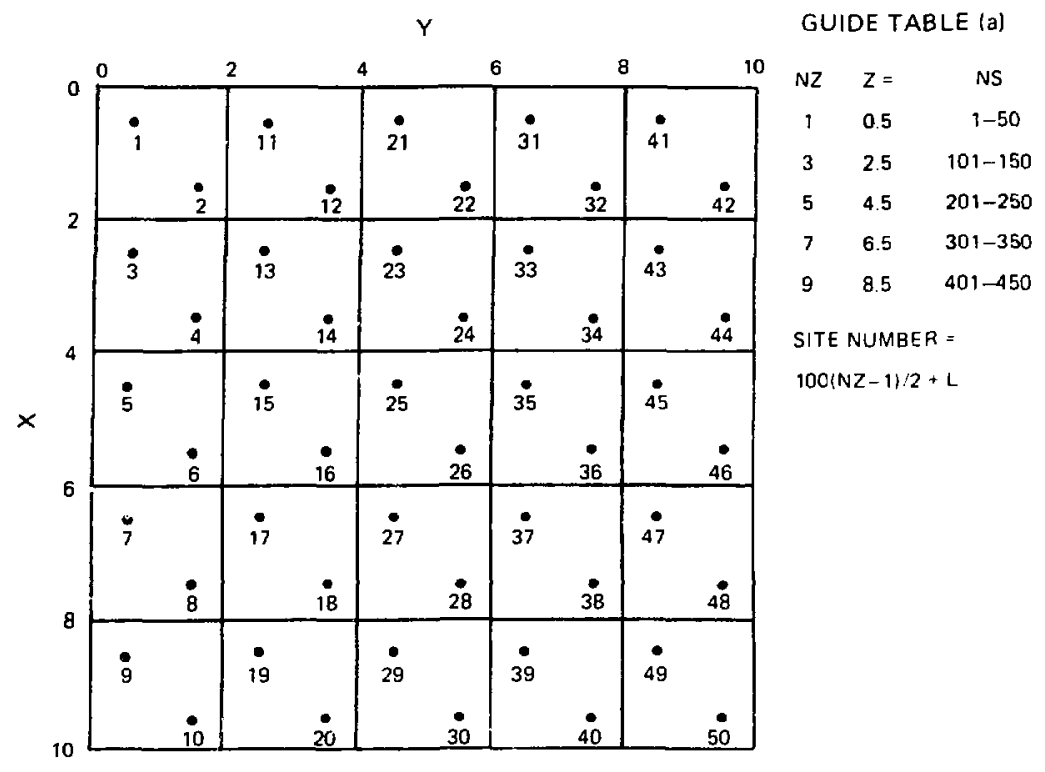

(a) Odd-numbered (NZ) Z-planes.

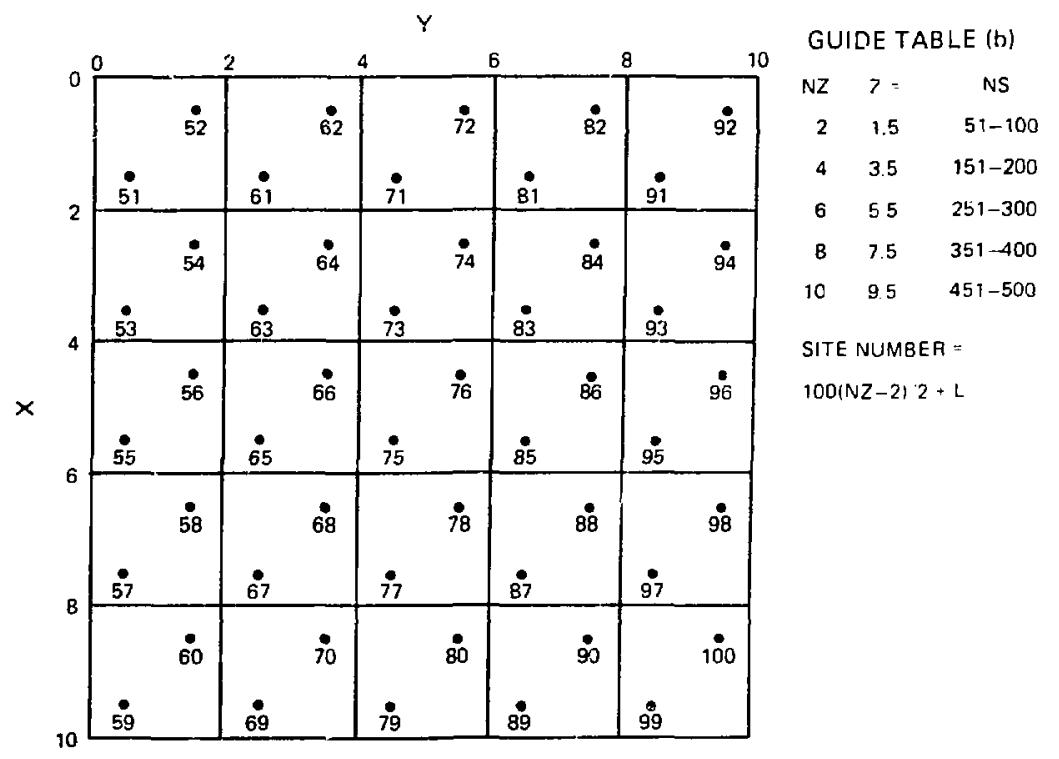

(b) Even-numbered (NZ) Z,-planes.

Fig. 2.

Atou site number and site coordinate naps for a MOLDY program 500-site fcc computational cell. 
When an fcc crystal is compressed uniaxially along the [001] direction, from $n=1.0$ to $n=\sqrt{2}$, the crystal structure changes from $\mathrm{fcc}$, at $n=1.0$, to bcc at $\eta=\sqrt{2}$. This change is described in Fig. 3. Figure 3a shows the atom sites in two adjacent fcc cubic unit cells. The unit of length is an hlc; hence, the edge length of the cubic unit cell is 2.0. Atom sites represented by open circles lie in the $x=0$ plane, those represented by filled circles lie in the $x=1.0$ plane, and those represented by hatched circles 1 ie in the $x=2.0$ plane. Note that the sites numbered 1-8 are at the corners of the right parallelepiped with a square base, and that site 9 is located at the center of this parallelepiped. The height of the parallelepiped is 2.0 and the edge length of its square base is $\sqrt{2}$. Figure $3 b$ shows sites $1-9$ only and ignores the other sites depicted in Fig. $3 a$. Figure $3 b$ defines a tetragonal unit cell for an fcc crystal and contains two atoms, one-eighth of an atom at each of the eight corners and one atom at the center of the cell. It is clear that a uniaxial compression $n=\sqrt{2}$ along the [001] direction reduces the parallelepiped of height 2.0 , shown in Fig. $3 b$, to the cube of edge length $\sqrt{2}$, shown in Fig. 3c. One recognizes Fig. $3 c$ as being a cubic unit cell for a bcc crystal. This bcc unit cell contains two atoms, oneeighth of an atom at each of the eight corners and one atom at the center of the cel1.

One can use Figs. $3 b$ and $3 c$ to relate the direction of a line segment connecting the centers of two given atoms in the fcc cell, at $\eta=1.0$, to the direction of the line segment connecting the centers of these same two atoms in the bcc cell at $n=\sqrt{2}$. For example, the direction from site 9 to site 3 in the fcc cell (Fig. 3b) is [101]. Figure 3 shows that the direction froil site 9 to site 3 in the bcc cell is [111]. Hence, [101] fcc goes into [111] bcc. Table I lists siteto-site line segments and the corresponding directions for these segments in the fcc cell and the bcc cell. Perhaps the most important of these correspondences are (1) $[100]_{\mathrm{fcc}}$ goes into $[110]_{\mathrm{bcc}}$, (2) $[010]_{\mathrm{fcc}}$ goes into $[\overline{1} 10]_{\mathrm{bcc}}$, and (3) $[001]_{\text {fcc }}$ goes into $[001]_{\text {bcc }}$.

Table II lists the relative coordinates $\mathrm{RC}_{\mathrm{n}}$ and coordination number $\mathrm{CN}_{\mathrm{n}}$ for sites in each of the first ten neighbor shells of a given site $S$ in an fcc crystal and in a bcc crystal. Given a site $S$ with coordinates $(x, y, z)$, and a neighboring site $S^{\prime}$ with coordinates $\left(x^{\prime}, y^{\prime}, x^{\prime}\right)$, the relative coordinates of $S^{\prime}$ with respect to $s$ are $x_{r}=\left(x^{\prime}-x\right), y_{r}=\left(y^{\prime}-y\right)$, and $z_{r}=\left(z^{\prime}-z\right)$. In terms of relative coordinates, any given neighbor shell may consist of two or more subshf.11s, each corresponding to a different set of relative coordinates. For 


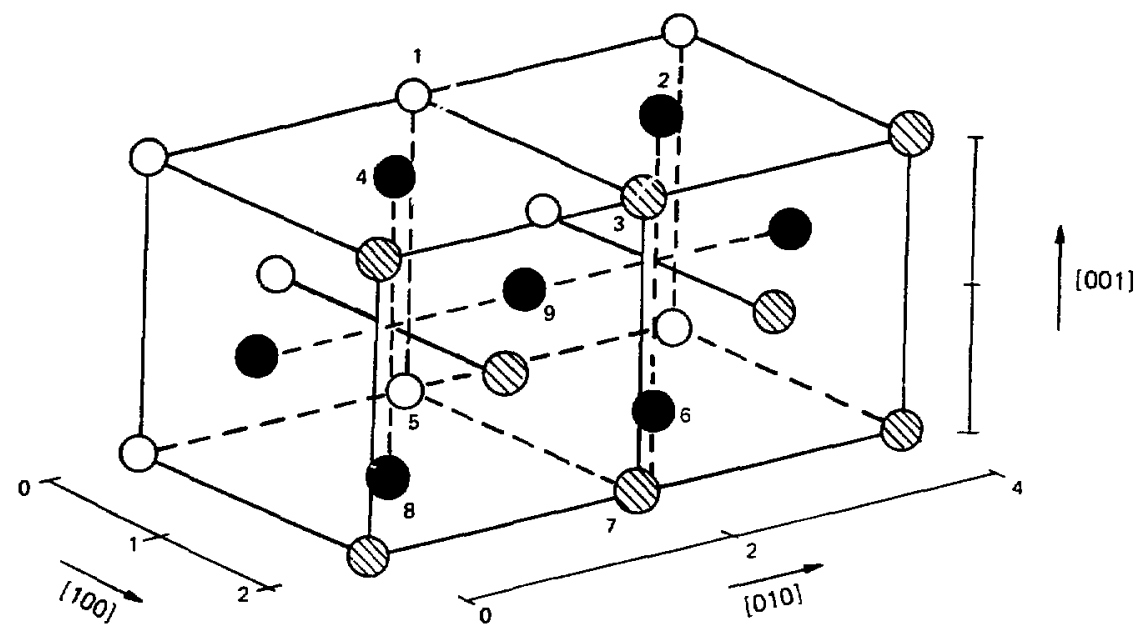

(a) Two cunic unit cells for an fcc crystal $(n=1.0)$.
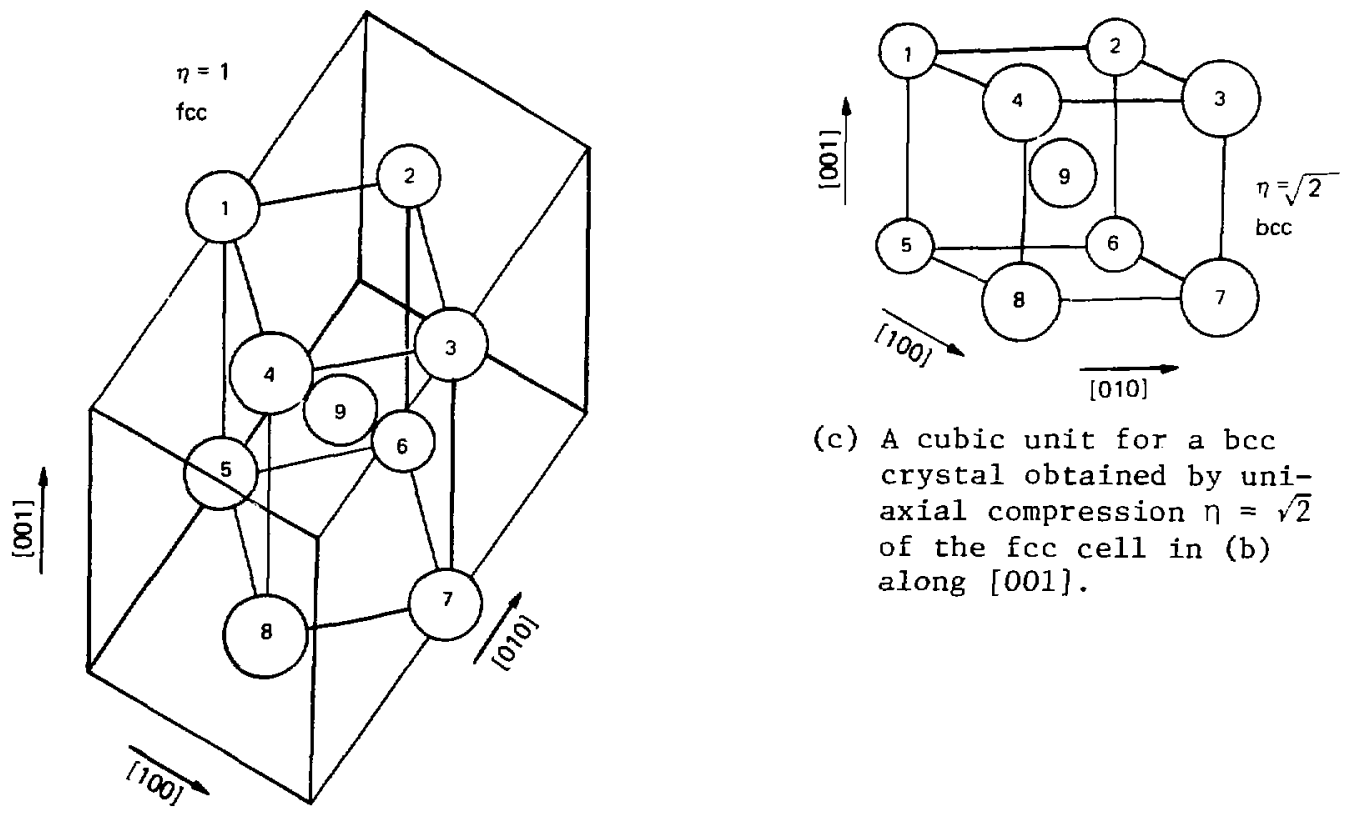

(c) A cubic unit for a bcc crystal obtained by uniaxial compression $\eta=\sqrt{2}$ of the fcc cell in (b) along [001].

(b) A tetragonal unit cell for an fcc crystal $(\eta=1.0)$.

Fig. 3.

Transition from an fcc crystal to a bcc crystal via uniaxial compression along [001]. 
TABLE I

Correspondence between the direction of a site-to-site segment in the fcc crystal $(n=1.0)$ and that for this segment in the bcc crystal occurring at $n=\sqrt{2}$. Site numbers defired in Fig. 3 .

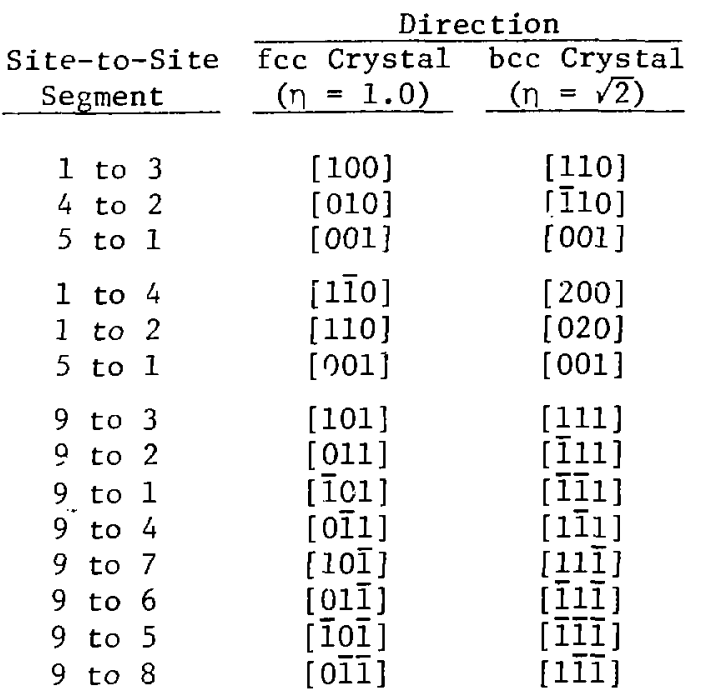

example, the sum of the squares of the relative coordinates for a site in the I0th neighbor shell in a bcc crystal is 27 , i.e., $r_{10}^{2}=27$. There are two relative coordinate sets which give $r_{10}^{2}=27$; one is $( \pm 3, \pm 3, \pm 3)$ and the other is $( \pm 5, \pm 1, \pm 1)$. Also given in Table $I I$ is the ratio $r_{n} / r_{1}$ of the $n-t h$ neighbor shell radius to the radius $r_{1}$ of the first neighbor shell. This ratio can be used to determine from what initial fcc subshell each member of a bcc subshell evolved during uniaxial compression from $\eta=1.0$ to $\eta=\sqrt{2}$. Table III gives the neighbor subshell transformation correspondence for going from an fcc crystal $(n=1.0)$ to a bcc crystal $(n=\sqrt{2})$ via uniaxial compression along the [001] direction. This table was prepared to show how

each of the first six neighbor shells in the bcc crystal evolved from particular subshells in the fcc crystal.

One procedure for constructing Table III is as follows. At uniaxial compression $\eta=\sqrt{2}, h l c(x y)=\sqrt{2} h l c(b c c)$, hlc $(b c c)$ being the bcc crystal half-lattice constant. From Table I or from Fig. 3, it follows that, at this compression, $[100]_{f c t}$ is coaxial with $[110]_{b c c},[010]_{f c t}$ is coaxial with $[\overline{1} 10]_{b c c}$, and

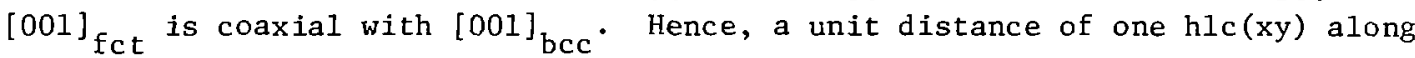
$[100]_{\mathrm{fct}}$ is equal to a distance of $\sqrt{2} \mathrm{hlc}(\mathrm{bcc})$ along $[110]_{\mathrm{bcc}}$, a distance of one $\mathrm{hlc}(\mathrm{xy})$ along $[010]_{\mathrm{fct}}$ is equa]. to a distance of $\sqrt{2} \mathrm{hlc}(\mathrm{bcc})$ along $[\overline{1} 10]_{\mathrm{bcc}}$, and one $h l c(z)$ along $[001]_{f c t}$ is equal to one $h l c(b c c)$ along [001] bcc. This demortstrates that the vectors $[100]_{\mathrm{fct}}$ and $[110]_{\mathrm{bcc}}$ are equal, as are the vectors $[010]_{f c t}$ and $[\overline{1} 10]_{b c c}$ and the vectors $[001]_{f c t}$ and $[001]_{b c c}$. Consequently, the vector $r_{r}=\left(x_{r}, y_{r}, z_{r}\right)$ from $S$ to $S^{\prime}$ in the fct coordinate system is 


$$
\begin{aligned}
r_{r}(f c t) & =x_{r}[100] f c t \\
& =x_{r}[110]_{b c c}[010]_{f c t}+y_{r}\left[z_{r}[001]\right]_{f c t} \\
b c c & +z_{r}[001]_{b c c} .
\end{aligned}
$$

In the bcc coordinate system, this vector is, from Eq. (3b),

$$
r_{r}(b c c)=\left(x_{r}-y_{r}\right)[100]_{b c c}+\left(x_{r}+y_{r}\right)[010] b c c+z_{r}[001]_{b c c} .
$$

\section{TABLE II}

Neighbor shell relative coordinates $R C_{n}$, radius $r_{n}$, and coordination number $C_{n}$ for each of the first ten neighbor shells in an fcc and a bcc crystal. The relative coordinates are expressed in half-lattice constants ( $h l c$ ), $n$ is the neighbor shell number (index).

fcc Crystal Neighbors

$$
\begin{array}{r}
\hline \text { n } \\
1 \\
2 \\
3 \\
4 \\
5 \\
6 \\
7 \\
8 \\
9 \\
10 \\
10
\end{array}
$$

\begin{tabular}{crrr}
$\mathrm{RC}_{\mathrm{n}}$ & & $\mathrm{r}_{\mathrm{n}}^{2}$ & $\mathrm{C}_{\mathrm{n}}$ \\
\cline { 1 - 1 }$(1,1,0)$ & & 2 & 12 \\
$(2,0,0)$ & 4 & 6 \\
$(2,1,1)$ & 6 & 24 \\
$(2,2,0)$ & 8 & 12 \\
$(3,1,0)$ & 10 & 24 \\
$(2,2,2)$ & 12 & 8 \\
$(3,2,1)$ & 14 & 48 \\
$(4,0,0)$ & 16 & 6 \\
$(3,3,0)$ & 18 & 12 \\
$(4,1,1)$ & 18 & 24 \\
$(4,2,0)$ & 20 & 24
\end{tabular}

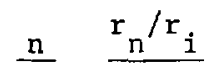

11.

21.4142

$3 \quad 1.732$

42.0

$5 \quad 2.2361$

$6 \quad 2.4495$

$7 \quad 2.6458$

$8 \quad 2.8284$

\begin{tabular}{|c|c|c|c|}
\hline $\mathbf{n}$ & $\mathrm{RC}_{\mathrm{n}}$ & $\mathrm{r} \frac{2}{n}$ & $C_{n}$ \\
\hline 1 & $(1,1,1)$ & 3 & 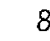 \\
\hline 2 & $(2,0,0)$ & 4 & 6 \\
\hline 3 & $(2,2,0)$ & 8 & 12 \\
\hline 4 & $(3,1,1)$ & 11 & 24 \\
\hline 5 & $(2,2,2)$ & 12 & 8 \\
\hline G & $(4,0,0)$ & 16 & 6 \\
\hline 7 & $(3,3,1)$ & 19 & 24 \\
\hline 8 & $(4,2,0)$ & 20 & 24 \\
\hline 9 & $(4,2,2)$ & 24 & 24 \\
\hline 10 & $(3,3,3)$ & 27 & 8 \\
\hline 10 & $(5,1,1)$ & 27 & 24 \\
\hline
\end{tabular}

93.0

$10 \quad 3.1623$ bcc Crystal Neighbors 
Neighbor subshell transformation correspondence in going from an fcc crystal $(n=1.0)$ to a bcc crystal $(n=\sqrt{2})$ by means of uniaxial compression along the [001] direction.

\begin{tabular}{|c|c|c|c|c|c|}
\hline \multicolumn{3}{|c|}{ fcc Crystal } & \multicolumn{3}{|c|}{ bcc Crystal } \\
\hline $\begin{array}{l}\text { Number } \\
\text { of Sites }\end{array}$ & $\begin{array}{c}\text { Subshel1 } \\
\text { Type }\end{array}$ & $\begin{array}{c}\text { Shell } \\
\text { No. }\end{array}$ & $\begin{array}{l}\text { Number } \\
\text { of Sites }\end{array}$ & $\begin{array}{c}\text { Subshell } \\
\text { Type }\end{array}$ & $\begin{array}{l}\text { She1l } \\
\text { No. }\end{array}$ \\
\hline 8 & $\langle 101\rangle$ & 1 & 8 & $\langle 111\rangle$ & 1 \\
\hline 4 & $\langle 110\rangle$ & 1 & 4 & $<200\rangle$ & 2 \\
\hline 2 & $<002>$ & 2 & 2 & $<002\rangle$ & 2 \\
\hline 4 & $\langle 200\rangle$ & 2 & 4 & $<220\rangle$ & 3 \\
\hline 8 & $\langle 112\rangle$ & 3 & 8 & $<202>$ & 3 \\
\hline 16 & $\langle 121\rangle,\langle 211\rangle$ & 3 & 16 & $<311\rangle$ & 4 \\
\hline 8 & $\langle 103\rangle$ & 5 & 8 & $\langle 113\rangle$ & 4 \\
\hline 8 & $<202\rangle$ & 4 & 8 & $<222>$ & 5 \\
\hline 4 & $\langle 220\rangle$ & 4 & 4 & $\langle 400\rangle$ & 6 \\
\hline 2 & $\langle 004\rangle$ & 8 & 2 & $<004\rangle$ & 6 \\
\hline
\end{tabular}

Relations (3) and (4) show that the relative coordinates $\left(x_{r}, y_{r}\right.$, $z_{r}$ ) in the fct crystal transform to bcc crystal relative coordinates, at $\eta=\sqrt{2}$, as follows:

$$
\begin{aligned}
& x_{r}(b c c)=\left(x_{r}-y_{r}\right), \\
& y_{r}(b c c)=\left(x_{r}-y_{r}\right), \\
& z_{r}(b c c)=z_{r} .
\end{aligned}
$$

Table III also can be constructed by computing the distance between a site $S$ and each of its neighbors after uniaxial compression $\eta=\sqrt{2}$ along [001] and taking the ratio of this distance to $r_{1}(b c c)$. Because there is a unique value of $r_{n} / r_{1}$ for each neighbor shell $n$, the distance ratio obtained indicates the proper neighbor shell in the bcc crystal. Let $\left(x_{r}, y_{r}, z_{r}\right)$ be the relative coordinates of site $S^{\prime}$ with respect to site $S$ when $\eta=1.0$. The distance from $S$ to $S^{\prime}$ is then

$$
\begin{aligned}
& d\left(S \text { to } S^{\prime}\right)=\left(x_{r}^{2}+y_{r}^{2}+z_{r}^{2} / \eta^{2}\right)^{1 / 2}, \\
& d\left(S \text { to } S^{\prime}\right)=\left(x_{r}^{2}+y_{r}^{2}+z_{r}^{2} / 2.0\right)^{1 / 2}, n=\sqrt{2} .
\end{aligned}
$$


From Fig. 3c, the bcc first neighbor distance $r_{1}$ (DCc) is the length of the siteto-site segment from site 9 to site 3 . Using $\mathrm{Eq}$. (7) one obtains $\mathrm{r}_{1}(\mathrm{bcc})=$ 1.224745 and the unit of length is $\mathrm{hlc}(\mathrm{xy})$. As an example, consider the $\mathrm{fcc}$ first neighbor subshell whose relative coordinates are $( \pm 1,0 \pm 1)$. For each of these neighbors $d\left(S\right.$ to $\left.S^{\prime}\right)=1.224745$ when $n=\sqrt{2}$, and, hence, each of them also is a first neighbor in the bcc crystal. In contrast, the sites in the $( \pm 1, \pm 1,0)$ fcc f:-st neighbor subshell give $d\left(S\right.$ to $\left.S^{\prime}\right)=1.414214$ when $n=\sqrt{2}$. Thj.s gives a ratio $\mathrm{d}\left(\mathrm{S}\right.$ to $\left.\mathrm{S}^{\prime}\right) / \mathrm{r}_{1}=1.154701$. According to Table $\mathrm{II}$ this ratio indicates the second neighbor shell in the bcc crystal.

\section{PERFECT CRYSTAL ENERGY}

The perfect crysial energy is plotted in Fig. $4 \mathrm{a}$ as a function of compression. The upper curve is for uniaxial compression and the lower curve is for isotropic compression. The data base for these plots is given by Tables IV and V. In the case of uniaxial compression, the lowest energy occurred for $7=3.10$ (see Table IV). The lowest energy occurred near $\eta=1.10$ in the case of isotropic compression. A blowup of the crystal energy for isotropic compression (Fig. 4b) shows a relative minimum at $\eta=1.06$ and a discontinuity at $\eta=1.102$ (see Appendix A). This discontinuity is caused by the truncation of the potential function at the atom separation distance $r_{\text {cut }}=7.486592 \AA$. For $n<1.1021107$, each atom interacts only with its first, second, and third neighbors. At $n=1.1021107$, interaction with fourth neighbors occurs, in addition, and causes the sharp drop pictured in Fig. 4a. As indicated in Table I, there are 12 fourth neighbors. A second discontinuity would occur at $\eta=1.540250$ due to the addition of $f$ ifth neighbor interactions. There are $24 \mathrm{fifth}$ neighbors, as indicated in Table I.

Discontinujiies also occur in the crystal energy curve for uniaxial compression at $\eta=1.0694,1.1769$, and 1.4608 but are not detectable in Fig. 4a because the density of data points is too sparse to reveal them. The first of these discontinuities is caused by the addition of [202] and [022] fourth neighbor interactions which involve eight of the twelve fourth neighbors. The second discontinuity is caused by additional interactions with [103] and [013] fifth neighbors which involve only 8 of the 24 fifth neighbors. The third discontinuity is caused by the addition of two [004] eighth neighbors. The neighbor shell reference used here for the additional interaction atoms denotes the shells in which the particular atoms concerned are located when $\eta=1.0$. 

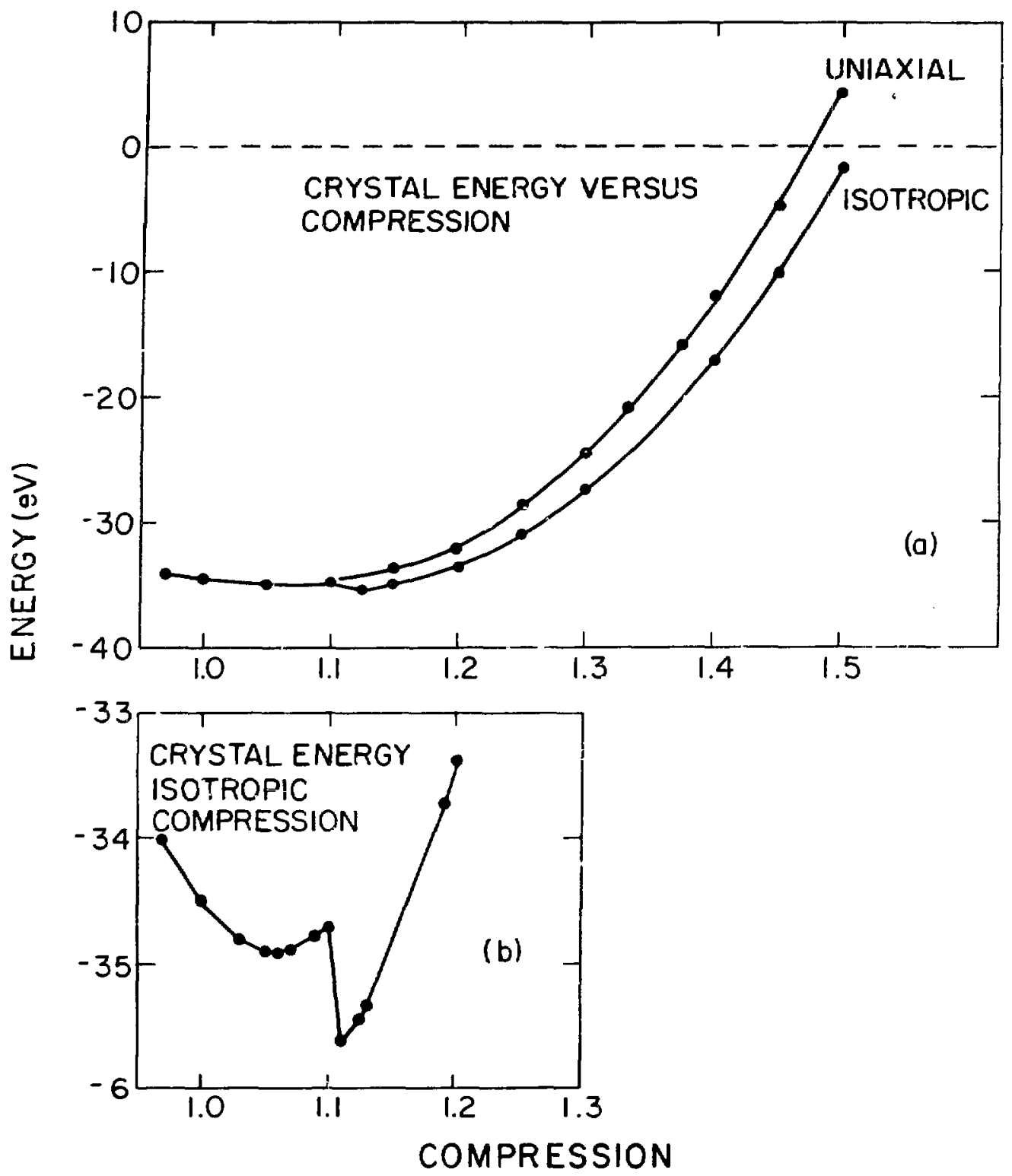

Fig. 4 .

(a) Crys:al energy vs compression for uniaxial and isotropic compression of an fcc Lennard-Jones crystal.

(b) Blowup of the crystal energy for isotropic compression in the neighborhood of the energy discontinuity at $\eta=1.1021107$. 
TABLE IV

Crystal energy $E^{-x}(P C)$ for a perfect 500atom fct r.rystal obtalned by untaxial compression of a 500-atom fcc LennardJones crystal along [001].

\begin{tabular}{lrr} 
Compression & $E^{\mathrm{X}}(\mathrm{PC}), \mathrm{eV}$ & \multicolumn{1}{c}{$\mathrm{E}^{\mathrm{a}}(\mathrm{PC}), \mathrm{eV}$} \\
\hline 1.0 & -34.490068 & -.068980 \\
1.05 & -34.789679 & 069579 \\
1.10 & -34.984722 & -0.069969 \\
1.15 & -33.604673 & -0.067209 \\
1.20 & -31.994802 & -0.063990 \\
1.25 & -28.768570 & -0.057537 \\
1.30 & -24.494780 & -0.048930 \\
$1.33 \ldots$ & -21.042101 & -0.042084 \\
1.375 & -16.016528 & -0.032033 \\
1.40 & -12.040060 & -0.024080 \\
1.414. & -10.524629 & -0.021049 \\
1.45 & -4.815714 & -0.009631 \\
1.50 & 4.205555 & -0.008411
\end{tabular}

\section{TABLE $Y$}

Crystal energy $E^{X}(P C)$ for a perfect 500atom $\mathrm{fcc}$ crystal obtained by isotropic compression of a 500-atom fcc LennardJones crvstal.

\begin{tabular}{|c|c|c|}
\hline Compression & $E^{X}(P C), e V$ & $E^{a}(P C), e V$ \\
\hline 0.9703 & -3.992791 & -0.067986 \\
\hline 1.0 & -34.490068 & -0.068980 \\
\hline 1.0303 & -34.809020 & -0.069618 \\
\hline 1.05 & -34.905721 & -0.069811 \\
\hline 1.0612 & -34.919161 & -0.069838 \\
\hline 1.07 & -34.908016 & -0.069816 \\
\hline 1.0927 & -34.786729 & -0.069573 \\
\hline 1.10 & -34.718546 & -0.069437 \\
\hline 1.11 & -35.642621 & -0.071285 \\
\hline 1.1249 & -35.442990 & -0.070886 \\
\hline 1.13 & -35.359072 & -0.070718 \\
\hline 1.15 & -34.956531 & -0.069913 \\
\hline 1.1910 & -33.733827 & $-0.06 ?$ \\
\hline 1.20 & -33.390618 & $-0.066 \ldots$ \\
\hline 1.2250 & -32.282121 & -0.064564 \\
\hline 1.25 & -30.945933 & $-0.06189 ?$ \\
\hline 1.2597 & -30.360609 & -0.060721 \\
\hline 1.30 & -27.521446 & -0.055043 \\
\hline 1.3310 & -24.859952 & -0.049720 \\
\hline 1.33 & -24.642028 & -0.049284 \\
\hline 1.375 & -20.315167 & -0.040630 \\
\hline 1.40 & -17.306071 & -0.034612 \\
\hline 1.45 & -10.291589 & -0.020583 \\
\hline 1.50 & -1.849878 & -0.003700 \\
\hline
\end{tabular}

Table VI gives the number of atom pair interactions (NPI) per atom as a function of compression. The calculations involved are given in Appendix A. NPI is constant over most of the range of interest, $1.102<n \leqslant 1.5$, for isotropic compression. For uniaxial compression, NPI is constant over a slightly smaller range, $1.177<n<$ 1.46. Within these two compression ranges there are no discontinuities in the crystal energy.

\section{TABLE VI}

The number of atom pair interactions (NPI) per atom as a function of compression.

(a) 1.PI for Selucted Compression Values

\begin{tabular}{|c|c|c|}
\hline$\pi$ & $\begin{array}{c}\text { Uniaxial } \\
\text { Compression }\end{array}$ & $\begin{array}{r}\text { Isotr } \\
\text { Compre }\end{array}$ \\
\hline 1.0 & 42 & 42 \\
\hline 1.05 & 42 & 42 \\
\hline 1.10 & 50 & 42 \\
\hline 1.15 & 50 & 54 \\
\hline 1.20 & 58 & 54 \\
\hline 1.25 & 58 & 54 \\
\hline 1.30 & 58 & 54 \\
\hline 1.33 & 58 & 54 \\
\hline 1.375 & 58 & 54 \\
\hline 1.40 & 58 & 54 \\
\hline 1.414 & 58 & 54 \\
\hline 1.45 & 58 & 54 \\
\hline 1.50 & 60 & 54 \\
\hline
\end{tabular}

(b) NPI for Compression Ranges

\begin{tabular}{ll} 
Untaxial Compression & NPI \\
\cline { 1 - 1 } $1.0<n<1.069365$ & 42 \\
$1.069365<n<1.176887$ & 50 \\
$1.176887<n<1.460798$ & 58 \\
$1.460798<n<1.50$ & 60 \\
Isotropic Compression & NPI \\
\hline $1.0 \leqslant n<1.102111$ & 42 \\
$1.102111<\eta<1.5$ & 54
\end{tabular}


IV. VACANCY CONFIGURATION AND RELAXATION ENERGY

A. Introduction

The configuration energy $E^{C}(D)$ for a particular defect $D$ is defined as

$$
E^{C}(D)=E^{x}(D)-E^{x}(P C),
$$

where $E^{x}(P C)$ is the energy of the perfect crystal configuration and $E^{x}$ (D) is the energy of the crystal after the defect configuration has been established in the crystal. In the case of a vacancy, $\mathrm{E}^{\mathrm{x}}(\mathrm{D})$ is the crystal energy obtained when an atom is extracted from a normal atom site and all other atoms are allowed to change their positions in response to any unbalanced forces introduced by the extraction. Atom positional change occurring as a result of introducing a defect is called relaxation. In general, introduction of a defect involves (1) an initial removal and/or creation of atom pair interactions, and (2) a consequent relaxation of atom positions. The defect configuration energy obtained when only the initial atom pair interaction changes are accounted for and relaxation is not considered, is called the unrelaxed configuration energy $E^{c}(D)$ ur . The difference,

$$
E^{r}(D)=E^{C}(D)_{u r}-E^{c}(D),
$$

between $E^{C}(D)$ ur and $E^{C}(D)$ is called the relaxation energy of the defect.

The magnitude of the relaxation energy relative to that of the unrelaxed configuration energy reflects the intensity of the relaxation process for a given defect. In this context, the relaxation intensity $I_{r}(D)$ for a defect $D$ will be defined as

$$
I_{r}(D)=\left|E^{r}(D) / E^{c}(D) u r\right|
$$

The migration energy $E^{\mathrm{II}}(\mathrm{D})$ for a particular defect $D$ is defined as

$$
E^{m}(D)=E^{x}(S P)-E^{X}(D),
$$

where $E^{X}$ (SP) is the crystal energy when the saddle-point configuration for defect $D$ migration has been introduced. Determination of the saddle-point configuration 
for defect migration is discussed in detail by Beeler and Beeler (1979d). In computer experiment work, an estimate of the vacancy formation energy $E^{f}(V)$ commonly is obtained using the recipe

$$
E^{f}(V)=E^{c}(V)+E^{a}(P C),
$$

where $E^{a}(P C)$ is the potentiai energy per atom (cohesive energy) for a perfect crystal. $E^{C}(V)$ is the increase in the crystal energy which occurs when an atom is removed from a normal site in a perfect crystal. $\mathrm{E}^{\mathrm{a}}(\mathrm{PC})$ is the decrease in crystal energy which occurs when the removed atom is relocated on the crystal surface at an irterior corner in a surface ledge. The rationale for this recipe is discussed b; Damask and Dienes (1963). Hirth (1972) gives a careful theoretical discussion of the considerations involved in a defect-formation energy computation. In the case of an SCV,

$$
E^{c}(V)_{u r}=-2 E^{a}(P C)
$$

and, from Eq. (9),

$$
E^{r}(V)=E^{c}(V) \text { ur }-E^{c}(V)
$$

Relations (9) and (13), together with (12), give

$$
E^{f}(S C V)=-\left[E^{a}(P C)+E^{r}(S C V)\right]
$$

for an SCV. For an SV, one has

$$
E^{r}(S V)=E^{c}(S V)-E^{r}(S V)+E^{a}(P C) .
$$

As mentioned previously, an fcc crystal that is subjected to uniaxial compression $\eta$ along the [001] direction becomes an fct crystal with lattice constant $a$ along the [100] and [010] directions and lattice constant $c=a / \eta$ along the [001] direction. Two types of atom migration occur in a tetragonal crystal. One of these is atom migration in a basal plane, called in-plane migration, and 
the other is that in which an atom moves from one basal plane to another adjacent basal plane, called out-of-plane migration. Similarly, there are two linds of split vacancies in a tetragonal crystal, the in-plane split vacancy, SV-IP, and the out-of-plane split vacancy, SV-OP. The present work indicates that the stable vacancy configuration is the SCV for uniaxial compression $n<1.29$, and changes to the SV-OP for $\eta>1.29$.

When the SCV is the stable configuration, in-plane migration in a tetragonal crystal with a [001] tetragonal axis proceeds as shown schematically in Figs. $5 a, b$, and $c$. One of the first-neighbor atoms, $B$, of the initially vacant site $A$, moves to site A, thereby filling the original vacancy and learing a vacancy at site $B$. The saddle-point configuration is the SV-IP shown in Fig. 5b. In this instance the crystal energy change profile versus the moving atom position $r_{C}$ on the reaction coordinate line between sites $A$ and $B$ has a single peak at $r^{*}$ midway between $A$ and $B$, as shown in Fig. 6a.

Out-of-plane migration of an SCV in a tetragonal crysta1 with a [001] tetragonal axis involves a first-neighbor atom at a site $B$ in an adjacent basal plane moving into the initially vacant site $A$. In this instance the saddle point can be either simple or complex. In the simple case the saddle-point configuration is the SV-OP shown in Fig. 5d, and the associated crystal energy change profile is like that shown in Fig. 6a. A pair of saddle-point configurations exists, in the complex case, wherein the moving atom is positioned a distance $\mathrm{d}^{*}$ on ej.ther side of the midpoint. The crystal energy change profile versus $\mathbf{r}_{c}$ exhibits two peaks; one at $r_{1}^{*}$ and the other at $r_{2}^{*}$, as shown in Fig. 6b. The distance from the midpoint to either $r_{1}^{*}$ or $r_{2}^{*}$ is $d^{*}$. The valley between the two peaks normally is shallow, being only about $4 \%$ of the peak height. Because of this standard result for a twin-peak saddle point, the crystal energy for the SV-OP midpoint configuration can be used as an estimate of the crystal energy for the saddle-foint configuration. A detailed account of how to compute complete crystal energy profiles, such as those shown in Fig. 6, is given by Beeler and Beeler (1979d).

When the SV-OP is the stable vacancy configuration, the saddle-point configuration probably is complex. A schematic description of the crystal energy change profile for SV-OP migration is given in Fig. 7. This figure is based on the assumption that the SCV is the midpoint configuration. Past experience indicates that $E^{c}(S P)$ should be only slightly larger than $E^{c}(S C V)$. 


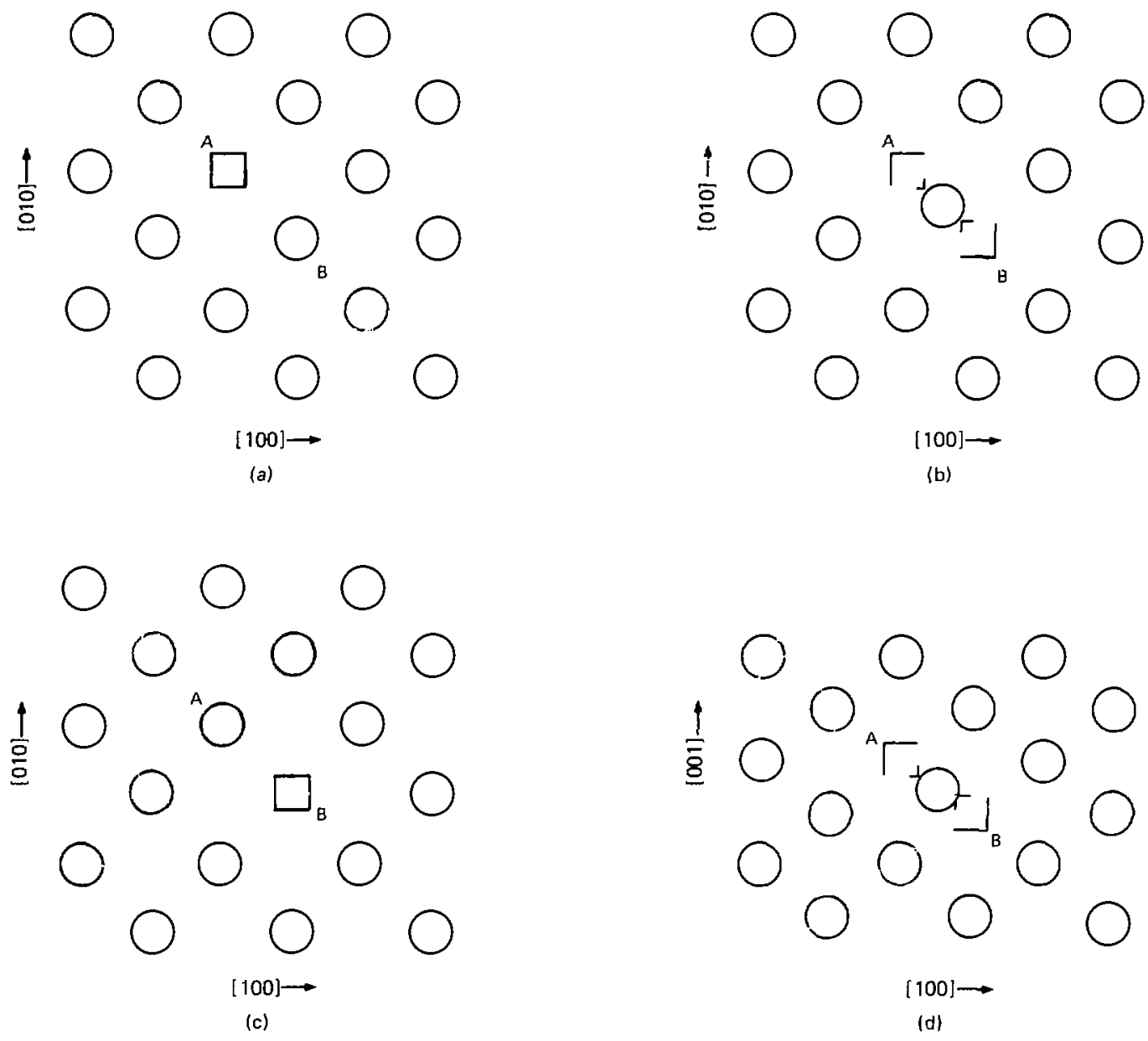

Fig. 5.

(a), (b), and (c) show migration of an SCV from site A to site B. The SV in (b) is the saddle-point configuration. This sequence pertains either to SCV migration in an fCC crystal or to SCV migration in a basal plare of an fct crystal. An out-of-plane split vacancy (SV-OP) in an fct crystal is shown in (d). An in-plane split vacancy (SV-IP) in an fct crystal is shown in (b). 


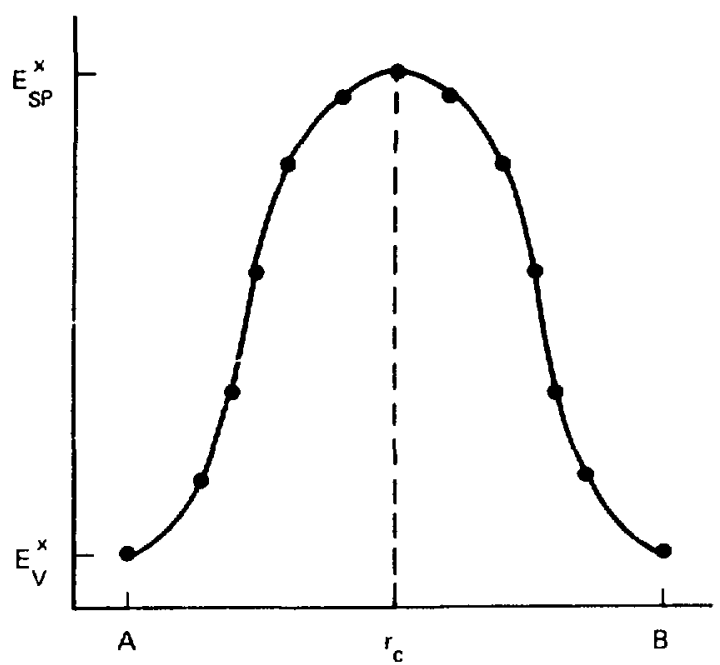

Fig. 6a.

Crystal energy profile for SCV migration in the case of a simple saddlepoint configuration.

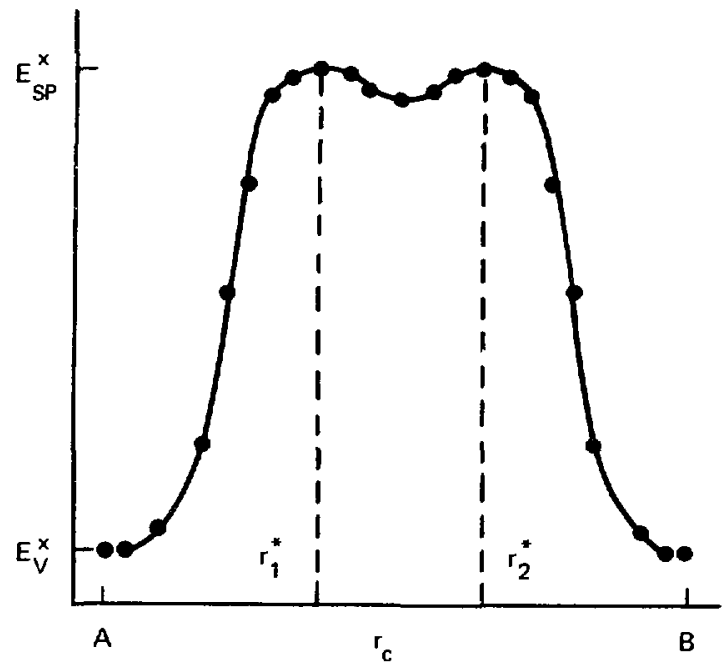

Fig. 6b.

Crystal energy profile ror SCV migration in the case of a complex saddlepoint configuration.

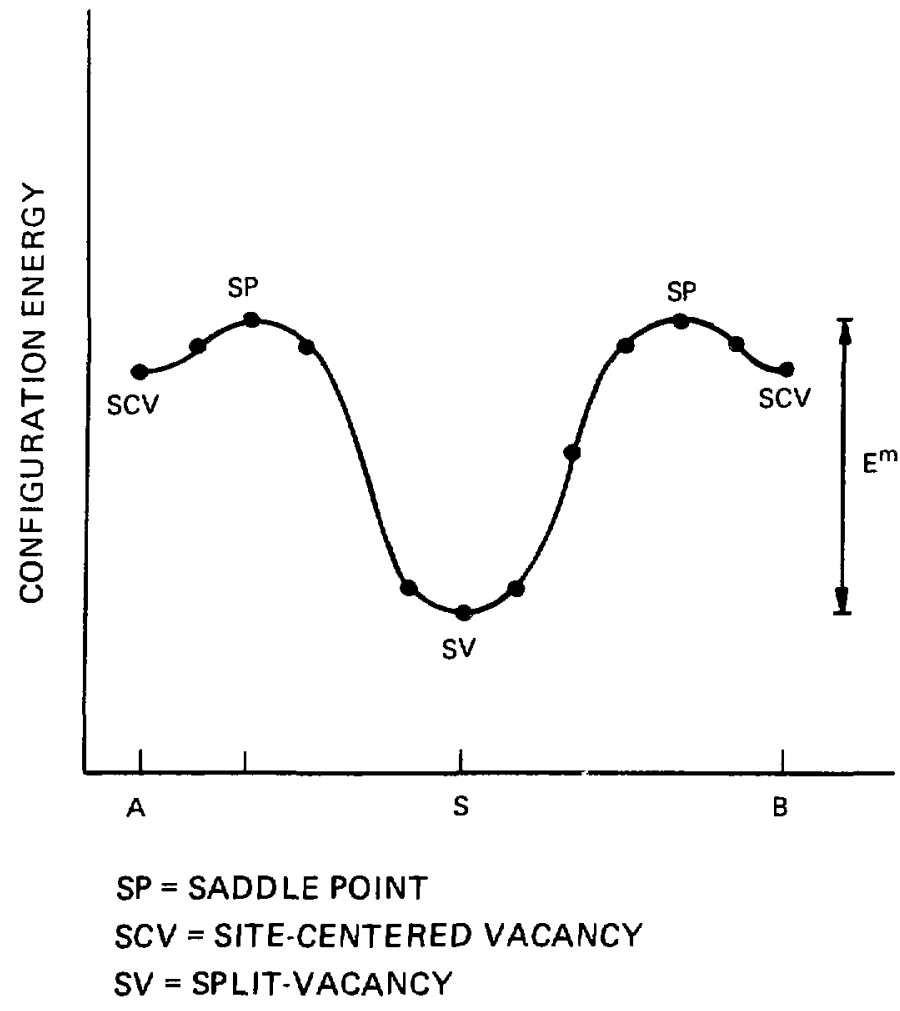

Fig. 7.

Configuration energy profile for SV migration. 


\section{B. Vacancy Configuration and Relaxation Energy}

1. Site-Centered Vacancy. The SCV configuration energy is plotted in Fig. 8 as a function of compression. The upper curve pertains to isotropic compressiun and the lower curve to uniaxial compression. In the case of isotropic compression, $E^{c}(\mathrm{SCV})$ is positive for $\eta<1.46$ and negative for $1.46 \leqslant n \leqslant 1.5$. For uniaxial compression, $E^{c}(S C V)$ is positive for $\eta<1.27$ and negative for $1.27 \leqslant n \leqslant$ 1.5. A negative configuration energy, in the case of a vacancy, means that the relaxation energy is larger than twice the magnitude of the binding energy per atom $\mathrm{E}^{\mathrm{a}}(\mathrm{PC})$ in a perfect crystal. As shown in Fig. 4, compression increases the crystal energy and there is a compression level at which the atom bonding is so weak that removing an atom actually lowers the crystal energy by affording neighboring atoms the freedom to move to positions at which they are more tightly bound than they are in their perfect crystal positions. This occurs at $\eta=1.27$ and $\eta=1.46$, respectiveiy, for unfaxial and isotropic compression.

The SCV relaxation energy is shown in Fig. 9. The relaxation energy increases monotonically with isotropic compression. In contrast, the relaxation energy rises to a maximum at about $\eta=1.36$ and then falls rapidly for $\eta>1.36$ when the crystal is compressed uniaxially. The compression regime associated with the relaxation energy peak during uniaxial compression corresponds closely to that.for a negative vacancy formation energy for that type of compression. In this context it is interesting to note that the atom displacement magnitudes also reach a peak within the compression regime 1.30-1.375. This feature is described by Figs. 25-35 in Sec. V.

Figure 10 allows one to compare the variations in the SCV configuration and relaxation energies with compression and also gives the perfect crystal binding energy per atom $E^{a}(P C)$ as a function of compression. Note that the relaxation energy exceeds the configuration energy for $n>1.24$. The configuration energy vanishes at $\eta=1.27$ and the $E^{r}(\mathrm{SCV})$ and $\mathrm{E}^{\mathrm{a}}(\mathrm{PC})$ curves show that $\mathrm{E}^{\mathrm{r}}(\mathrm{SCV})=$ $-2 \mathrm{E}^{\mathrm{a}}(\mathrm{PC})$ at $\eta=1.27$.

Figure 11 describes $E^{c}(S C V), E^{r}(S C V)$, and $E^{a}(P C)$ as functions of isotropic compression. Because the isotropically compressed crystal is more stable than the uniaxially compressed crystal (see ${ }^{\mathrm{T}} \mathrm{ig} .4$ ), the relaxation energy for the isotropic case is small compared with that for the uniaxial case. A relaxation energy peak, such as that seen in Fig. I0 for uniaxial compression, does not occur for $1.0 \leqslant n \leqslant 1.5$ in the isotropic compression calculations. 


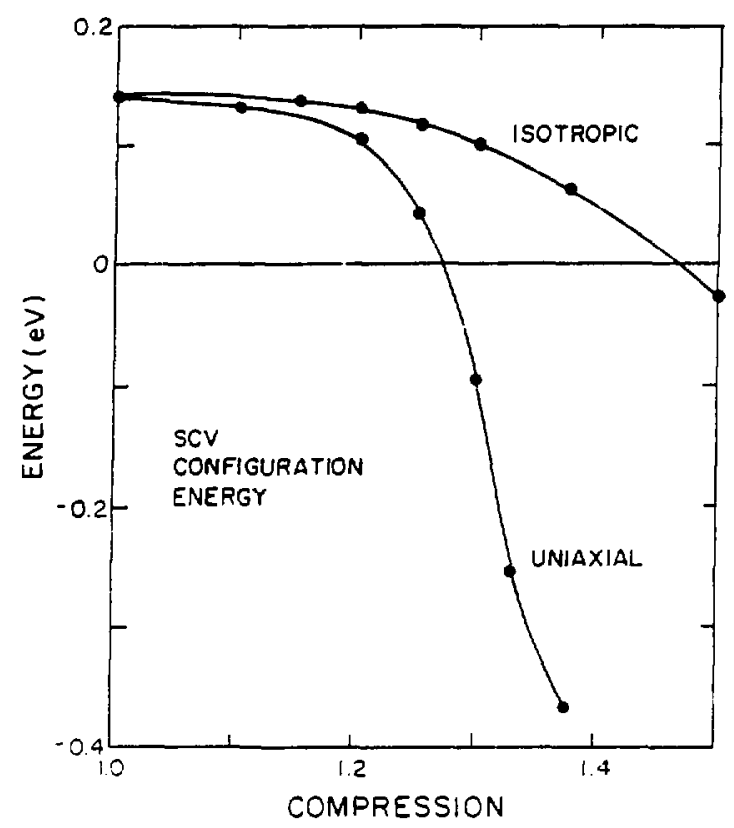

Fig. 8 .

SCV configuration energy vs compression.

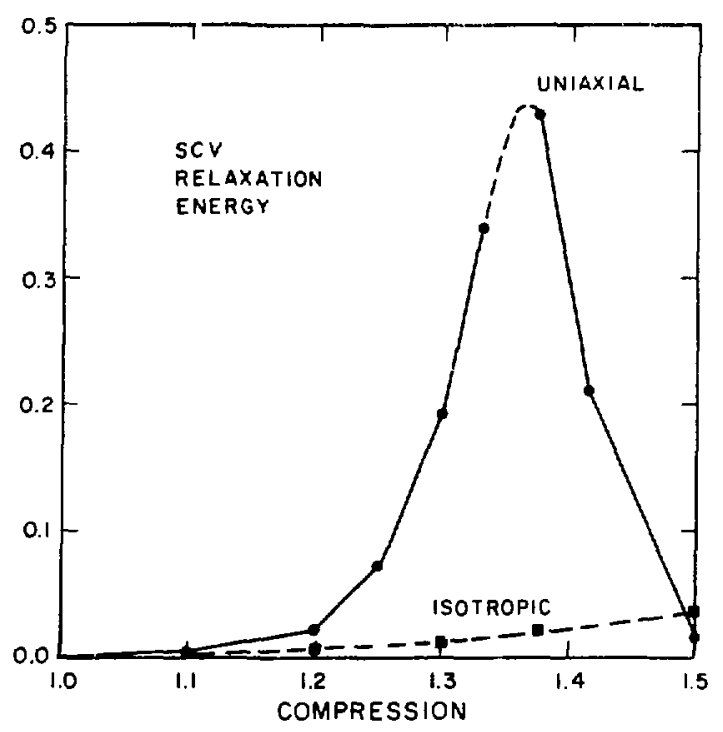

Fig. 9.

SCV relaxation energy vs compression.

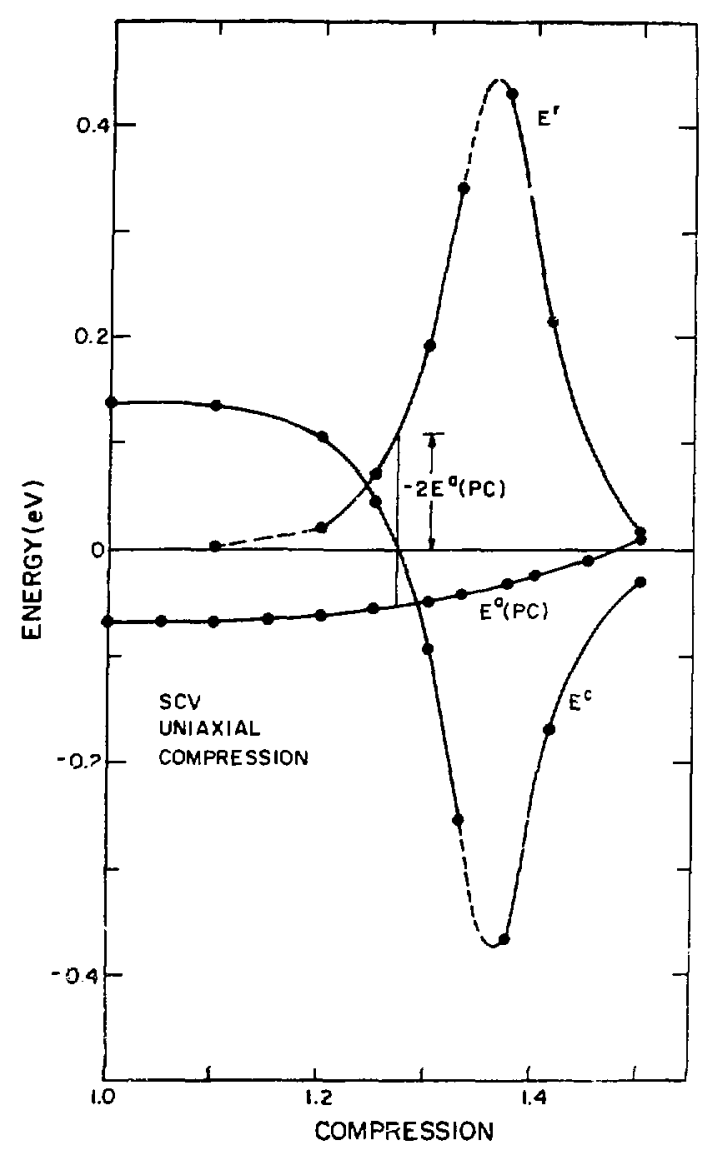

Fig. 10.

Composite plot of $\mathrm{E}^{\mathrm{C}}$ (SCV), $\mathrm{E}^{\mathrm{r}}$ (SCV), and $E^{a}(P C)$ for uniaxial compression. 


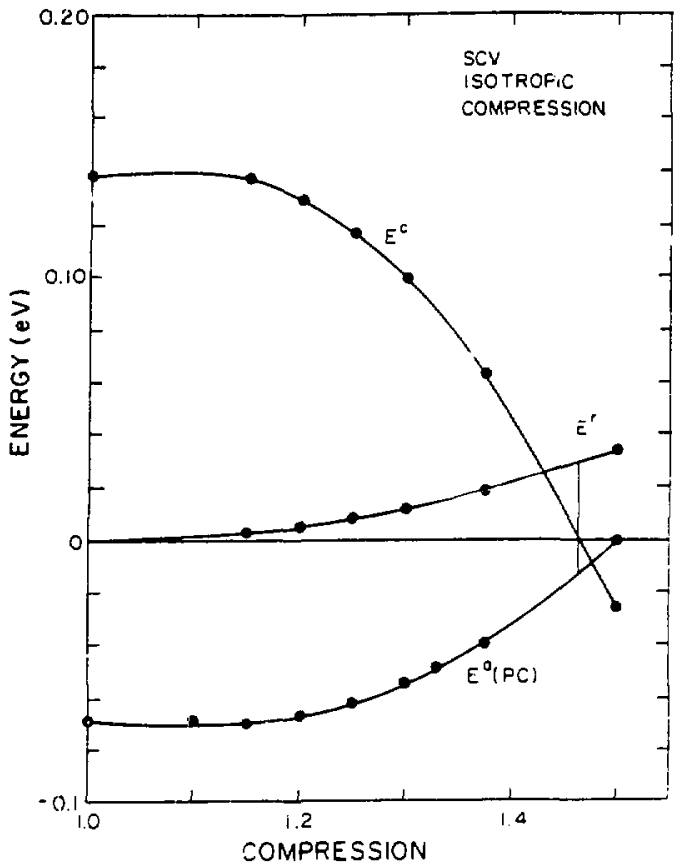

Fig. 11.

Composite plot of $\mathrm{E}^{\mathrm{C}}(\mathrm{SCV}), \mathrm{E}^{\mathrm{r}}(\mathrm{SCV})$, and $\mathrm{E}^{\mathrm{a}}(\mathrm{PC})$ for isotropic compression.

Tables VII and VIII contain the :iumerical data upon which the discussion of the SCV was tased. One aspect of these data which has not been discussed is the relaxation intensity $I_{r}$ (SCV). Table VII shows that the uniaxial compression regime for which $I_{r}(S C V)$ exceeds unity also corresponds to that in which $E^{r}(S C V)$ exceeds $-2 E^{a}(P C)$ and in which $\mathrm{E}^{\mathrm{a}}(\mathrm{PC})$ is negative. In the case of isotropic compression, $I_{r}(\mathrm{SCV})$ exceeds unity only for $1.47 \leqslant n \leqslant 1.5$, as is shown in Table IX. The $\mathrm{E}^{\mathrm{c}}$ (SCV) $\mathrm{ur}$ and $\mathrm{E}^{\mathrm{r}}$ (SCV) values used to prepare Table IX were read from plots of the data given in Table VIII.
TABLE VII

Uniaxial Compression: Site-centered vacancy energles expressed in $\mathrm{eV}$. $\mathrm{E}^{\mathrm{X}}(\mathrm{V})$ is the crystal energy, $E^{\mathrm{X}}(\mathrm{V})_{\mathrm{ur}}$ is the unrelaxed crystal energy, and $E^{r}(V)$ is the vacancy relaxation energy. $E^{C}(V)$ and $E^{C}(V)$ ur are the vacancy configuration energy and unrelaxed configuration energy, respectively.

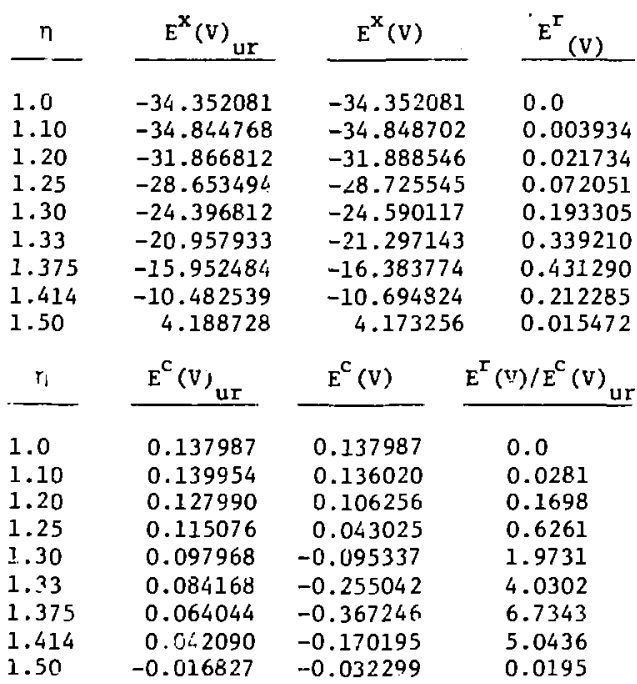

TABLE VIII

Isotropic Compression: Site-centered vacancy energies expressed in eV. $\mathrm{E}^{\mathrm{X}}(\mathrm{V})$ is the crystal energy, $E^{X}(V)_{u r}$ is the unrelaxed crystal energy, and $E^{T}(V)$ is the vacancy relaxation energy. $E^{C}(V)$ and $E^{C}(V)$ ur are the vacancy configuration energy and unrelaxed configuration energy, respectively.

\begin{tabular}{|c|c|c|c|}
\hline$n$ & $E^{x}(v)_{u r}$ & $E^{x}(V)$ & $E^{r}(V)$ \\
\hline $\begin{array}{l}1.0 \\
1.15 \\
1.20 \\
1.25 \\
1.30 \\
1.375 \\
1.50\end{array}$ & $\begin{array}{r}-34.352081 \\
-34.816905 \\
-33.256892 \\
-30.822105 \\
-27.411318 \\
-20.233915 \\
-1.842477\end{array}$ & $\begin{array}{r}-34.352081 \\
-34.819528 \\
-33.261809 \\
-30.830005 \\
-27.422759 \\
-20.252108 \\
-1.876274\end{array}$ & $\begin{array}{l}0.0 \\
0.002623 \\
0.004917 \\
0.007900 \\
0.011441 \\
0.018193 \\
0.033797\end{array}$ \\
\hline$n$ & $E^{C}(v)_{u r}$ & $E^{c}(V)$ & $E^{r}(V) / E^{c}(V) u r$ \\
\hline $\begin{array}{l}1.0 \\
1.15 \\
1.20 \\
1.25 \\
1.30 \\
1.375 \\
1.50\end{array}$ & $\begin{array}{l}0.137987 \\
0.139626 \\
0.133726 \\
0.123828 \\
0.110128 \\
0.081252 \\
0.007401\end{array}$ & $\begin{array}{r}0.137987 \\
0.137003 \\
0.128809 \\
0.115928 \\
0.098687 \\
0.063059 \\
-0.026396\end{array}$ & $\begin{array}{l}0.0 \\
0.0188 \\
0.0368 \\
0.0638 \\
0.1039 \\
0.2239 \\
4.5665\end{array}$ \\
\hline
\end{tabular}


TABLE IX

$I_{r}(S C V)$ for isotropic compression 1.46 $\leqslant n \leqslant 1.50$.

$\begin{array}{llll}n & I_{r}(S C V) & & I_{r}(S C V) \\ 1.46 & 0.80 & 1.49 & 2.46 \\ 1.47 & 1.07 & 1.50 & 4.57 \\ 1.48 & 1.48 & & \end{array}$

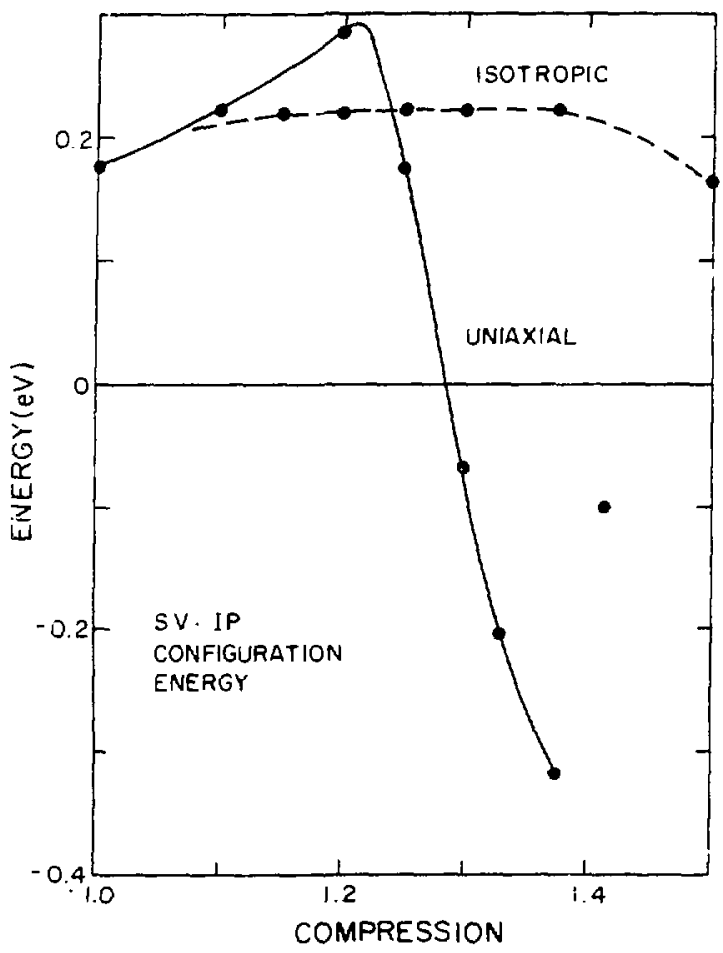

Fig. 12.

SV-IP configuration energy vs compression.

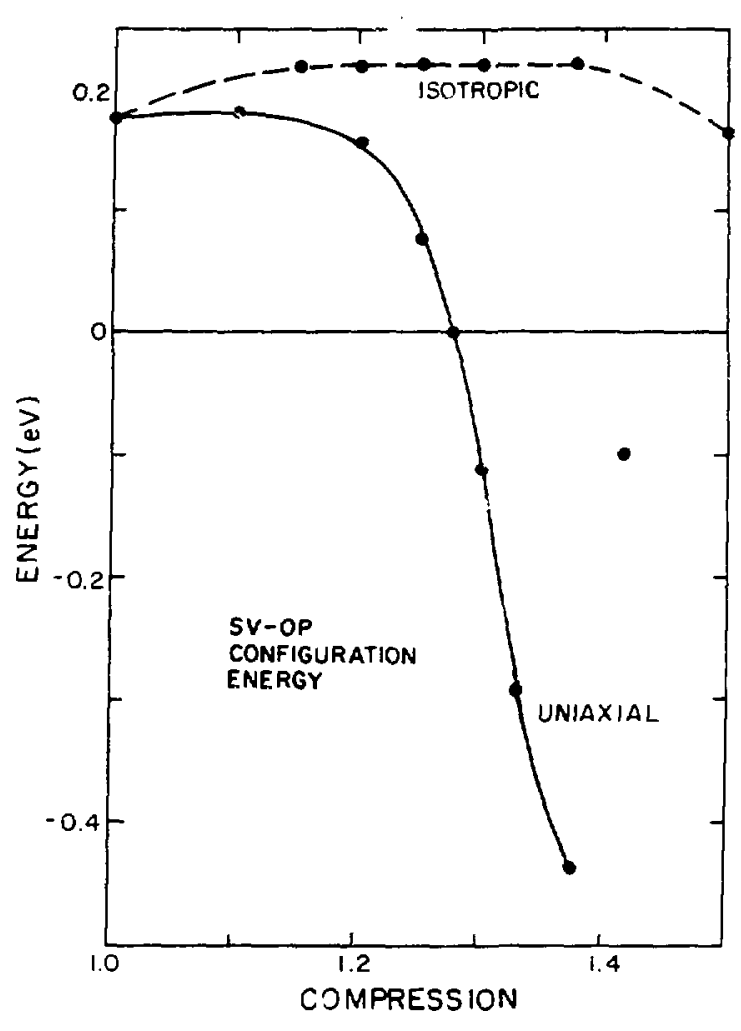

Fig. 13.

SV-OP configurjtion energy vs compres. sion.

2. Split Vacancy. As indicated in Fig. 5, there are two types of $\mathrm{SV}$ in a tetragonal crystal; one is the SV-IP described in Fig. $5 b$ and the other is the SV-OP described in Fig. 5d. The SV-IP axis lies in a tasal plane, whereas the $\mathrm{SV}-\mathrm{OP}$ axis runs from one basal plane to another. The SV-IP and SV-OP configuration energies are plotted versus unaxial compression in Figs. 12 and 13, respectively. Each of these figures also shows the SV configuration energy for isotropic compression. These and other figures in this section are based on the numerical data listed in Tables X-XIII. As shown in Fig. 14 and Table XII, the relaxed SV-IP and SV-OP have nearly the same 
TABLE X

Untaxlal Compression: In-plane mode splitvacancy energles expressed in eV. $E^{X}(V)$ is the crystal energy, $E^{x}(V)$ ur is the unrelaxed crystal energy, and $E^{r}(V)$ is the vacancy relaxation energy. $E^{C}(V)$ and $E^{C}(V)$ ur are the vacancy configuration energy and unrelaxed configuration energy, respectively.

\begin{tabular}{|c|c|c|c|}
\hline$n$ & $E^{X}(V)_{u r}$ & $E^{X}(V)$ & $E^{r}(V)$ \\
\hline $\begin{array}{l}1.0 \\
1.10 \\
1.20 \\
1.25 \\
1.30 \\
1.33 \\
1.375 \\
1.50\end{array}$ & $\begin{array}{r}-34.262591 \\
-34.619240 \\
-31.384385 \\
-27.982088 \\
-23.488438 \\
-19.862219 \\
-14.590136 \\
6.616828\end{array}$ & $\begin{array}{r}-34.313054 \\
-34.757901 \\
-31.707631 \\
-28.5931 .45 \\
-24.564352 \\
-21.247055 \\
-16.336243 \\
3.003331\end{array}$ & $\begin{array}{l}0.050463 \\
0.138661 \\
0.323246 \\
0.611057 \\
1.075914 \\
1.384836 \\
1.746107 \\
3.613497\end{array}$ \\
\hline$\pi$ & $E^{c}(V) u r$ & $E^{c}(V)$ & $E^{\mathbf{r}}(V) / E^{c}(V)_{u r}$ \\
\hline $\begin{array}{l}7.0 \\
1.10 \\
1.20 \\
1.25 \\
1.30 \\
1.33 \\
1.375 \\
1.50\end{array}$ & $\begin{array}{l}0.227477 \\
0.365482 \\
0.610417 \\
0.786482 \\
1.006342 \\
1.179882 \\
1.426392 \\
2.411271\end{array}$ & $\begin{array}{r}0.177014 \\
0.226821 \\
0.287171 \\
0.175425 \\
-0.069572 \\
-0.204954 \\
-0.319715 \\
-1.202224\end{array}$ & $\begin{array}{l}0.2218 \\
0.3794 \\
0.5295 \\
0.7769 \\
1.0691 \\
1.1737 \\
1.2241 \\
1.4986\end{array}$ \\
\hline
\end{tabular}

TABLE XI

Uniaxial Compression: Out-of-plane more splitvacancy energles expressed in $\mathrm{eV}$. $\mathrm{E}^{\mathrm{X}}(\mathrm{V})$ is the crystal energy, $E^{X}(V)$ ur is the unrelaxed crystal energy, and $\mathrm{E}^{\mathrm{r}}(\mathrm{V})$ is the vacancy relaxation energy. $E^{C}(V)$ and $E^{C}(V)$ ur are the vacancy configuration energy and unrelaxed configuration energy, respectively.

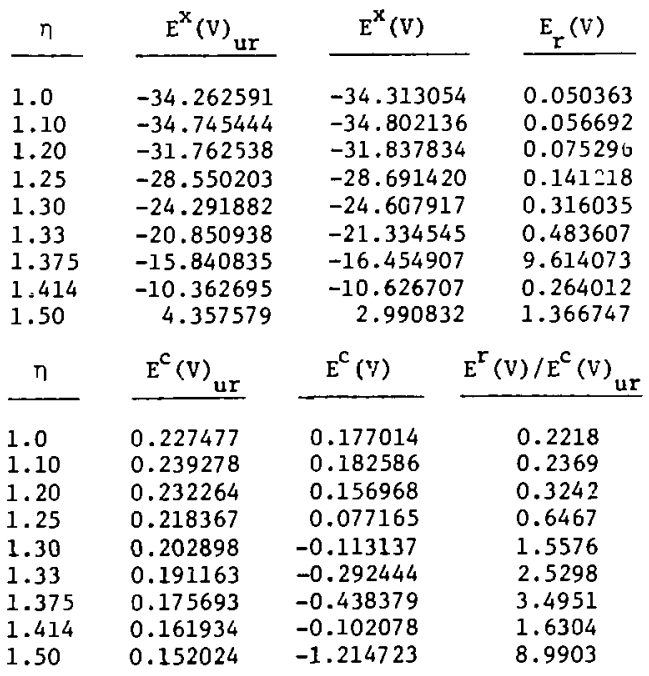

TABLE XII

Uniaxial Compression: The difference $E^{C} I P(V)-E^{C}{ }_{O P}(V)$ between the in-plane and the out-of-plane mode split-vacancy configuration energ:es; (a) unrelaxed, (b) relaxed. All enc gies are expressed in $\mathrm{eV}$.

\begin{tabular}{|c|c|c|}
\hline-7. & (a) Unrelaxed & (b) Relaxed \\
\hline 1.0 & 0.0 & -- \\
\hline 1.10 & 0.126204 & 0.044235 \\
\hline 1.20 & 0.378153 & 0.130203 \\
\hline 1.25 & 0.568115 & 0.098260 \\
\hline 1.30 & 0.803444 & 0.043565 \\
\hline 1.33 & 0.988719 & 0.087490 \\
\hline 1.375 & 1.264458 & 0.118664 \\
\hline 1.50 & 2.259249 & 0.012499 \\
\hline
\end{tabular}

TABLE XI $\perp$

Isotropic Compression: Split-vacancy energies expressed in eV. $E^{\mathrm{x}}(\mathrm{V})$ is the crystal entrgy, $E^{X}(V)_{u r}$ 's the unrelaxed crystal energy, and $E^{r}(V) 1$; the vacancy relaxation energy. $E^{c}(V)$ and $E^{C}(V)$ ur are the vacancy configuration energy ard unrelaxed configuration energy, respectively.

\begin{tabular}{|c|c|c|c|}
\hline$n$ & $\mathrm{E}^{\mathrm{X}}(\mathrm{v}) \mathrm{ur}$ & $E^{x}(v)$ & $E_{r}(V)$ \\
\hline 1.0 & -34.262591 & -34.313054 & 0.050463 \\
\hline 1.15 & -34.648087 & -23.736593 & 0.088506 \\
\hline 1.20 & -33.053327 & -33.171336 & 0.118009 \\
\hline 1.25 & -30.582121 & -30.722715 & 0.140594 \\
\hline 1.30 & -27.126753 & -27.295178 & 0.168425 \\
\hline 1.375 & -19.871463 & -20.088960 & 0.217497 \\
\hline i. 50 & -1.321043 & -1.684286 & 0.362343 \\
\hline$\eta$ & $\underline{I}^{c}(\mathrm{v})_{\mathrm{ur}}$ & $E^{c}(V)$ & $E^{r}(V) / E^{c}(V)$ \\
\hline 1.0 & 0.227477 & 0.177014 & 0.2218 \\
\hline 1.15 & 0.308444 & 0.219938 & 0.2869 \\
\hline 1.20 & 0.337291 & 0.219282 & 0.3499 \\
\hline 1.25 & 0.363812 & 0.223218 & 0.3864 \\
\hline 1.30 & 0.394693 & 0.226268 & 0.4267 \\
\hline 1.375 & 0.443704 & 0.226207 & 0.4902 \\
\hline 1.50 & 0.527935 & 0.165592 & 0.6863 \\
\hline
\end{tabular}


configuration energy. However, the difference in the unrelaxed configuration energies for the SV-IP and SV-OP increases monotonically with uniaxial conpression. The SV-IP and SV-OP configuration energies are negative for $\eta>$ 1.285 and $\eta>1.276$, respectively. A sharp peak occurs in the SV-IP configuration energy at : bout $\eta=1.21$. In contrast, the SV-OP configuration energy rises slightly to a flat peak at $\eta \cong$ 1.10 and thereafter decreases rapidly for $\eta>1.2$.

Tables $X$ and $X I$ show that the SV configuration energy continues to fall with increasing uniaxial compression up to $n=1.5$. Recall that $E^{c}(S C V)$ exhibited a minimum at $\eta=1.365$, as is shown in Fig. 10.

Figure 15 shows that the SV-IP relaxation energy exceeds that for the SV-OP. The datum point at $\eta=\sqrt{2}$ (bcc structure) for the SV-OP stands apart from the wher data points. This feature siggests that additional runs are needed to better define the SV relaxation energy for $1.375 \leqslant n \leqslant 1.5$.

The SV and SCV configuration energies are plotted in Fig. 16 for isotropic compression. $E^{c}(\mathrm{SV})$ rises to a plateau at $\eta=1.15$ and then once more rises slightly to a broad maximum at $\eta=1.33$, and it is positive for all compressions considered. In contrast,

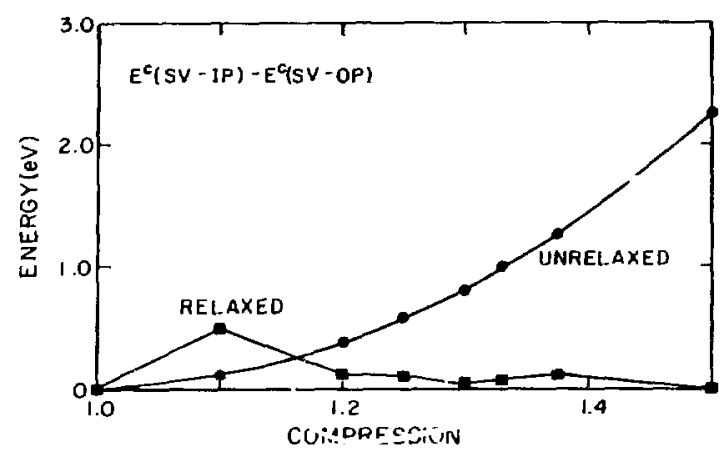

Fig. 14 .

Configuration energy difference for SV$I P$ and SV-OP in the case of uniaxial compression.

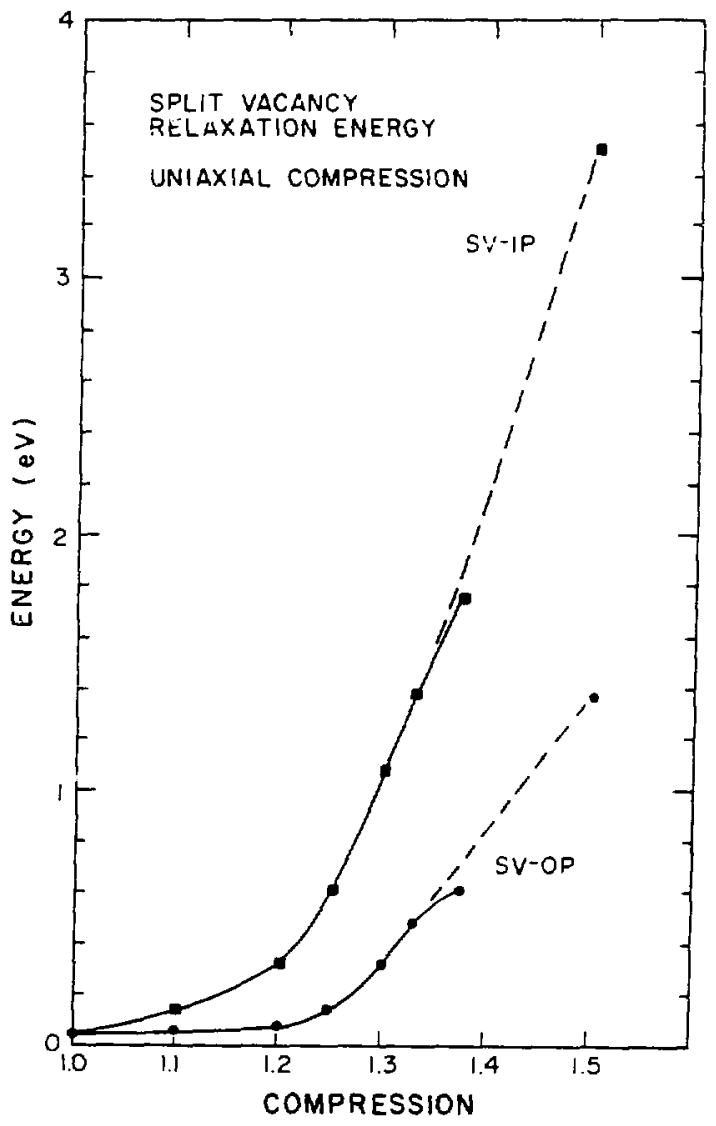

Fig. 15.

Split vacancy relaxation energy for uniaxial compression. 


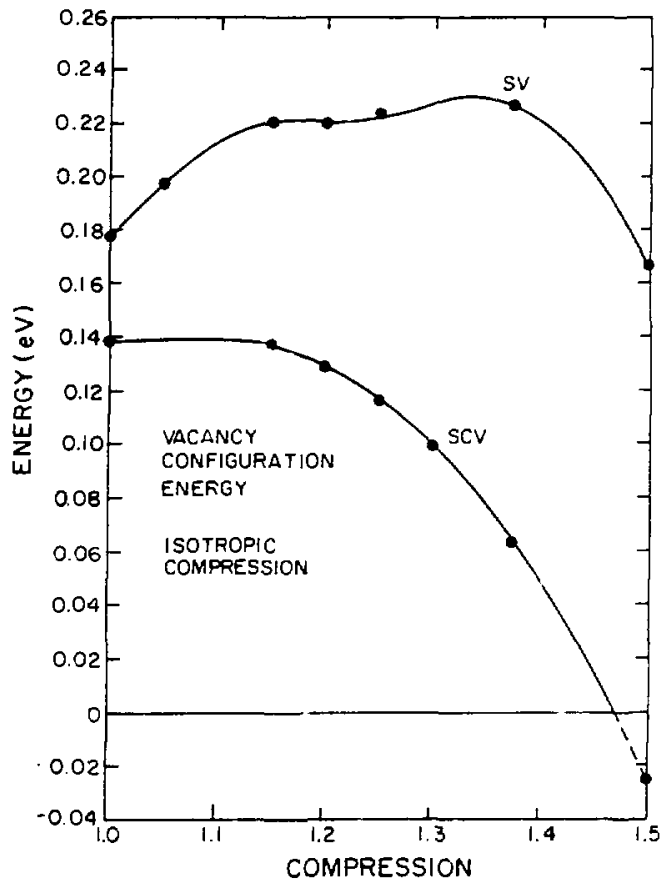

Fig. 16.

SCV and SV configuration energies for isotropic compression.

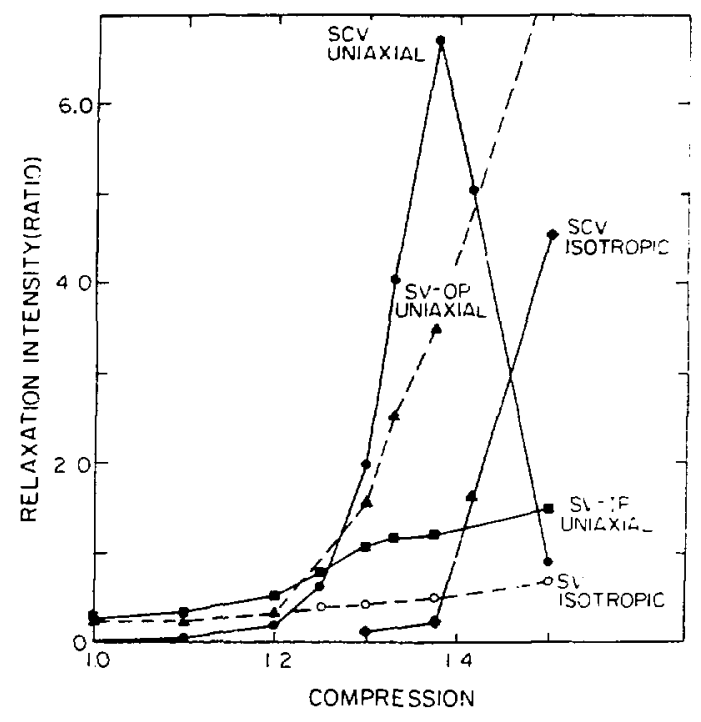

Fig. 17.

Composite plot of SCV and SV relaxation intensities.

$\mathrm{E}^{\mathrm{C}}$ (SCV) decreases with increasing isotropic compression and becomes negative at $\eta=1.47$.

A summary of the relaxation intensity results for vacancies is given in Fig. 17. $I_{r}$ rises to a maximum at $\eta=1.375$ for an SCV during uniaxial compression. In all other cases, $I_{r}$ increases monotonically with compression up to $\eta=1.5$. C. Vacancy Formation Energy

The vacancy formation energy recipe defined in Sec. IV.A is

$$
E^{f}(V)=E^{c}(V)+E^{c l}(P C)
$$

In the case of an SCV, this can be written as

$$
\mathbf{E}^{\mathrm{f}}(\mathrm{SCV})=-\left[\mathrm{E}^{\mathrm{a}}(\mathrm{PC})+\mathrm{E}^{\mathrm{r}}(\mathrm{SCV})\right],
$$

and in the case of an SV, it is 


$$
E^{f}(S V)=E^{c}(S V)_{u r}-E^{r}(S V)+E^{a}(P C)
$$

$E^{f}(S C V)$ and $E^{E}(S V)$ were computed for uniaxial and isotropic compression using the configuration and relaxation energy data given in Tables VII, VIII, X, and XI, together with the $E^{a}(P C)$ data given in Tables IV and $V$. The formation energy results are listed in Tables XIV and XV. The positive formation energies are plotted in Figs. 18 and 19. As indicated by Eq. (14), $\mathrm{E}^{\mathrm{f}}$ (SCV) is dominated

\section{TABLE XIV}

Uniaxial Compression: Vacancy Formation energies expressed in $\mathrm{eV}$.

\begin{tabular}{lrrr}
\multicolumn{1}{r}{$r_{1}$} & $E^{F}(\mathrm{SCV})$ & $\mathrm{E}^{\mathrm{F}}(\mathrm{SV}-\mathrm{IP})$ & $\mathrm{E}^{\mathrm{F}}(\mathrm{SV}-\mathrm{OP})$ \\
\hline 1.0 & 0.0690 & 0.1080 & 0.1080 \\
1.10 & 0.0660 & 0.1568 & 0.1123 \\
1.20 & 0.0423 & 0.2232 & 0.0930 \\
1.25 & -0.0143 & 0.1179 & 0.0196 \\
1.30 & -0.1443 & -0.1186 & -0.1621 \\
1.33 & -0.2971 & -0.2470 & -0.3345 \\
1.375 & -0.3993 & -0.3517 & -0.4704 \\
1.414 & -0.1912 & --- & -0.1231 \\
1.50 & -0.0239 & -1.1938 & -1.2063
\end{tabular}

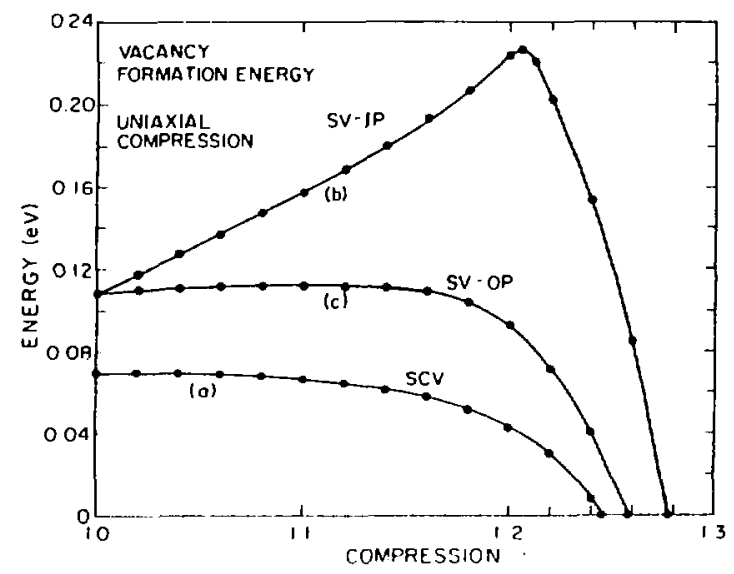

Fig. 18.

SCV, SV-OP, and SV-IP formation energies for uniaxial compression.
TABLE XV

Isotropic Compression: Vacancy formation energies expressed in $\mathrm{eV}$.

\begin{tabular}{|c|c|c|c|}
\hline$n$ & $E^{a}(P C)$ & $E^{F}(S C V)$ & $E^{F}(S V)$ \\
\hline 1.0 & -0.068980 & 0.069007 & 0.108034 \\
\hline 1.10 & -0.069437 & -- & -- \\
\hline 1.15 & -0.069913 & 0.067090 & 0.150025 \\
\hline 1.20 & -0.066781 & 0.062028 & 0.152501 \\
\hline 1.25 & -0.061892 & 0.054036 & 0.161326 \\
\hline 1.30 & -0.055043 & 0.043644 & 0.171225 \\
\hline 1.375 & -0.040630 & 0.022429 & 0.185577 \\
\hline 1.50 & -0.003700 & -0.030096 & 0.161892 \\
\hline
\end{tabular}

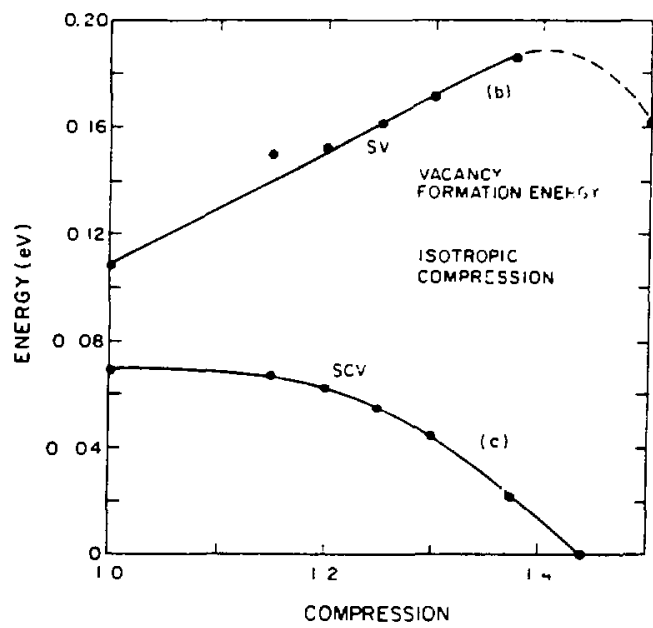

Fig. 19.

SCV and SV formation energies for isotropic compression. 
by $-E^{a}(P C)$ for compression up to 1.2 because $E^{r}$ (SCV) is small for both uniaxial and isotropic compression below 1.2. Consequently, $E^{f}$ (SCV) does not increase with compression because $-E^{a}(P C)$ does not increase with compression. The role of $E^{r}(V)$ always is to reduce the formation energy. The out-of-plane SV formation energy behavior for uniaxial compression is similar to that for an SCV but is larger than the SCV formation energy at a given compression.

Both the uniaxial compression in-flane SV and the isotropic compressicn SV exhibit a larger unrelaxed conifguration energy than do either the SCV or the inplane SV, and these unrelaxed configuration energies increase with compression. Thus, in accordance with Eq. (15), the isotropic compression SV and the uniaxial compression in-plane SV exhibit a formation energy which increases with compression up to $\eta=1.21$ for uniaxial compression and $\eta=1.4$ for isotropic compression.

D. Vacancy Migration Energy

In this discussion, it is assumed that (1) the stable vacancy form in an fct Lennard-Jones crystal is either the SCV, SV-OP, or SV-IP configuration; (2) if the SCV is the stable form, then an SV is the vacancy migration saddle-point configuration; and (3) if an SV is the stable form, then the vacancy migration saddlepoint configuration is either the SCV or the other SV. Assumption (1) virtually is dictated by crystal symmetry. The vacancy migration examples given by Beeler and Beeler (1979) for fcc, bcc, and hcp crystals show that assumptions (2) and should lead to very good estimates of the vacancy migration energy even if the actual saddle-point configurations turned out to be slightly different from those assumed.

Three- and four-point Lagrangian interpolation, applied to the vacancy configuration data in Tables VII, 'III, X, XI, and XIII, was used to prepare more "refined" plots of vacancy configuration energy versus compression than one can make using the direct data given in the tables cited. This was done in order to estimate what particular vacancy form was the stable vacancy form and what particular vacancy form was the vacancy migration saddle point as a function of compression. Table XVI gives the interpolated configuration energy results for uniaxial compression $1.16 \leqslant \eta \leqslant 1.30$. These results are the basis for Fig. 20. Table XVII gives the interpolation results for uniaxial compression $1.30 \leqslant n \leqslant 1.50$. These results are the basis for Fig. 21. Taken together, Figs. 20 and 21 show the following relative ordering among $E^{c}(S C V), E^{c}(S V-O P)$, and $E^{c}(S V-I P)$. 
TABLE XVI

Uniaxial Compression: Interpolated vacancy configuration energies expressed in $\mathrm{eV}$ for $1.16 \leqslant n \leqslant 1.30$.

$\begin{array}{lccc}\eta & E^{C}(S V-O P) & \frac{E^{C}(S V-I P)}{n} & \frac{E^{c}(S C V)}{1} \\ 1.16 & 0.174 & -- & 0.127 \\ 1.18 & 0.168 & -- & 0.119 \\ 1.20 & 0.157 & -- & 0.106 \\ 1.22 & 0.132 & -- & 0.087 \\ 1.24 & 0.099 & --- & 0.061 \\ 1.26 & 0.053 & 0.148 & 0.026 \\ 1.28 & -0.015 & 0.069 & -0.022 \\ 1.30 & -0.113 & -0.071 & -0.095\end{array}$

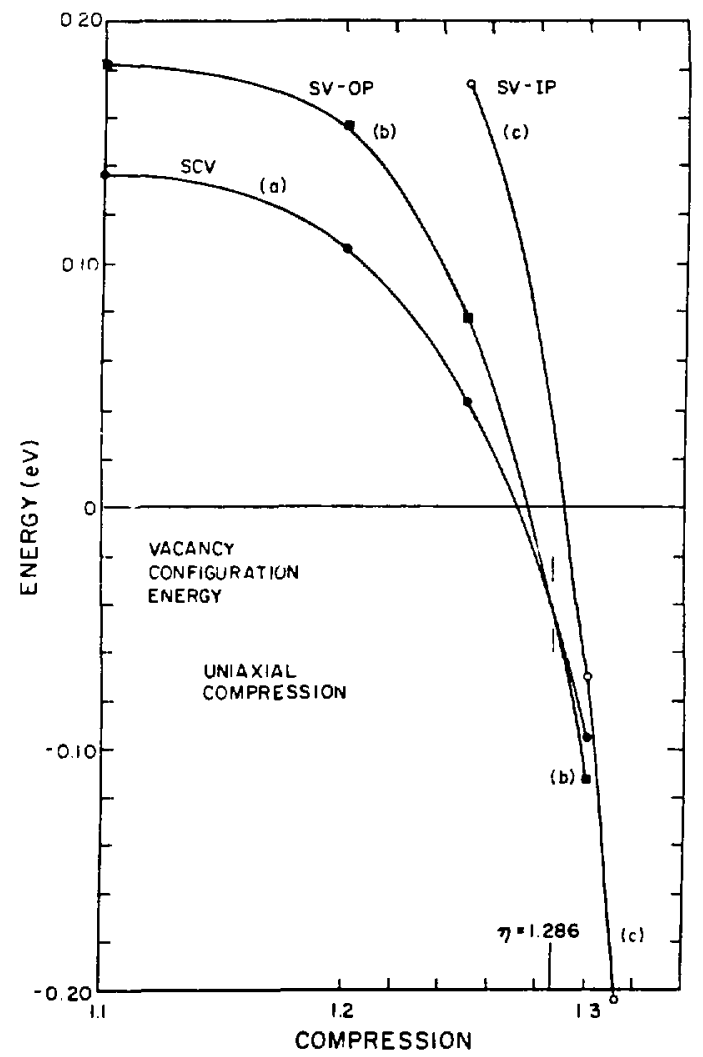

Fig. 20.

SCV, SV-CF, and SV-IP configuration energies in the compression region in which they become negative for uniaxial compression.
TABLE XVII

Uniaxial Compression: Interpolated vacancy configuration energies expressed in $\mathrm{eV}$ for $1.30 \leqslant \eta \leqslant 1.50$.

\begin{tabular}{|c|c|c|c|}
\hline$n$ & $E^{C}(S V-O P)$ & $E^{c}(S V-I P)$ & $E^{c}(S C V)$ \\
\hline 1.3 & -0.113 & -0.071 & -0.095 \\
\hline 1.32 & -0.220 & -0.155 & -0.180 \\
\hline 1.34 & -0.335 & -0.225 & -0.305 \\
\hline 1.36 & -0.402 & -0.275 & -0.375 \\
\hline 1.38 & -0.460 & -0.340 & -0.350 \\
\hline 1.40 & -0.530 & -0.430 & -0.230 \\
\hline 1.42 & -0.625 & -0.535 & -0.150 \\
\hline 1.44 & -0.740 & -0.665 & -0.100 \\
\hline 1.46 & -0.875 & -0.815 & -0.070 \\
\hline 1.48 & -1.07 & -0.995 & -0.045 \\
\hline 1.50 & -1.21 & -1.20 & -0.030 \\
\hline
\end{tabular}

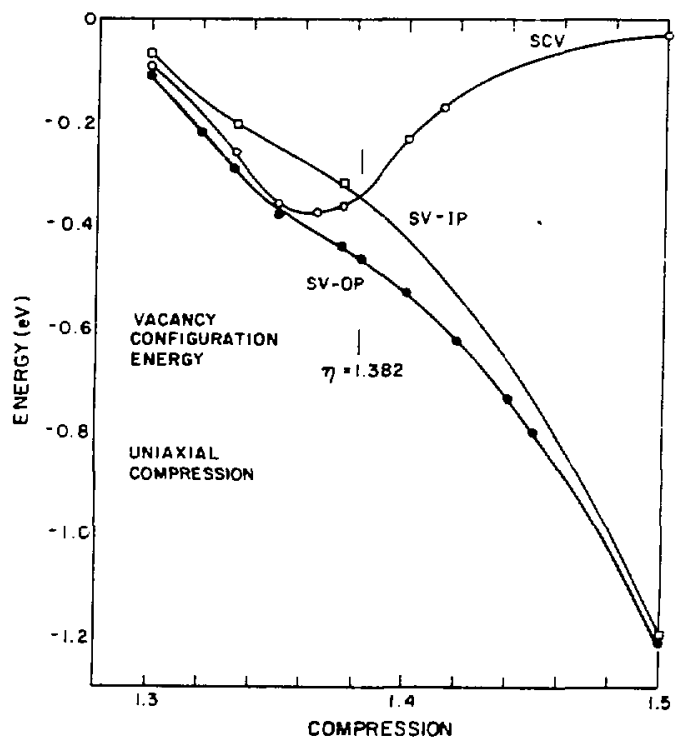

Fig. 21.

Crossing of the SCV and SV-IP configuration energies at $\eta=1.382$. 


$$
\begin{array}{ll}
E^{c}(S C V)<E^{c}(S V-O P)<E^{c}(S V-I P) & 1.0 \leqslant \eta<1.286 \\
E^{c}(S C V)=E^{c}(S V-O P)<E^{c}(S V-I P) & \eta=1.286 \\
E^{c}(S V-O P)<E^{c}(S C V)<E^{c}(S V-I P) & 1.286<\eta<1.382 \\
E^{c}(S C V)=E^{c}(S V-I P) & \eta=1.382 \\
E^{c}(S V-O P)<E^{c}(S V-I P)<E^{c}(S C V) & 1.382<\eta \leqslant 1.50
\end{array}
$$

These relative ordering relations imply the stable vacancy and vacancy migration saddle-point statements given in Table XVIII for uniaxial compression. Table XIX summarizes the vacancy configuration energy data and derived migration energy results for uniaxial compression.

In the casa of isotropic compression, the migration process is straightforward. $E^{C}(S V)>E^{C}(S C V)$ for all compression levels concerned and so the SCV is the stable configuration and the $\mathrm{SV}$ is the vacancy migration saddle point. Table $\mathrm{XX}$ summarizes the vacancy configuration energy data and the derived vacancy mi-

\begin{tabular}{|c|c|c|c|}
\hline $\begin{array}{l}\text { Compression } \\
\text { Mode }\end{array}$ & $\begin{array}{c}\text { Compression } \\
\text { Range }\end{array}$ & $\begin{array}{l}\text { Stable } \\
\text { Vacancy }\end{array}$ & $\begin{array}{c}\text { Vacancy } \\
\text { Migration } \\
\text { Saddle Point } \\
\end{array}$ \\
\hline Isotx & $0.97-1.5$ & $\operatorname{scv}$ & SV \\
\hline Uniaxial & $1.0-1.286$ & $\mathrm{SCV}$ & SV-OP \\
\hline Uniaxial & $1.286-1.382$ & $S V-O P$ & SCV \\
\hline Untaxial & $1.382-1.50$ & $\mathrm{SV}-\mathrm{OP}$ & SV-IP \\
\hline
\end{tabular}
gration energy for isotropic compression.

TABLE XVIII

Stable vacancy and vacancy migration saddle point configurations as a fusction of compression.

TABLE XX

\begin{tabular}{|c|c|c|c|}
\hline$n$ & $E^{c}(S C V)$ & $E^{c}(S V)$ & $E^{m}$ \\
\hline 1.0 & 0.1380 & 0.1770 & 0.0390 \\
\hline 1.15 & 0.1370 & 0.2199 & 0.0829 \\
\hline 1.20 & 0.1288 & 0.2193 & 0.0905 \\
\hline 1.25 & 0.1159 & 0.2232 & 0.1073 \\
\hline 1.30 & 0.0987 & 0.2263 & 0.1276 \\
\hline 1.375 & 0.0631 & 0.2262 & 0.1631 \\
\hline 1.50 & -0.0264 & 0.1656 & 0.1920 \\
\hline
\end{tabular}

Isotropic Compression: Vacancy configuration energies and migration energy expressed in eV as functions of compression.

\begin{tabular}{|c|c|c|c|c|}
\hline$n$ & $E^{c}(S C V)$ & $E^{C}(S V-O P)$ & $\underline{E^{C}(S V-I P)}$ & $E^{m}$ \\
\hline 1.0 & $0.1380^{\mathrm{sc}}$ & $0.1770^{5 P}$ & 0.1770 & 0.0390 \\
\hline 1.1 & $0.1360^{\mathrm{sc}}$ & $0.1826^{5 P}$ & 0.2268 & 0.0466 \\
\hline 1.2 & $0.1062^{\mathrm{sc}}$ & $0.1570^{\mathrm{SP}}$ & 0.2872 & 0.0508 \\
\hline 1.25 & $0.0430^{\mathrm{sc}}$ & $0.0772^{s p}$ & 0.1754 & 0.0342 \\
\hline 1.286 & -0.0400 & -0.0400 & 0.0350 & 0.0 \\
\hline 1.30 & $-0.0953^{5 \mathrm{p}}$ & $-0.1131^{\mathrm{sc}}$ & -0.0696 & 0.0178 \\
\hline 1.33 & $-0.2550^{\mathrm{sp}}$ & $-0.2924^{5 c}$ & -0.2050 & 0.0374 \\
\hline 1.35 & $-0.36^{5 p}$ & $-0.38^{s c}$ & -0.25 & 0.02 \\
\hline 1.375 & $-0.3672^{\mathrm{SP}}$ & $-0.4384^{5 c}$ & -0.3197 & 0.0712 \\
\hline 1.382 & -0.345 & -0.465 & -0.345 & 0.120 \\
\hline 1.40 & -0.23 & $-0.53^{5 c}$ & $-0.43^{S P}$ & 0.10 \\
\hline 1.45 & -0.08 & $-0.80^{\mathrm{sc}}$ & $-0.74^{\mathrm{sp}}$ & 0.06 \\
\hline 1.50 & -0.0322 & $-1.2147^{\mathrm{sc}}$ & $-1.2022^{5 p}$ & 0.0125 \\
\hline
\end{tabular}

\section{TABLE $X I X$}

Uniaxial Compression: Vacancy configuration energies and migration energy expressed in eV as functions of compression. 
The vacancy migration energy is plotted in Fig. 22 for both uniaxial and isotropic compression. The migration energy for isotropic compression exceeds that for uniaxial compression for al1 compressions and exhibits a nearly linear rise with compression. In the case of uniaxial compression, there is a window region, $1.25<n<$ 1.35, within which the vacancy migration energy is less than $0.039 \mathrm{eV}$, the migration energy at $\eta=1.0$. Within the window region the migration energy drops to zero at $\eta=1.286$. A peak of $0.12 \mathrm{eV}$ occurs at $\eta=1.38$, after which

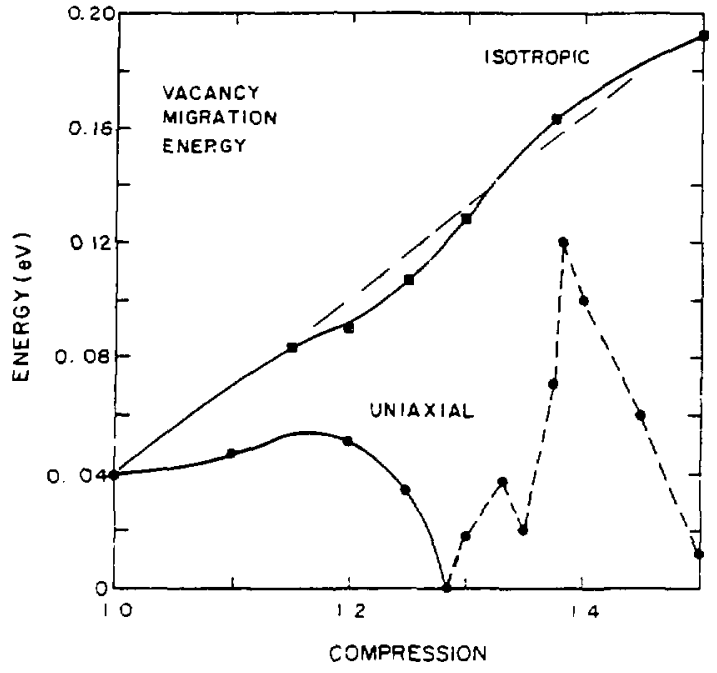

Fig. 22.

Vacancy migration energy vs compression. the migration energy falls almost linearly to $0.0125 \mathrm{eV}$ at $\eta=1.5$. This peak occurs at a compression near that $(\eta=\sqrt{2})$ which gives a bcc structure.

V. VACANCY DISPLACEMENT FIELD IN A COMPRESSED CRYSTAL

This section deals with the displacement field of a vacancy in a compressed crystal. In Sec. IV, we showed that the $\mathrm{SCV}$ was a stable vacancy configuration in each of the isotropic compression runs for $0.94 \leqslant n \leqslant 1.5$. In the case of uniaxial compression, the SCV was the stable vacancy configuration for $\eta<1.29$, but for $1.29<\eta<1.5$, the SV-OP was the stable configuration. The vacancy displacement field pattern for $\eta<1.29$ is qualitatively different from that for $1.29<\eta$ and the nature of this difference illustrates why uniaxial compression changes the form of the stable vacancy, whereas isotropic compression does not. The discussion deals first with the SCV and then the SV-OP.

Vacancy displacement fields provide useful insight concerning the stability of a crystal subjected to uniaxial compression. Because all real crystals contain vacancy defects and edge dislocation line segments, the observations given here on the effect of uniaxial compression on a vacancy displacement field are of fundamental as well as technological interest in the interpretation of shock process data. Edge dislocation line segments are included in this context because the core of an edge dislocation behaves, in part, as a line of vacancies. In 
particular, extended shear displacements observed in a uniaxially compressed crystal containing a line of vacancies should also occur on the tension stiess side of an edge dislocation in a uniaxially compressed crystal. In contrast, vacancy displacement field results for isotropic compression appear to shed little light on shock processes in crystals containing tension stress field defects. A. SCV Displacement Field: Isotropic Compression

The SCV displacement field results for isotropic compression $1.0<\eta \leqslant 1.5$ are qualitatively the same as those for $\eta=1$. This is true because isotropic compression does not alter the crystal symetry. Tables XXI-XXVI describe the SCV displacement field for the first five neighbor shells in the case of isotropic compression.

No atom relaxation occurred about the vacant site for $\eta=1.0$. This was an unexpected result. Because the first simulation was done by creating a vacancy at site 225 in a perfect crystal, it was speculated that perhaps lock-in effects due to perfect crystal symmetry precluded relaxation. Hence, a second simulation was done, in which random initial atom displacements were assigned to break up any possible symmetry lock-in effects. The average displacement was about 0.01 hlc (see Appendix B). However, the result again was no atom relaxation about a va$r$ ancy at $\eta=1.0$. In this regard, Fig. 4b shows that the crystal is subjected to a negative pressure at $\eta=1.0$. We conclude that the null relaxation result for $\eta=1.0$ is a particular consequence of this negative pressure because Table VIII shows that relaxation about an SCV did occur at all other compression levels.

As the compression was increased to 1.5 , significant relaxation occurred only within the first four neighbor shells. At each compression level cited in Tables XXII-XXVI, first-, third-, and fourth-neighhor atoms relaxed inward and secondneighbor atoms relaxed outward from the vacancy. Table XXVI and Fig. 23 express the first-neighbor atom relaxation as a fraction of the interatomic distance at the compression cited. There first-neighbor relaxations ranged from $1.0 \%$ to $1.8 \%$ of the interatomic distance over the compression interval $1.15 \leqslant \eta \leqslant 1.50$.

Figure 24 is a map of the site-centered vacancy displacement field in the $y=4.5$ plane for a vacancy at site 225 and $\eta=1.5$. Sites 173, 175, 2i3, and 275 are first-neighbor sites. Sites 123, 127, 323, and 327 are fourth-neighbor sites. The displacement magnitudes in Fig. 24 are magnified by a factor of five (5X). Second-neighbor atom displacements at sites 125, 223, 227, and 325 are too small to be resolved in Fig. 24 at $5 \mathrm{X}$ magnification. There are no third-neighbor 
Vacancy displacement field for isotropic compression $\eta=1.15$. Site coordinates are expressed in hlc. Atom displacement components are expressed in basal plane hIc.

Site Shell

No. No.

226

227

278

237

228

$\begin{array}{llll}\frac{\mathrm{X}}{n} & & \mathrm{Y} & \mathrm{Z} \\ 5.5 & j .5 & 4.5 \\ 6.5 & 4.5 & 4.5 \\ 6.5 & 5.5 & 5.5 \\ 6.5 & 6.5 & 4.5 \\ 7.5 & 5.5 & 4.5\end{array}$

$\frac{D X}{-0.0100}$
0.0033
-0.0022
-0.0031
0.0004

DY
-0.0100
0.0
-0.0017
-0.0031
0.0

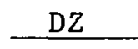

DR

1
2
3
4
5

7.

5.54 .5

0.0004

0.0

0.0

0.0141

TABLE XXII

Vacancy displacement field for isotropic compression $\eta=1.20$. Site coordinates are expressed in hlc. Atom displacement components are expressed in basal plane h1c.

Site Shell

No

\begin{tabular}{|c|c|c|c|c|c|c|c|c|}
\hline No. & No., $n$ & $X$ & $Y$ & Z & DX & DY & $\mathrm{DZ}$ & DR \\
\hline 226 & $I$ & 5.5 & 5.5 & 4.5 & -0.0120 & -0.0120 & 0.0 & 0.0170 \\
\hline 227 & 2 & 6.5 & 4.5 & 4.5 & 0.0030 & 0.0 & 0.0 & 0.0030 \\
\hline 278 & 3 & 6.5 & 5.5 & 5.5 & -0.0028 & -0.0022 & -0.0028 & 0.0045 \\
\hline 237 & 4 & 6.5 & 6.5 & 4.5 & -0.0037 & -0.0037 & 0.0 & 0.0052 \\
\hline 228 & 5 & 7.5 & 5.5 & 4.5 & 0.0005 & 0.0 & 0.0 & 0.0005 \\
\hline
\end{tabular}

\section{TABLE XXIII}

Vacancy displacement field for isotropic compression $\eta=1.25$. Site coordinates are expressed in hlc. Atom displacement components are expressed in basal plane hlc.

Site Shell

No. No., $n$

$226 \quad 1$

X

Y

Z.

DX

DY

$\mathrm{DZ}$

DR

227

5.5

5.5

4.5

$-0.0136$

$-0.0136$

0.0

0.0192

278

6.5

4.5

4.5

0.0030

0.0

0.0

0.0030

237

6.5

5.5

5.5

$-0.0034$

$-0.0024$

$-0.0024$

0.0048

228

$\begin{array}{lll}6.5 & 6.5 \quad 4.5\end{array}$

$-0.0043$

$-0.0043$

0.0

0.0061

$\begin{array}{lll}7.5 & 5.5 & 4.5\end{array}$

$0.0004-0.0002$

0.0

0.0004 
TABLE XXIV

Vacancy displacement field for isotropic compression $\eta=1.30$. Site coordinates are expressed in hlc. Atom displacement components are expressed in basal plane hlc.

\begin{tabular}{|c|c|c|c|c|c|c|c|c|}
\hline $\begin{array}{l}\text { Site } \\
\text { No. }\end{array}$ & $\begin{array}{l}\text { Shell } \\
\text { No., } n\end{array}$ & $\underline{X}$ & $Y$ & Z & $\mathrm{DX}$ & $D_{2}^{r y}$ & DZ & $\mathrm{DR}$ \\
\hline 226 & 1 & 5.5 & 5.5 & 4.5 & -0.0147 & -0.0147 & 0.0 & 0.0208 \\
\hline 227 & 2 & 6.5 & 4.5 & 4.5 & 0.0026 & 0.0 & 0.0 & 0.0026 \\
\hline 278 & 3 & 6.5 & 5.5 & 5.5 & -0.0039 & -0.0028 & -0.0028 & 0.0056 \\
\hline 237 & 4 & 6.5 & 6.5 & 4.5 & -0.0048 & -0.0048 & 0.0 & 0.0068 \\
\hline 228 & 5 & 7.5 & 5.5 & 4.5 & 0.0003 & -0.0002 & 0.0 & 0.0004 \\
\hline
\end{tabular}

TABLE XXV

Vacancy displacement field for isotropic compression $\eta=1.375$. Site coordinates are expressed in hlc. Atom displacement components are expressed in basil plane hlc.

\begin{tabular}{|c|c|c|c|c|c|c|c|c|}
\hline $\begin{array}{l}\text { Site } \\
\text { No. }\end{array}$ & $\begin{array}{l}\text { She1l } \\
\text { No., n } \\
\end{array}$ & $\underline{X}$ & $\mathrm{Y}$ & 2 & $\mathrm{DX}$ & DY & DZ & DR \\
\hline 226 & 1 & 5.5 & 5.5 & 4.5 & -0.0162 & -0.0162 & 0.0 & 0.0229 \\
\hline 227 & 2 & 6.5 & 4.5 & 4.5 & 0.0024 & 0.0 & 0.0 & 0.0024 \\
\hline 278 & 3 & 6.5 & 5.5 & 5.5 & -0.0044 & -0.0032 & -0.0032 & 0.0063 \\
\hline 237 & 4 & 6.5 & 6.5 & 4.5 & -0.0054 & -0.0054 & 0.0 & $0.007 \epsilon$ \\
\hline 228 & 5 & 7.5 & 5.5 & 4.5 & 0.0002 & 0.0002 & 0.0 & 0.0002 \\
\hline
\end{tabular}

TABLE XXVI

Vacancy displacement field for isotropic compression $\eta=1.50$. Site coordinates are expressed in hlc. Atom displacement components are expressed in basal plane hlc.

Site Shel1

No. No., n

$226 \quad 1$

X $\quad$ Y $\quad \underline{Z}$

$5.5 \quad 5.5$

4.5

$\frac{\text { DX }}{-0.0179}$

DY

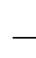

DZ

DR

$227 \quad 2$

$6.5 \quad 4.5$

4.5

0.0022

$-0.0179$

0.0

0.0252

278

6.5

5.5

5.5

$-0.0049$

0.0

0.0

0.0022

237

6.5

6.5

4.5

$-0.0059$

$-0.0036$

$-0.0036$

0.0062

228

7.5

5.5

4.5

0.0002

$-0.0059$

0.0

0.0083

5

7.5

$-0.0004$

0.0

0.0004 
atom sites in the $y=4.5$ plane. The coordinates in Fig. 24 are expressed in half-lattjec-constant (hlc) units at a compression of 1.5 .

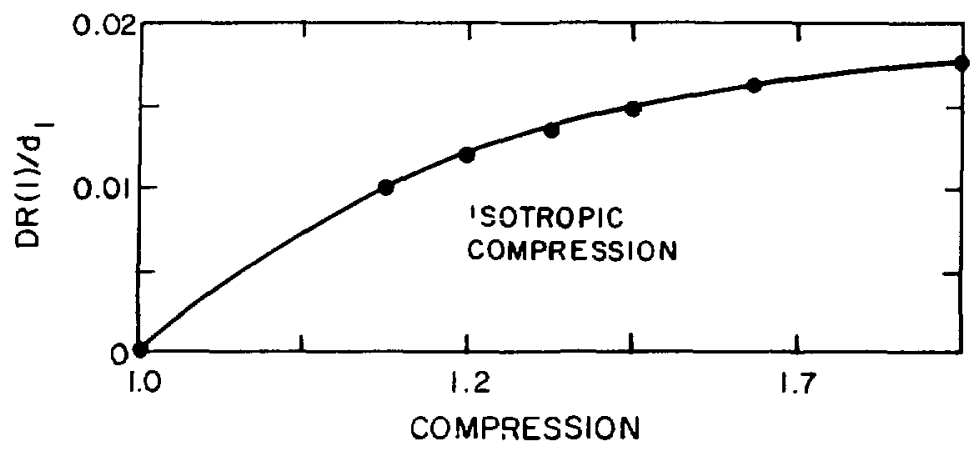

Fig. 23.

First-neighbor radial displacement DR(I) expressed as a Eraction of the basal plane interatomic distance $\mathrm{d}_{1}$ for an SCV and isotropic compression.

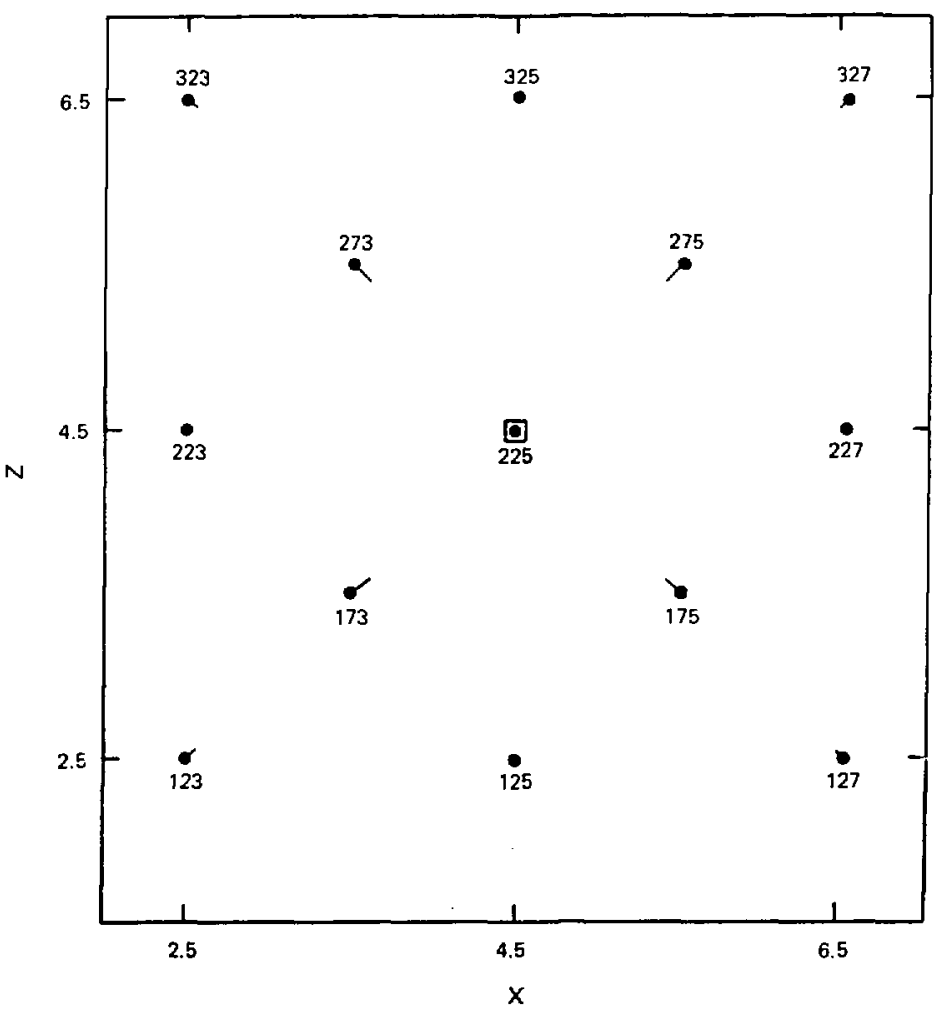

Fig. 24.

SCV displacement field map for isotropic compres sion equal to 1.50 . The $y=4.5$ plane shown here passes through the vacancy at site 225. The displacement component DY is zero for all atoms in the $y=4.5$ plane. The radius of each circle used to denote site positions is equal to $1 \%$ of the basal plane interatomic distance at a magnification $₹ 5 \mathrm{X}$. The length of the 1 -ial line drawn from the center of a circle is the radial displacement vector at magnification $5 \mathrm{X}$. 
B. Site-Centered Vacancy Displacement Field: Uniaxial Compression

Figures 25-35 are plots of atom relaxation about a site-centered vacancy at site 225 versus unfaxial compression along [001], for each of the first ten neighbor shells. The neighbor shell designation cited pertains to the location of the atom noted in an $\mathrm{fcc}$ crystal when $\eta=1.0$. These figures show that displacement magnitudes for the first-, second-, and third-neighbor shells tend to increase as compression increases up to $\eta=1.3$ and then tend to decrease as compression increases from 1.3 to 1.5 . Displacement magnitudes in the fourth- and tenthneighbor shells increase with increasing compression up to $\eta=1.375$, and then

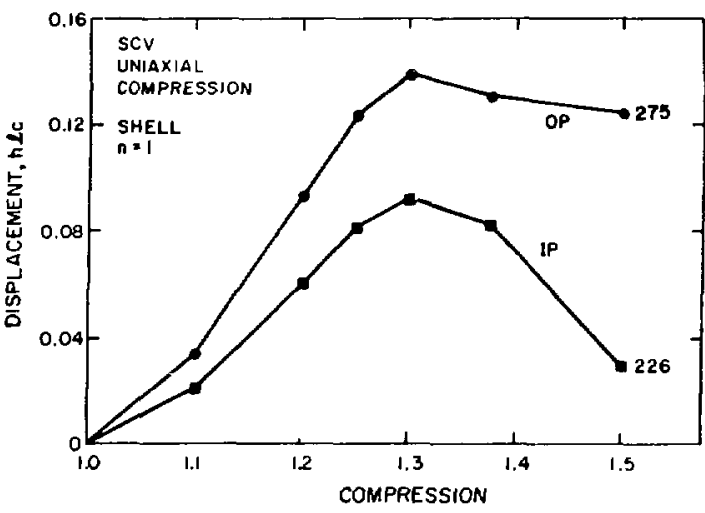

Fig. 25.

Radial displacement in basal plane halflattice constants for in-plane and outof-plane first neighbors of an SCV vs uniaxial compression.

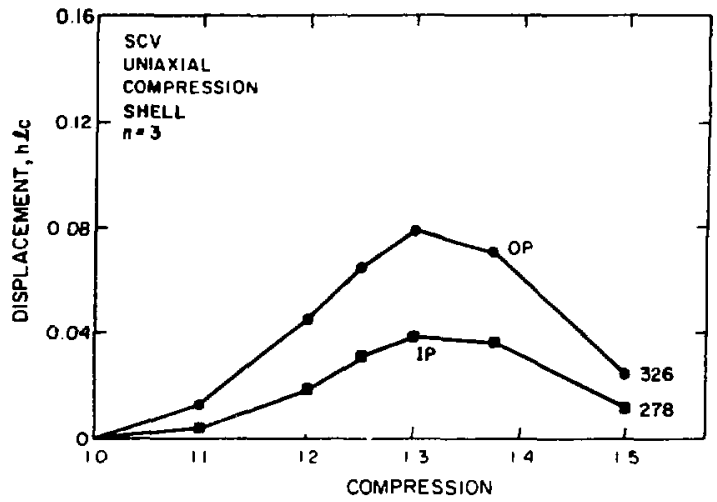

Fig. 27 .

Radial displacement for third neighbors of an SCV vs uniaxial compression.

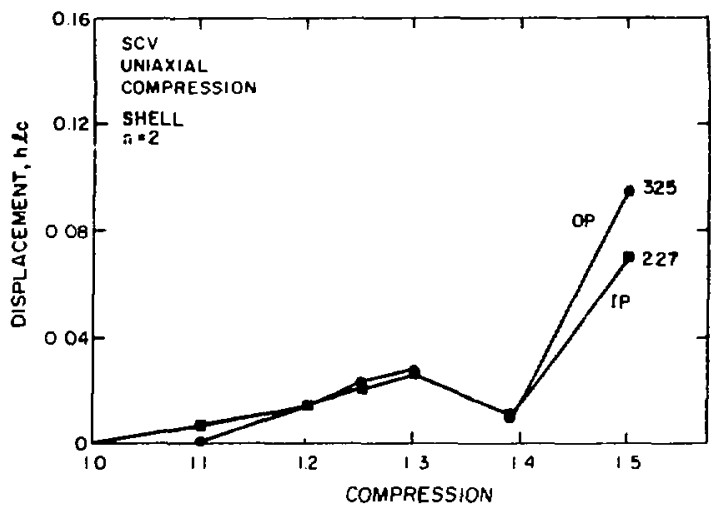

Fig. 26.

Radial displacement for second neighbors of an SCV vs uniaxial compression.

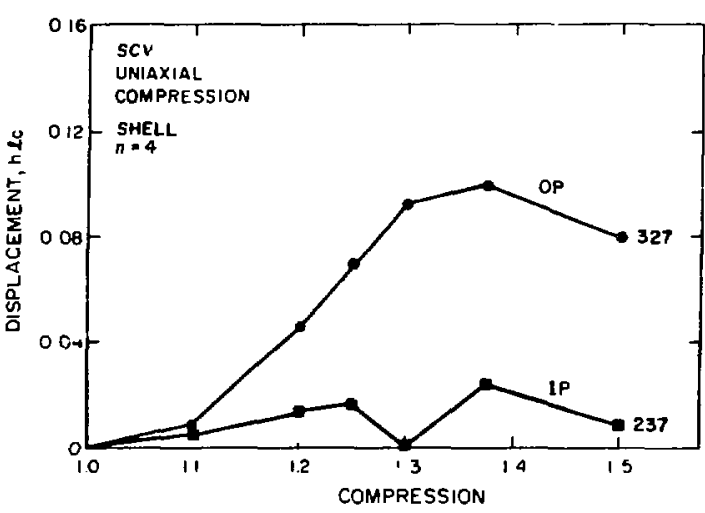

Fig. 28.

Radial displacement for fourth neighbors of an SCV vs uniaxial compression. 


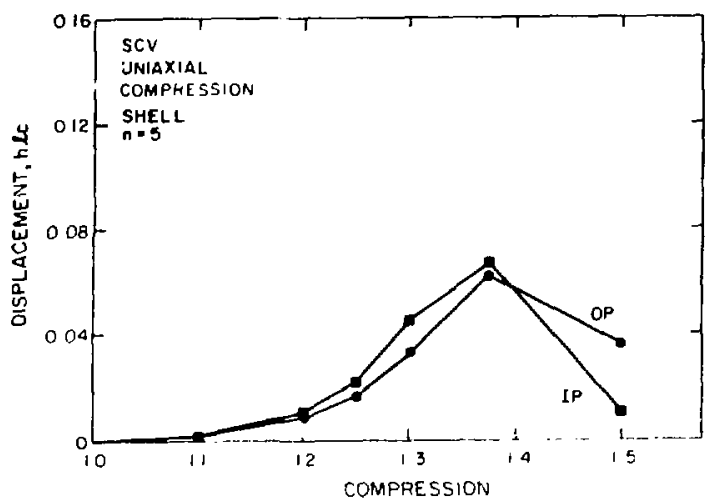

Fig. 29.

Radial displacement for fifth neighbors of an SCV vs uniaxial compression.

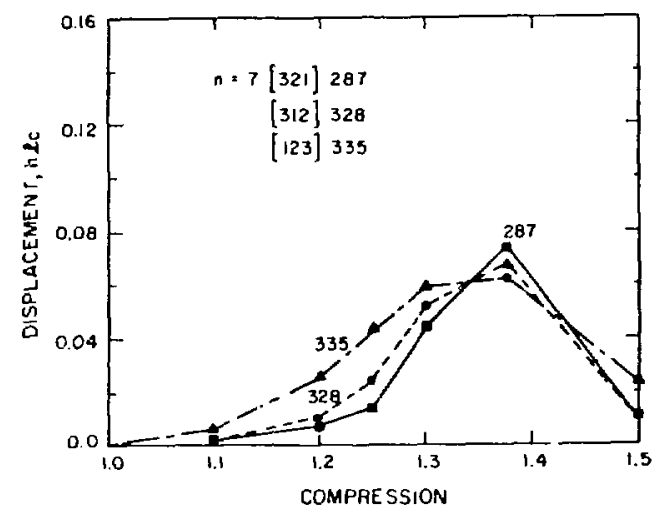

Fig. 31 .

Radial displacement for seventh neighbors of an SCV vs uniaxial compression.

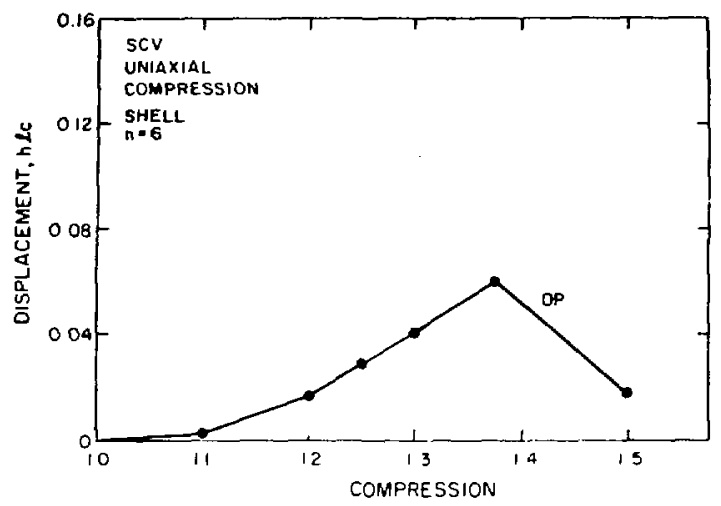

Fig. 30 .

Radial displacement for sixth neighbors of an SCV vs uniaxial compression.

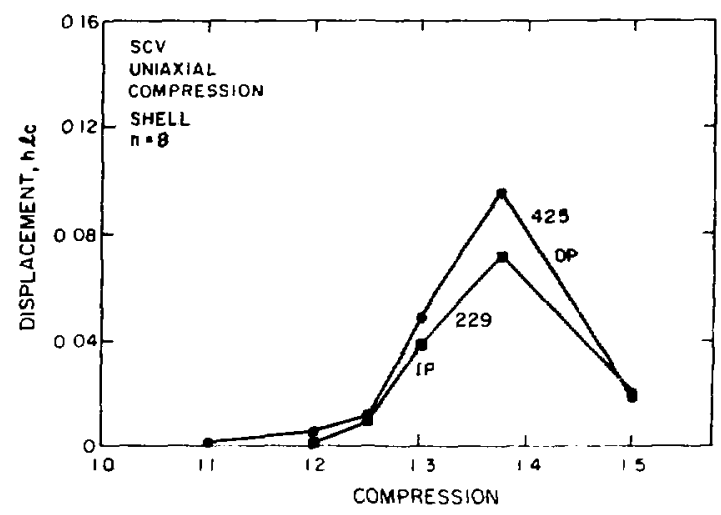

Fig. 32 .

Radial displacement for eighth neighbors of an SCV vs uniaxial compression.

decrease as compression increases from 1.375 to 1.50 . In most instances, the displacement magnitudes for the out-of-plane subshells are greater than those for the in-plane subshells. A description of in-plane and out-of-plane neighbor subshells is given in Appendix $C$.

In short, out-of-plane displacements about the vacancy increase with uniaxial compression up to a certain compression level as the result of augmented displacement components parallel to the uniaxial compression stress axis. However, this effect eventually is thwarted when the increase in atom number density, due to increasing compression, begins to restrict tho range of atom movement. In 


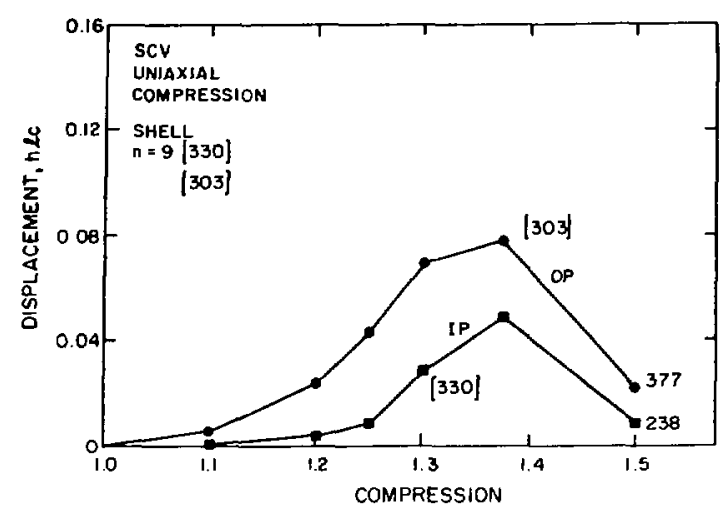

Fig. 33.

Radial displacement for [330] and [303] ninth neighbors of an SCV vs uniaxial compression.

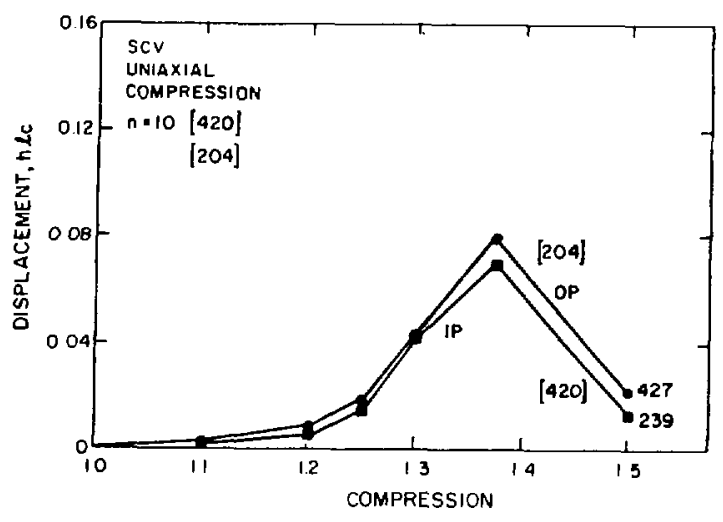

Fig. 35.

Radial displacement for [420] and [204] tenth neighbors of an SCV vs uniaxial compression.

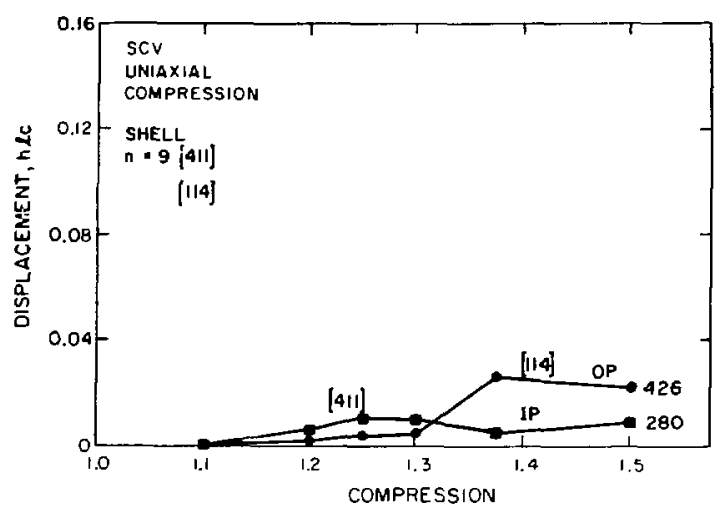

Fig. 34.

Radial displacement for [411] and [114] ninth neighbors of an SCV vs uniaxial compression.

other words, increasing compression leads to a crowding effect which tends to reduce individuai a .om displacement magnitudes even though the repulsive force atom pair interaction increases monotonically with compression. The attenuating effect of crowding on firstneighbor atom relaxation about a vacan$c y$, in the case of isotropic compression, is illustrated in Fig. 23. This figure suggests a saturation effect for isotropic compression.

$$
\text { Displacement field maps are given }
$$

in Figs. 36-42 for uniaxial compression.

Each of these maps describes the displacement of atoms in the $y=4.5$ plane through a site-centered vacancy at site 225. At $\eta=1.10$ (Fig. 36), the displacement map resembles that for isotropic compression $\eta=1.50$ (see Fig. 24) in that only the relaxation of first-neighbor atoms is significant. As compression increases, one notices that the number of atom sites at which the atom displacement exceeds $1 \%$ of the interatomic distance (measured at $\eta=1.0$ ) also increases. Atom displacements are listed in Tables XXVII-XXXIII. 


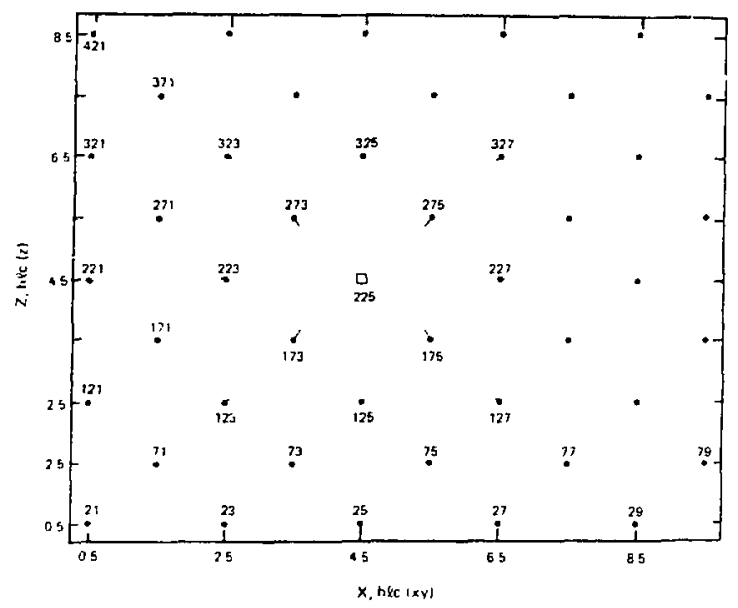

Fig. 36.

Displacement field map for the $y=4.5$ plane through an SCV for uniaxial compression equal to 1.10 . The radius of each circle used to denote the position of an atom site is equal to $1 \%$ of the basal plane interatomic distance at a magnification of $5 \mathrm{X}$. The radial line marked for a site is the radial atom displacement at that site at magnification 5X. This notation is used in each succeeding displacement field map.

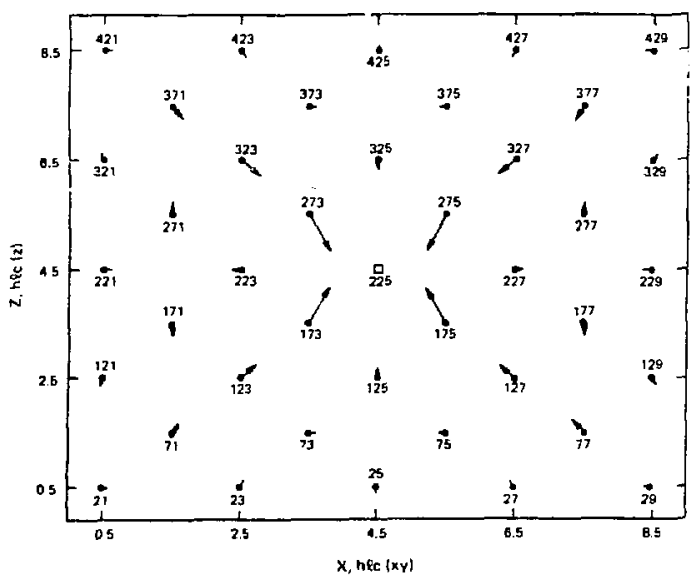

Fig. 38 .

Displacement field map for the $y=4.5$ plane through an SCV for lidiaxial compression equal to 1.25 .

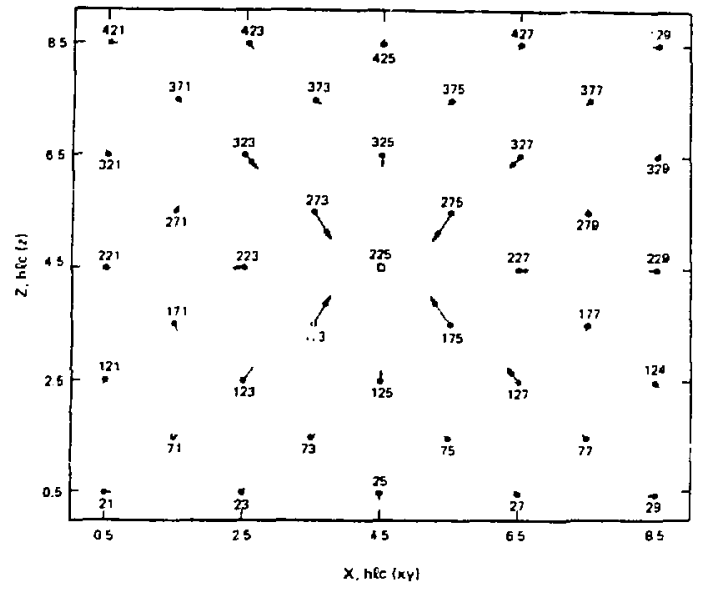

Fig. 37 .

Displacement field map for the $y=4.5$ plane thrcugh an SCV for uniaxial compression equal to 1.20 .

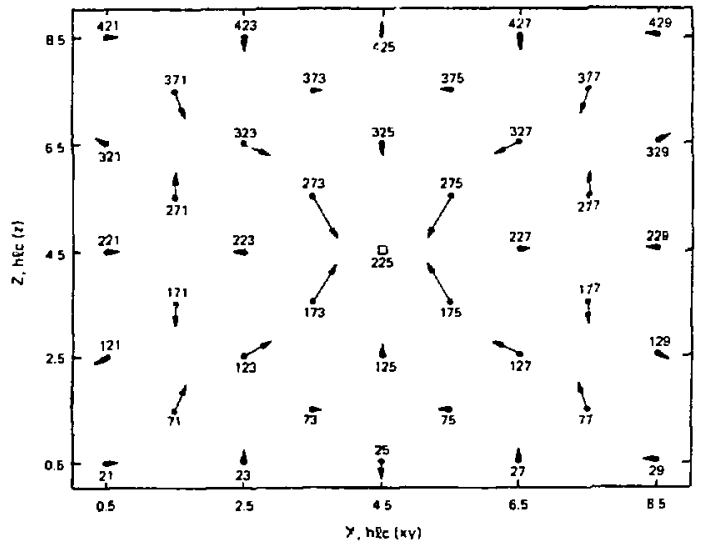

Fig. 39.

Displacement field map for the $y=4.5$ plane through an SCV for uniaxial compression equal to 1.30 . 


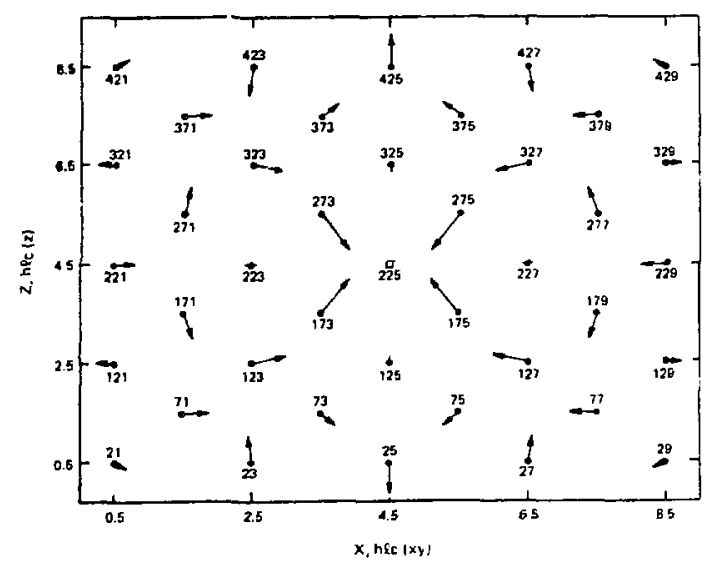

Fig. 40.

Displacement field map for the $y=4.5$ plane through an SCV for uniaxial compression equal to 1.375 .

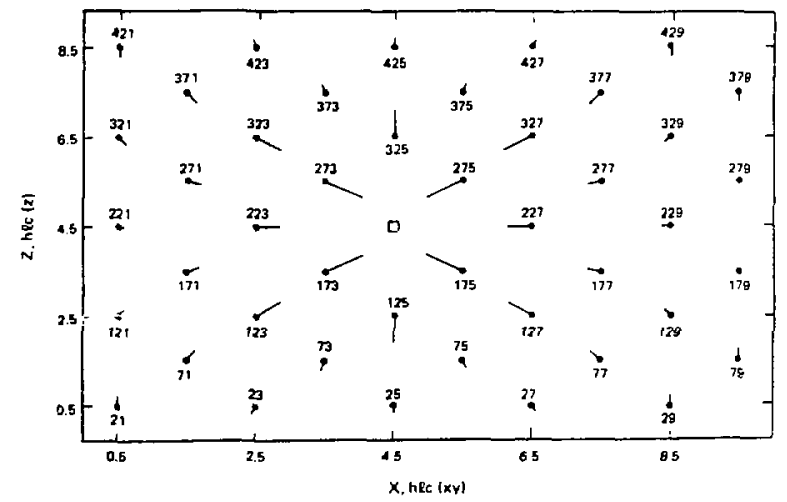

Fig. 42.

Displacement field map for the $y=4.5$ plane through an SCV for uniaxial compression equal to 1.50 .

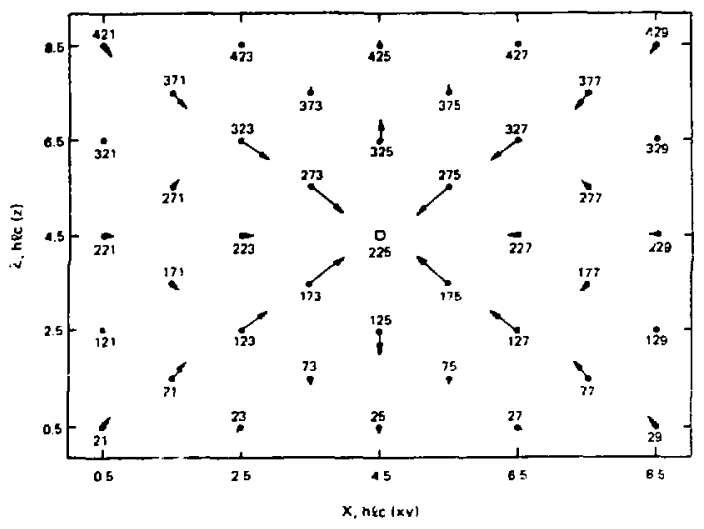

Fig. 41.

Displacement field map for the $y=4.5$ plane through an SCV for uniaxial compression equal to 1.414 .

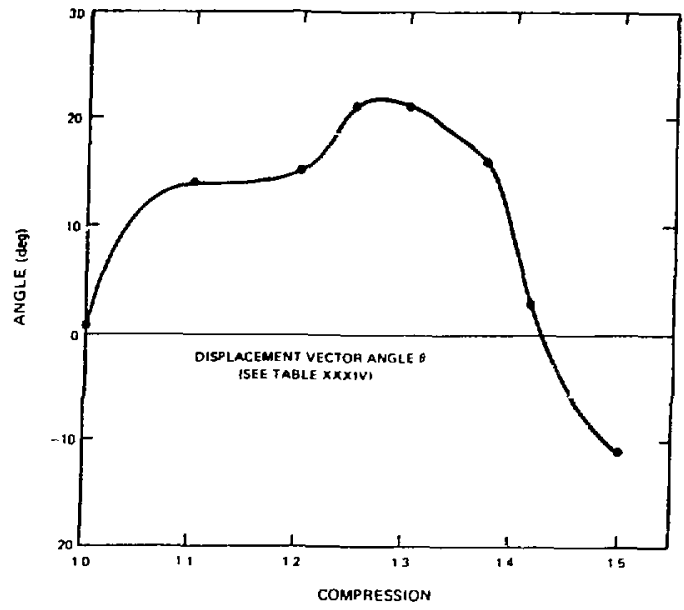

Fig. 43.

First-neighbor displacement vector angle for an SCV.

Figure 43 and Table XXXIV describe the variation of the angle $\theta$ between the out-of-plane first-neighbor displacement vector for the atom at site 173 and the line from site 173 to the vacant site at site 225, as $\eta$ incroases from 1.0 to 1.5. $\theta$ is positive and increases with increasing $\eta$ up to $\eta$ 1.275. This means that the displacement vector deviates progressively from the site-to-site line direction toward the z-axis. $\theta$ then decreases from a maximum of $22^{\circ}$ to zero as $\eta$ increases from 1.275 to 1.425 . Thereafter, $\theta$ becomes progressively more negative 
Uniaxial Compression $\eta=1.10: \quad 3 C V$ displacement field (DX, DY, DZ) in the $y=$ 4.5 plane through the vacancy at site 225. DY $=0$ for all sites in the $y=4.5$ plane. DK, DY, and DR are expressed in hlc (xy).

\begin{tabular}{|c|c|c|c|c|c|c|c|}
\hline Site & DX & $D Z$ & DR & Site & $\mathrm{DX}$ & $\mathrm{DZ}$ & $\mathrm{DR}$ \\
\hline 471 & 0.0016 & 0.0 & 0.0016 & 221 & -0.0006 & 0.0 & 0.0006 \\
\hline 473 & 0.0003 & 0.0 & 0.0003 & 223 & -0.0069 & 0.0 & 0.0069 \\
\hline 475 & -0.0003 & 0.0 & 0.0003 & 225 & 0.0 & 0.0 & 0.0 \\
\hline 477 & -0.0017 & 0.0 & 0.0017 & 227 & 0.0068 & 0.0 & 0.0068 \\
\hline 479 & 0.0 & 0.0 & 0.0 & 229 & 0.0007 & 0.0 & 0.0007 \\
\hline 421 & 0.0022 & -0.0009 & 0.0024 & 171 & -0.0022 & -0.0014 & 0.0026 \\
\hline 423 & 0.0013 & -0.0009 & 0.0016 & 173 & 0.0191 & 0.0283 & 0.0341 \\
\hline 425 & 0.0 & 0.0 & 0.0 & 175 & -0.0189 & 0.0283 & 0.0340 \\
\hline 427 & -0.0013 & -0.0009 & 0.0016 & 177 & 0.0023 & -0.0014 & 0.0027 \\
\hline 429 & -0.0023 & -0.0009 & 0.0025 & 179 & 0.0 & -0.0005 & 0.0005 \\
\hline 371 & 0.0043 & -0.0036 & 0.0056 & 121 & -0.0008 & -0.0012 & 0.0014 \\
\hline 373 & 0.0010 & -0.0011 & 0.0015 & 123 & 0.0092 & 0.0092 & 0.0130 \\
\hline 375 & -0.0011 & -0.0011 & 0.0016 & 125 & 0.0 & 0.0005 & 0.0005 \\
\hline 377 & -0.0044 & 0.0036 & 0.0057 & 127 & -0.0092 & 0.0092 & 0.0130 \\
\hline 379 & 0.0 & 0.0013 & 0.0013 & 129 & 0.0007 & -0.0012 & 0.0014 \\
\hline 321 & -0.0008 & 0.0012 & 0.0014 & 71 & 0.0043 & 0.0036 & 0.0056 \\
\hline 323 & 0.0092 & -0.0093 & 0.0131 & 73 & 0.0010 & 0.0011 & 0.0015 \\
\hline 325 & 0.0 & -0.0006 & 0.0006 & 75 & -0.0011 & 0.0011 & 0.0016 \\
\hline 327 & -0.0092 & -0.0093 & 0.0131 & 77 & -0.0044 & 0.0036 & 0.0057 \\
\hline 329 & 0.0067 & 0.0012 & 0.0014 & 79 & 0.0 & -0.0013 & 0.0013 \\
\hline 271 & -0.0022 & 0.0014 & 0.0026 & 21 & 0.0022 & 0.0008 & 0.0023 \\
\hline 273 & 0.0188 & -0.0282 & 0.0339 & 23 & 0.0013 & 0.0008 & 0.0015 \\
\hline 275 & -0.0189 & -0.0282 & 0.0339 & 25 & 0.0 & 0.0 & 0.0 \\
\hline 277 & 0.0023 & 0.0014 & 0.0027 & 27 & -0.0013 & 0.0008 & 0.0015 \\
\hline 279 & 0.0 & 0.0005 & 0.0005 & 29 & -0.0023 & 0.0008 & 0.0024 \\
\hline
\end{tabular}

as $\eta$ increases to 1.5 . This means that for $\eta>1.425$, the disnlacement vector deviates progressively from the site-to-site line toward the $x$-axis. In a qualitative sense, uriaxial stress first tends to rotate the atom displacement vector away from the close-packed line direction toward the stress axis $F_{i} \eta<1.275$. This effect persists with diminishing strength up to $\eta=1.425$. Thereafter, an increase in axial stress inhibits displacement along the stress axis and produces a relatively greater displacement component normal to the stress axis. The behavior described by Table XXXIV and Fig. 43 is critical behavior in that for $1.275<\eta<1.425$ the crystal is in a transition region, due to the 
TABLE XXVIII

Uniaxial Compression $\eta=1.20$ : SCV displacement $\mathrm{field}$ ( $\mathrm{DX}, \mathrm{DY}, \mathrm{DZ}$ ) in the $\mathrm{y}=$ 4.5 plane through the vacancy at site 225. DY $=0$ for all sites in the $y=4.5$ plane. DX, DY, and DR are expressed in hlc (xy).

\begin{tabular}{|c|c|c|c|c|c|c|c|}
\hline Site & $\mathrm{DX}$ & $\mathrm{DZ}$ & $\mathrm{DR}$ & Site & $\mathrm{DX}$ & $\mathrm{DZ}$ & $\mathrm{DR}$ \\
\hline 471 & 0.0094 & 0.0 & 0.0 & 221 & 0.0013 & 0.0 & 0.0013 \\
\hline 473 & -0.0014 & 0.0 & 0.0014 & 223 & -0.0151 & 0.0 & 0.0151 \\
\hline 475 & 0.0013 & 0.0 & 0.0012 & 225 & 0.0 & 0.0 & 0.0 \\
\hline 477 & -0.0095 & 0.0 & 0.0095 & 227 & 0.0151 & 0.0 & 0.0151 \\
\hline 479 & -0.0082 & 0.0 & 0.0082 & 229 & -0.0013 & 0.0 & 0.0013 \\
\hline 421 & 0.0118 & -0.0029 & 0.0122 & 171 & -0.0043 & -0.0098 & 0.0107 \\
\hline 423 & 0.0057 & -0.0056 & 0.0080 & 173 & 0.0474 & 0.0796 & 0.0927 \\
\hline 425 & 0.0 & 0.0050 & 0.0050 & 175 & -0.0474 & 0.0796 & 0.0927 \\
\hline 427 & -0.0058 & -0.0056 & 0.0080 & 177 & 0.0042 & -0.0112 & 0.0119 \\
\hline 429 & -0.0117 & -0.0029 & 0.0121 & 179 & 0.0 & -0.0013 & 0.0013 \\
\hline 371 & 0.0177 & -0.0168 & 0.0245 & 121 & -0.0042 & -0.0083 & 0.0092 \\
\hline 373 & 0.0081 & -0.0046 & 0.0093 & 123 & 0.0337 & 0.0308 & 0.0456 \\
\hline 375 & -0.0080 & -0.0046 & 0.0092 & 125 & 0.0 & 0.0144 & 0.0144 \\
\hline 377 & -0.0177 & -0.0168 & 0.0245 & 127 & -0.0336 & 0.0307 & 0.0456 \\
\hline 379 & 0.0 & 0.0114 & 0.0114 & 129 & 0.0042 & -0.0082 & 0.0093 \\
\hline 321 & -0.0042 & 0.0083 & 0.0092 & 71 & 0.0177 & 0.0168 & 0.0245 \\
\hline 323 & -0.0337 & -0.0308 & 0.0456 & 73 & 0.0081 & 0.0045 & 0.0093 \\
\hline 325 & 0.0 & -0.0144 & 0.0144 & 75 & -0.0080 & 0.0045 & 0.0092 \\
\hline 327 & -0.0336 & -0.0308 & 0.0456 & 77 & -0.0177 & 0.0168 & 0.0245 \\
\hline 329 & 0.0042 & 0.0083 & 0.0093 & 79 & 0.0 & -0.0114 & 0.0114 \\
\hline 271 & -0.0043 & 0.0112 & 0.0120 & 21 & 0.0118 & 0.0029 & 0.0122 \\
\hline 273 & 0.0474 & -0.0796 & 0.0927 & 23 & 0.0057 & 0.0656 & 0.0080 \\
\hline 275 & -0.0474 & -0.0796 & 0.0927 & 25 & 0.0 & -0.0050 & 0.0050 \\
\hline 277 & 0.0042 & 0.0134 & 0.0140 & 27 & -0.0058 & 0.0056 & 0.0081 \\
\hline 279 & $0 . n$ & 0.0013 & 0.0013 & 29 & -0.0117 & 0.0029 & 0.0121 \\
\hline
\end{tabular}

crowding effect, wherein increased uniaxial compression tends to produce a larger transverse atom displacement, away from the vacancy, than an axial displacement toward the vacancy. This effect is the root from which grow extended shear displacement fields about either an SCV or an SV-OP for $\eta>1.29$. In an attempt to relieve axial crowding, the crystal turns to transverse crowding. 
Uniaxial Compression $\eta=1.25$ : SCV displacement field (DX, DY, DZ) in the $y=$ 4.5 plane through the vacancy at site 225 . DY $=0$ for all sites in the $y=4.5$ plane. DX, DY, and DR are expressed in hlc (xy).

\begin{tabular}{|c|c|c|c|c|c|c|c|}
\hline Site & DX & $\mathrm{DZ}$ & DR & Site & DX & $\mathrm{DZ}$ & DR \\
\hline 471 & 0.0203 & 0.0 & 0.0203 & 221 & 0.0092 & 0.0 & 0.0092 \\
\hline 473 & -0.0053 & 0.0 & 0.0053 & 223 & -0.0212 & 0.0 & 0.0212 \\
\hline 475 & 0.0054 & 0.0 & 0.0054 & 225 & 0.0 & 0.0 & 0.0 \\
\hline 477 & -0.0204 & 0.0 & 0.0204 & 227 & 0.0212 & 0.0 & 0.0212 \\
\hline 479 & 0.0 & 0.0 & 0.0 & 229 & -0.0092 & 0.0 & 0.0092 \\
\hline 421 & 0.0234 & -0.0022 & 0.0235 & 171 & -0.0043 & -0.0281 & 0.0285 \\
\hline 423 & 0.0092 & -0.0149 & 0.0175 & 173 & 0.0620 & 0.1058 & 0.1226 \\
\hline 425 & 0.0 & 0.0114 & 0.0114 & 175 & -0.0619 & 0.1058 & 0.1226 \\
\hline 427 & -0.0092 & 0.0149 & 0.0175 & 177 & 0.0042 & -0.0281 & 0.0284 \\
\hline 429 & -0.0233 & -0.0022 & 0.0234 & 179 & 0.0 & 0.0050 & 0.0050 \\
\hline 371 & 0.0274 & -0.0326 & 0.0425 & 121 & -0.0121 & -0.0171 & 0.0210 \\
\hline 373 & 0.0153 & -0.0067 & 0.0167 & 123 & 0.0535 & 0.0445 & 0.0696 \\
\hline 375 & -0.0154 & -0.0067 & 0.0168 & 125 & 0.0 & 0.0234 & 0.0234 \\
\hline 377 & -0.0273 & -0.0326 & 0.0425 & 127 & -0.0534 & 0.0445 & 0.0696 \\
\hline 379 & 0.0 & 0.0309 & 0.0309 & 129 & 0.0122 & -0.0171 & 0.0210 \\
\hline 321 & -0.0121 & 0.0172 & 0.0210 & 71 & 0.0274 & 0.0326 & 0.0426 \\
\hline 323 & 0.0535 & -0.0445 & 0.0696 & 73 & 0.0153 & 0.0067 & 0.0167 \\
\hline 325 & 0.0 & -0.0236 & 0.0236 & 75 & -0.0154 & 0.0067 & 0.0168 \\
\hline 327 & -0.0534 & -0.0445 & 0.0695 & 77 & -0.0273 & 0.0326 & 0.0425 \\
\hline 329 & 0.0122 & 0.0172 & 0.0210 & 79 & 0.0 & -0.0310 & 0.0310 \\
\hline 271 & -0.0043 & 0.0282 & 0.0285 & 21 & 0.0234 & 0.0021 & 0.0235 \\
\hline 273 & 0.0620 & -0.1059 & 0.1227 & 23 & 0.0092 & 0.0148 & 0.0174 \\
\hline 275 & -0.0619 & -0.1059 & 0.1227 & 25 & 0.0 & -0.0114 & 0.0114 \\
\hline 277 & 0.0042 & 0.0282 & 0.0285 & 27 & -0.0092 & 0.0148 & 0.0174 \\
\hline 279 & 0.0 & -0.0049 & 0.0049 & 29 & -0.0233 & 0.0021 & 0.0234 \\
\hline
\end{tabular}


TABLE XXX

Unlaxial Compression $\eta=1.30:$ SCV displacement field (DX, DY, DZ) in the $z=$ 4.5 plane through the vacancy at site 225 . DY $=0$ for all sites in the $y=4.5$ plane. DX, DY, and DR are expressed in hlc (xy).

\begin{tabular}{|c|c|c|c|c|c|c|c|}
\hline Site & $D X$ & $\mathrm{DZ}$ & $\mathrm{DR}$ & Site & DX & DZ & $\mathrm{DR}$ \\
\hline 471 & 0.0387 & 0.0 & 0.0387 & 221 & 0.0384 & 0.0 & 0.0384 \\
\hline 473 & -0.0306 & 0.0 & 0.0306 & 223 & -0.0265 & 0.0 & 0.0265 \\
\hline 475 & 0.0307 & 0.0 & 0.0307 & 225 & 0.0 & 0.0 & 0.0 \\
\hline 477 & -0.0388 & 0.0 & 0.0388 & 227 & 0.0265 & 0.0 & 0.0265 \\
\hline 479 & 0.0 & 0.0 & 0.0 & 229 & -0.0383 & 0.0 & 0.0383 \\
\hline 421 & 0.0408 & 0.0052 & 0.0411 & 171 & 0.0033 & -0.0658 & 0.0659 \\
\hline 423 & 0.0047 & -0.0427 & 0.0430 & 173 & 0.0719 & 0.1183 & 0.1384 \\
\hline 425 & 0.0 & 0.0480 & 0.0480 & 175 & -0.0720 & 0.1183 & 0.1385 \\
\hline 427 & -0.0046 & -0.0427 & 0.0429 & 177 & -0.0032 & -0.0658 & 0.0659 \\
\hline 429 & -0.0407 & 0.0052 & 0.0411 & 179 & 0.0 & 0.0426 & 0.0426 \\
\hline 371 & 0.0266 & -0.0634 & 0.0688 & 121 & -0.0349 & -0.0195 & 0.0400 \\
\hline 373 & 0.0328 & 0.0051 & $0 . \cup 332$ & 123 & 0.0816 & 0.0427 & 0.0921 \\
\hline 375 & -0.0328 & 0.0051 & 0.0332 & 125 & 0.0 & 0.0284 & 0.0284 \\
\hline 377 & $-0.026 i$ & -0.0634 & 0.0688 & 127 & -0.0816 & 0.0427 & 0.0921 \\
\hline 379 & 0.0 & 0.0700 & 0.0700 & 129 & 0.0348 & -0.0195 & 0.0399 \\
\hline 321 & -0.0349 & 0.0195 & 0.0399 & 71 & 0.0266 & 0.0635 & 0.0689 \\
\hline 323 & 0.0816 & -0.0426 & 0.0921 & 73 & 0.0328 & -0.0051 & 0.0332 \\
\hline 325 & 0.0 & -0.0285 & 0.0285 & 75 & -0.0328 & -0.0051 & 0.0332 \\
\hline 327 & -0.0816 & -0.0426 & 0.0921 & 77 & -0.0267 & 0.0635 & 0.0689 \\
\hline 329 & 0.0348 & 0.0195 & 0.0399 & 79 & 0.0 & -0.0700 & 0.0700 \\
\hline 271 & 0.0033 & 0.0659 & 0.0660 & 21 & 0.0408 & -0.0053 & 0.0411 \\
\hline 273 & 0.0719 & -0.1182 & 0.1384 & 23 & 0.0047 & 0.0428 & 0.0430 \\
\hline 275 & -0.0720 & -0.1182 & 0.1384 & 25 & 0.0 & -0.0479 & 0.0479 \\
\hline 277 & -0.0032 & 0.0659 & 0.0660 & 27 & -0.0046 & 0.0428 & 0.0430 \\
\hline 279 & 0.0 & -0.0427 & 0.0427 & 29 & -0.0407 & -0.0053 & 0.0411 \\
\hline
\end{tabular}


Uniaxial Compression $\eta=1.375$ : SCV displacement field (DX, DY, DZ) in the $y=$ 4.5 plane through the vacancy at site 225 . DY $=0$ for all sites in the $y=4.5$ plane. DX, DY, and DR are expressed in hlc (xy).

\begin{tabular}{|c|c|c|c|c|c|c|c|}
\hline Site & $\mathrm{DX}$ & $\mathrm{DZ}$ & DR & Site & DX & $\mathrm{DZ}$ & $\mathrm{DR}$ \\
\hline 471 & 0.0496 & 0.0 & 0.0496 & 221 & 0.0716 & 0.0 & 0.0716 \\
\hline 473 & -0.0667 & 0.0 & 0.0667 & 223 & -0.0110 & 0.0 & 0.0110 \\
\hline 475 & 0.0668 & 0.0 & 0.0668 & 225 & 0.0 & 0.0 & 0.0 \\
\hline 477 & -0.0496 & 0.0 & 0.0496 & 227 & 0.0109 & 0.0 & 0.0109 \\
\hline 479 & 0.0 & 0.0 & 0.0 & 229 & -0.0716 & 0.0 & 0.0716 \\
\hline $4: 21$ & 0.0445 & 0.0182 & 0.0481 & 171 & 0.0245 & -0.0863 & 0.0897 \\
\hline 423 & -0.0100 & -0.0784 & 0.0790 & 173 & 0.0802 & 0.1022 & 0.1300 \\
\hline 425 & 0.0 & 0.0949 & 0.0949 & 175 & -0.0802 & 0.1022 & 0.1299 \\
\hline 427 & 0.0100 & -0.0784 & 0.0790 & 177 & -0.0245 & -0.0863 & 0.0897 \\
\hline 429 & -0.0444 & 0.0182 & 0.0480 & 179 & 0.0 & 0.0878 & 0.0878 \\
\hline 371 & 0.0036 & -0.0784 & 0.0785 & 121 & -0.0510 & 0.0040 & 0.0511 \\
\hline 373 & 0.0500 & 0.0371 & 0.0622 & 123 & 0.0980 & 0.0115 & 0.0987 \\
\hline 375 & -0.0499 & 0.0371 & 0.0622 & 125 & 0.0 & 0.0101 & 0.0101 \\
\hline 377 & -0.0035 & -0.0784 & 0.0785 & 127 & -0.0981 & 0.0115 & 0.0988 \\
\hline 379 & 0.0 & 0.0858 & 0.0858 & 129 & 0.0510 & 0.0040 & 0.0512 \\
\hline 321 & -0.0510 & -0.0041 & 0.0512 & 71 & 0.0036 & 0.0784 & 0.0784 \\
\hline 323 & 0.0980 & -0.0116 & 0.0987 & 73 & 0.0500 & -0.0372 & 0.0623 \\
\hline 325 & 0.0 & -0.0100 & 0.0100 & 75 & -0.0499 & -0.0372 & 0.0622 \\
\hline 327 & -0.0981 & -0.0116 & 0.0988 & 77 & -0.0035 & 0.0784 & 0.0784 \\
\hline 329 & 0.0510 & -0.0041 & 0.0512 & 70 & 0.0 & -0.0857 & 0.0857 \\
\hline 271 & 0.0245 & 0.0862 & 0.0896 & 21 & 0.0445 & -0.0183 & 0.0481 \\
\hline 273 & 0.0802 & -0.1023 & 0.1300 & 23 & -0.0100 & 0.0784 & 0.0791 \\
\hline 275 & -0.0802 & -0.1023 & 0.1300 & 25 & 0.0 & -0.0949 & 0.0949 \\
\hline 277 & -0.0245 & 0.0862 & 0.0896 & 27 & 0.0100 & 0.0784 & 0.0791 \\
\hline 279 & 0.0 & -0.0879 & 0.0879 & 29 & -0.0444 & -0.0183 & 0.0480 \\
\hline
\end{tabular}


Uniaxial Compression $\eta=1.414:$ SCV displacement field (DX, DY, DZ) in the $y=$ 4.5 plane through the vacancy at site 225 . DY $=0$ for all sites in the $y=4.5$ plane. DX, DY, and DR are expressed in hic (xy).

\begin{tabular}{|c|c|c|c|c|c|c|c|}
\hline Site & $\mathrm{DX}$ & $\mathrm{DZ}$ & DR & Site & DX & $\mathrm{DZ}$ & $\mathrm{DR}$ \\
\hline 471 & -0.0012 & 0.0 & 0.0012 & 221 & 0.0275 & 0.0 & 0.0275 \\
\hline 473 & -0.0052 & 0.0 & 0.0052 & 223 & 0.0288 & 0.0 & 0.0288 \\
\hline 475 & 0.0052 & 0.0 & 0.0052 & 225 & 0.0 & 0.0 & 0.0 \\
\hline 477 & 0.0013 & 0.0 & 0.0013 & 227 & -0.0288 & 0.0 & 0.0288 \\
\hline 479 & 0.0 & 0.0 & 0.0 & 229 & -0.0274 & 0.0 & 0.0274 \\
\hline 421 & 0.0233 & -0.0199 & 0.0306 & 171 & 0.0238 & -0.0246 & 0.0342 \\
\hline 423 & -0.0050 & 0.0061 & 0.0079 & 173 & 0.1022 & 0.0817 & 0.1326 \\
\hline 425 & 0.0 & 0.0156 & 0.0156 & 175 & -0.1044 & 0.0817 & 0.1326 \\
\hline 427 & 0.0051 & 0.0061 & 0.0079 & 177 & 0.0238 & -0.0246 & 0.0342 \\
\hline 429 & -0.0233 & -0.0199 & 0.0307 & 179 & 0.0 & 0.0185 & 0.0185 \\
\hline 371 & 0.0439 & -0.0401 & 0.0658 & 121 & -0.0018 & -0.0074 & 0.0076 \\
\hline 373 & -0.0010 & 0.0250 & 0.0250 & 123 & 0.0814 & 0.0578 & 0.0998 \\
\hline 375 & 0.0010 & 0.0250 & 0.0250 & 125 & 0.0 & -0.0478 & 0.0478 \\
\hline 377 & -0.0439 & -0.0491 & 0.0658 & 127 & -0.0814 & 0.0578 & 0.0999 \\
\hline 379 & 0.0 & 0.0204 & 0.0204 & 129 & 0.0018 & -0.0074 & 0.0076 \\
\hline 321 & -0.0018 & 0.0074 & 0.0076 & 71 & 0.0439 & 0.0491 & 0.0658 \\
\hline 323 & 0.0814 & -0.0578 & 0.0998 & 73 & -0.0010 & -0.0250 & 0.0250 \\
\hline 325 & 0.0 & 0.0478 & 0.0478 & 75 & 0.0010 & -0.0250 & 0.0250 \\
\hline 327 & -0.0814 & -0.0578 & 0.0999 & 77 & -0.0440 & 0.0491 & 0.0659 \\
\hline 329 & 0.0018 & 0.0074 & 0.0076 & 79 & 0.0 & -0.0204 & 0.0204 \\
\hline 271 & 0.0238 & 0.0246 & 0.0342 & 21 & 0.0232 & 0.0199 & 0.0306 \\
\hline 273 & 0.1044 & -0.0817 & 0.1326 & 23 & -0.0050 & -0.0061 & 0.0079 \\
\hline 275 & -0.1044 & -0.0817 & 0.1346 & 25 & 0.0 & -0.0156 & 0.0156 \\
\hline 277 & -0.0238 & 0.0246 & 0.0342 & 27 & 0.0051 & -0.0061 & 0.0079 \\
\hline 279 & 0.0 & -0.0185 & 0.0185 & 29 & -0.0233 & G.0199 & 0.0307 \\
\hline
\end{tabular}


Uniaxial Compression $n=1.50:$ SCV displacement field ( $D X, D Y, D Z$ ) in the $y=$ 4.5 plane through the vacancy at site 225. DY $=0$ for all sites in the $y=4.5$ plane. DX, DY, and DR are expressed in hlc (xy).

\begin{tabular}{|c|c|c|c|c|c|c|c|}
\hline Site & DX & $D Z$ & DR & Site & DX & $\mathrm{DZ}$ & $\mathrm{DR}$ \\
\hline 471 & -0.0219 & 0.0 & 0.0219 & 221 & 0.0204 & 0.0 & 0.0204 \\
\hline 473 & -0.0059 & 0.0 & 0.0059 & 223 & 0.0699 & 0.0 & 0.0699 \\
\hline 475 & 0.0060 & 0.0 & 0.0060 & 225 & 0.0 & 0.0 & 0.0 \\
\hline 477 & 0.0219 & 0.0 & 0.0219 & 227 & -0.0698 & 0.0 & 0.0698 \\
\hline 479 & 0.0 & 0.0 & 0.0 & 229 & $-0.02 C_{4}^{\prime}$ & 0.0 & 0.0204 \\
\hline 421 & 0.0036 & -0.0283 & 0.0285 & 171 & 0.0426 & 0.0118 & 0.0442 \\
\hline 423 & -0.0131 & 0.0179 & 0.0222 & 173 & 0.1144 & 0.0476 & 0.1239 \\
\hline 425 & 0.0 & 0.0176 & 0.0176 & 175 & -0.1144 & 0.0476 & 0.1239 \\
\hline 427 & 0.0130 & 0.0179 & 0.0221 & 177 & -0.0427 & 0.0118 & 0.0443 \\
\hline 429 & -0.0035 & -0.0283 & 0.0285 & 179 & 0.0 & 0.0052 & 0.0052 \\
\hline 371 & 0.0343 & 0.0302 & 0.0457 & 121 & 0.0211 & 0.0162 & 0.0266 \\
\hline 373 & -0.0108 & 0.0339 & 0.0356 & 123 & 0.0675 & 0.0421 & 0.0796 \\
\hline 375 & 0.0109 & 0.0339 & 0.0356 & 125 & 0.0 & -0.0846 & 0.0346 \\
\hline 377 & -0.0344 & -0.0302 & 0.0458 & 127 & -0.0674 & $0.042 I$ & 0.0795 \\
\hline 379 & 0.0 & -0.0279 & 0.0279 & 129 & -0.0212 & 0.0162 & 0.0267 \\
\hline 321 & 0.0211 & -0.0163 & 0.0267 & 71 & 0.0343 & 0.0302 & 0.0457 \\
\hline 323 & 0.0675 & -0.0422 & 0.0796 & 73 & -0.0108 & -0.0338 & 0.0355 \\
\hline 325 & 0.0 & 0.0845 & 0.0845 & 75 & 0.0109 & -0.0338 & 0.0355 \\
\hline 327 & -0.0674 & 0.0422 & 0.0796 & 77 & -0.0344 & 0.0302 & 0.0458 \\
\hline 329 & -0.0212 & -0.0163 & 0.0267 & 79 & 0.0 & 0.0278 & 0.0278 \\
\hline 271 & 0.0436 & -0.0119 & 0.0442 & 21 & 0.0036 & 0.0283 & 0.0285 \\
\hline 273 & 0.1144 & -0.0476 & 0.1239 & 23 & -0.0131 & -0.0178 & 0.0221 \\
\hline 275 & -0.1144 & -0.0476 & 0.1239 & 25 & 0.0 & -0.0177 & 0.0178 \\
\hline 277 & -0.0698 & 0.0 & 0.0698 & 27 & 0.0131 & -0.0178 & 0.0221 \\
\hline 279 & 0.0 & -0.0051 & 0.0051 & 29 & -0.0035 & 0.0283 & 0.0285 \\
\hline
\end{tabular}


Angle $\theta$ between the line from site 173 to site 225 and the displacement vector direction for the atom at site 173 .

\begin{tabular}{|c|c|c|c|c|c|c|}
\hline$n$ & DXX & DY & $\tan \alpha$ & $\alpha$ & $\underline{B}$ & $\theta=\beta-\alpha$ \\
\hline 1.0 & 0.01 & 0.01 & 1.0 & $45^{\circ}$ & $45^{\circ}$ & $0.0^{\circ}$ \\
\hline 1.10 & 0.0188 & 0.0282 & 0.667 & 33.69 & 47.73 & 14.04 \\
\hline 1.20 & 0.0474 & 0.0676 & 0.701 & 35.04 & 50.19 & 15.15 \\
\hline 1.25 & $0.0 \mathrm{il9}$ & 0.1059 & 0.584 & 30.31 & 51.34 & 21.03 \\
\hline 1.30 & 0.7200 & 0.1182 & 0.609 & 31.35 & 52.43 & 21.08 \\
\hline 1.375 & 0.0802 & 0.1023 & 0.784 & 38.10 & 53.97 & 15.87 \\
\hline 1.414 & 0.1044 & 0.0817 & 1.278 & 51.95 & 54.74 & 2.79 \\
\hline 1.50 & 0.1144 & 0.0476 & 2.403 & 67.41 & 56.31 & $-11 \cdot 10$ \\
\hline
\end{tabular}

$\alpha=\tan ^{-1}(\mathrm{DX} / \mathrm{DZ})$

$\beta=\cot ^{-1} n$

C. Split-Vacancy Displacement Field

Figures 44-52 and Tables XXXV-XIIII describe SV displacement fields. Figure 44 provides two examples of the type of displacement field pattern which correlates with the SV being the vacancy migration saddle-point configuration. Figure $44 \mathrm{a}$ is for $\eta=1.0$ isotropic compression and Fig. $44 \mathrm{~b}$ is for $\eta=1.5$ isotropic compression. In both instances, the displacement field pattern is dominated by the displacement vectors of atoms lying on two adjacent [10ī] lines through the two vacant sites. In addition, the displacement vectors for the atoms at firstneighbor sites 173 and 327 are aligned with the [101] 1ine on which these sites are located. As such, the displacement field pattern is a crossed-axis pattern centered on two crossing close-packed line directions. The acute angle between these lines is $90^{\circ}$ and $70.5^{\circ}$, respectively, for an fcc and a bcc crystal. The displacement field of an SV-OP also exhibited a crossed-axis displacement field pattern for $\eta=1.10$ and $\eta=1.20$ uniaxial compression. This is shown in Figs. 45 and 46 . The acute angle between the crossed axes is $84.5^{\circ}$ and $79.6^{\circ}$, respectively, for $\eta=1.10$ and $\eta=1.20$. Close inspection of Figs. 45 and 46 shows that uniaxial compression produced a deviation of the first-neighbor displacement vectors toward the compression axis, but the overall pattern is qualitatively a crossed-axis pattern. In particular, atom displacement vectors in the V-regions 
between the crossed [10̄]] and [101] axes are small and all displacements tend to point toward the vacant sites.

Figure 47 is a map of the SV-OP displacement field for $\eta=1.30$ uniaxial compression. The crossed-axis feature seen in Figs. 44-46 is not present in Fig. 47. Instead, the salient pattern feature of the displacement vectors in Fig. 47 is that they tend to be aligned along either the [101] or the [īo $\overline{1}]$ direction. This particular alignment feature persists in Fig. 48 for $\eta=1.375$ uniaxial compression. This alternating-sign, shear displacement pattern for the SV-OP correlates with it being the stable vacancy configuration.

Figure 49 is an SV-OP displacement field map for $\eta=\sqrt{2}$ uniaxial compression (bcc structure). Because the crystal exhibits cubic symmetry, the SCV is the siable configuration. The crossed-axis displacement pattern clearly exists in this instance and, as stated in Sec. IV, the split-vacancy is the saddle-point configuration for $n=\sqrt{2}$ uniaxial compression.

The $\eta=1.5$ uniaxial compression run either was computationally unstable or reveals yet a third qualitatively distinct displacement field pattern for uniaxial compression. The available evidence appears to support the computational instability interpretation.

Figure 50 is a map of the SV-OP displacement field for $\eta=1.5$ uniaxial compression at the seventh of 20 macro time steps ${ }^{*}$ in the displacement field run for 1.5 compression. As it stands, this displacement field pattern resembles the crossed-axis, bcc crystal structure map of Fig. 49. At the end of the run, however, the displacement field map was that shown in Fig. 51. This "keyhole" pattern is certainly different from the other patterns encountered. Taken together with Fig. 50, it suggests that roughly half of the displacement field follows the crossed-axis model and that the remaining half follows a "circular" pattern, in an $x z-p l a n e$ projection. Note that with only three exceptions, sjtes 227,327 , and 277 , al1 displacement $x$-components are positive. In the planes $y=3.5$ and $y=5.5$ on either side of the $y=4.5$ plane, all displacement $x$-components are negative, and in planes $y=2.5$ and $y=6.5$ all displacement $x$-components are positive. Figure 52 shows the $x y$-plane projection of displacements for each line $z=$ constant in the $y=4.5$ plane. Note that as one moves downward from $z=9.5$

\footnotetext{
* The MOLDY program run on which this section is based ran for 200 time steps. In each instance, a macro time step is equal to 10 time steps. Atom position output was printed at the end of each of 20 macro time steps.
} 
to $z=0.5$, the $y$-components of the first two and the last two displacements in each line are in a direction opposite to that occurring in each adjacent atom line.

A complete set of SV-IP displacement rield tables and maps is not shown in this report because it appears as if the SV-IP does not play a significant role in vacancy migration in a uniaxially compressed crystal. Figures $53-56$ are SV-IP displacement field maps for uniaxial compression $\eta=1.5$.

Figure 53 pertains to the $z=4.5$ plane in which the split-vacancy perturbation center was located. Figures 54 and 55 describe the displacement field in the $z=5.5$ and $z=6.5$ planes. Note the stagnation points at sites 314 and 337 in Figure 55. Atom displacements in the (101) plane, which contains the atom located midway between vacant sites 225 and 226, are plotted in Fig. 56. These four maps are the only ones which were constructed for an in-plane vacancy.

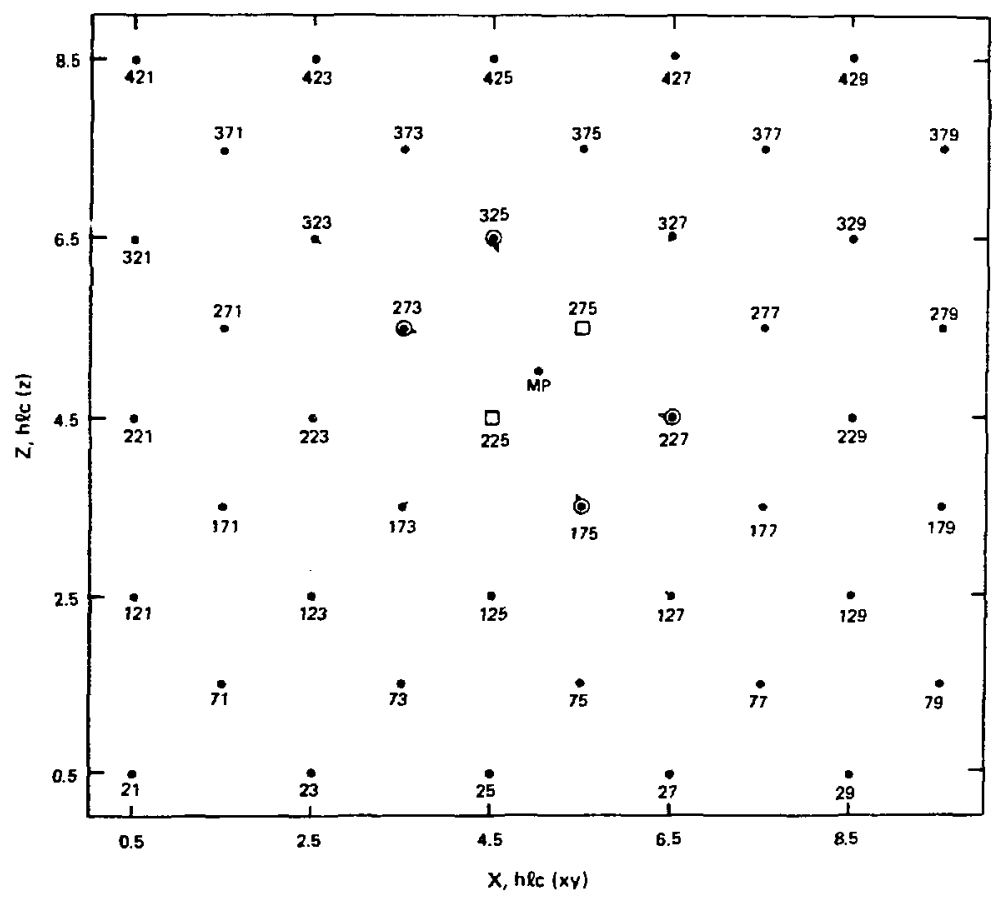

Fig. $44 a$. SV displacement field in the $\mathrm{y}=4.5$ plane. Isotropic compression equal to 1.0 . 

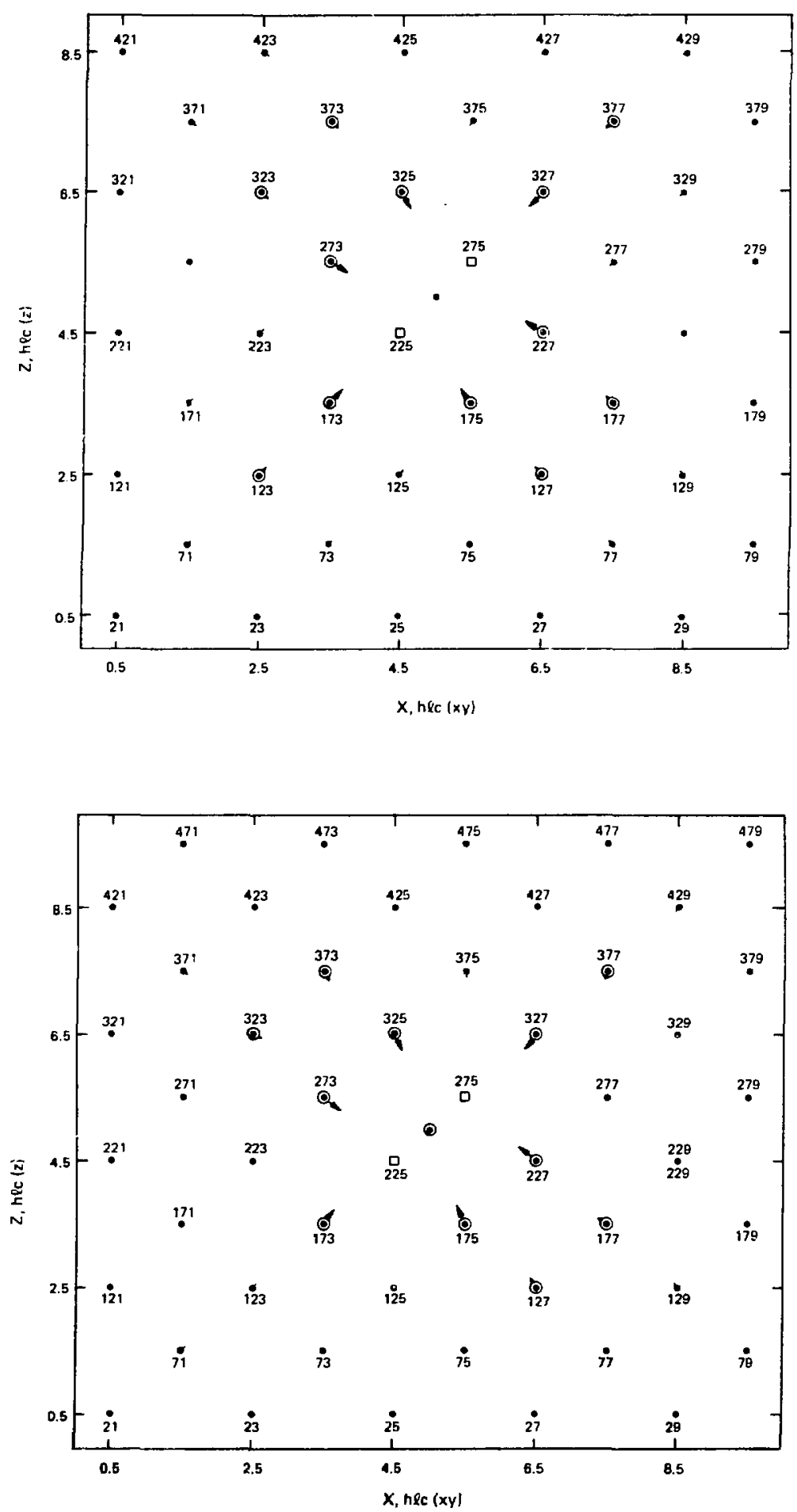

Fig. 45.

SV-OP displacement field in the $y=4.5$ plane. Uniaxial compression equal to 1.10 . 


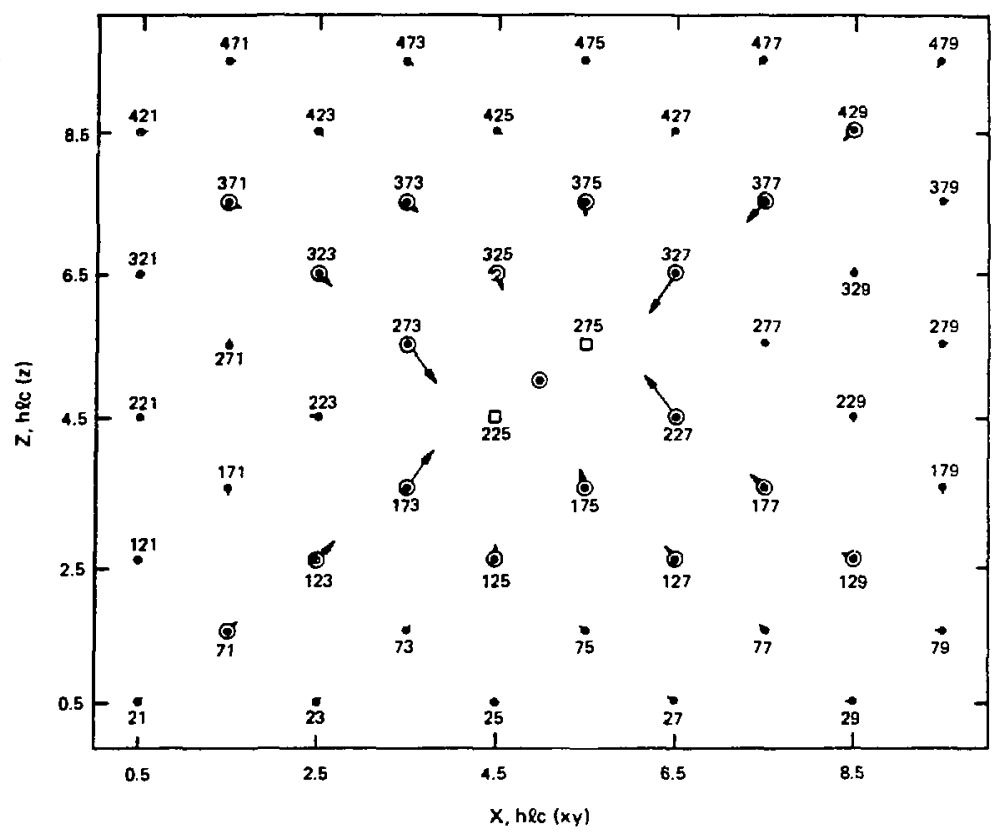

Fig. 46.

SV-OP displacement field in the $y=4.5$ plane. Uniaxial compression equal to 1.20 .

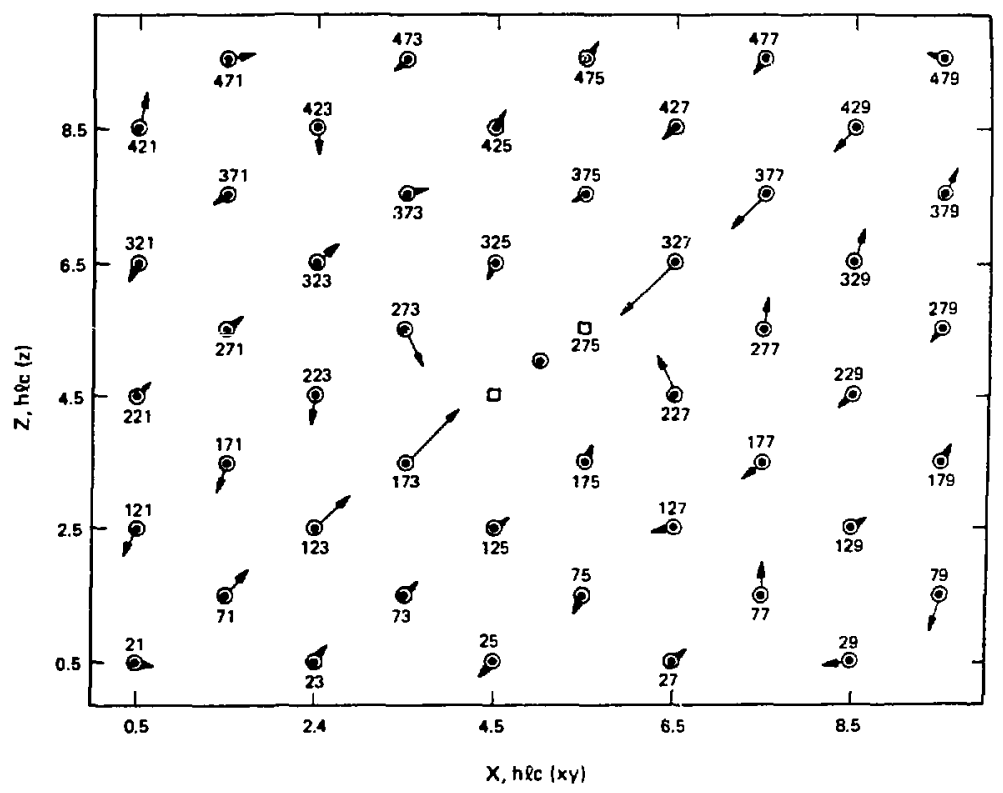

Fig. 47.

SV-OP displacement field in the $y=4.5$ plane. Uniaxial compression equal to 1.30 . 


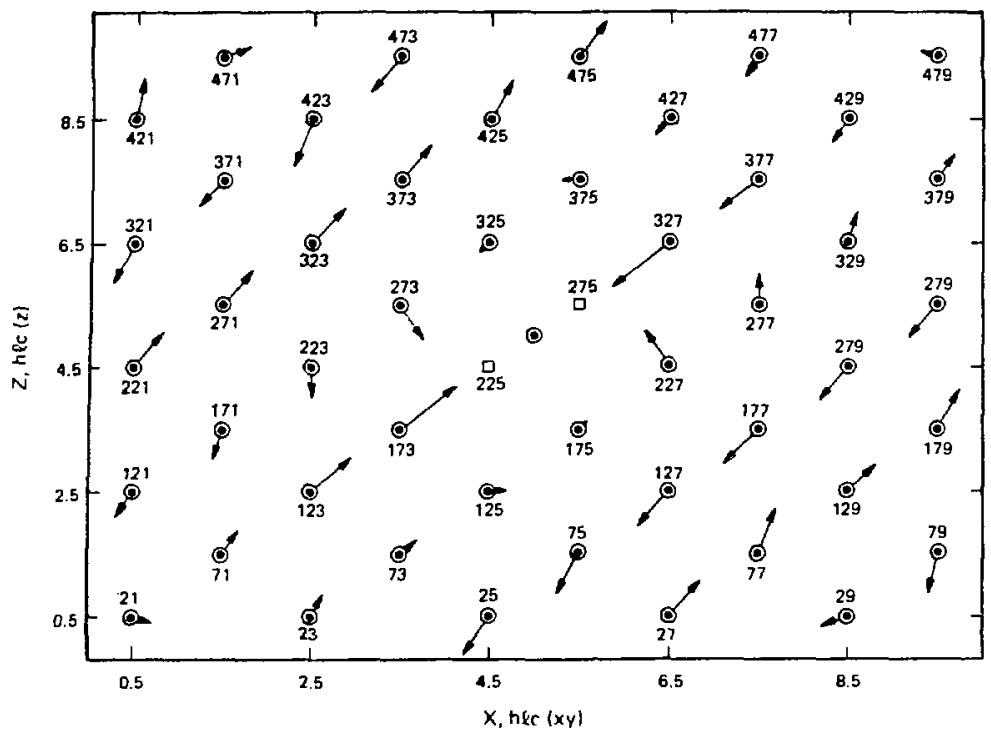

Fig. 48.

SV-OP displacement field in the $\mathrm{y}=4.5$ plane. Uniaxial compression equal to 1.375 .

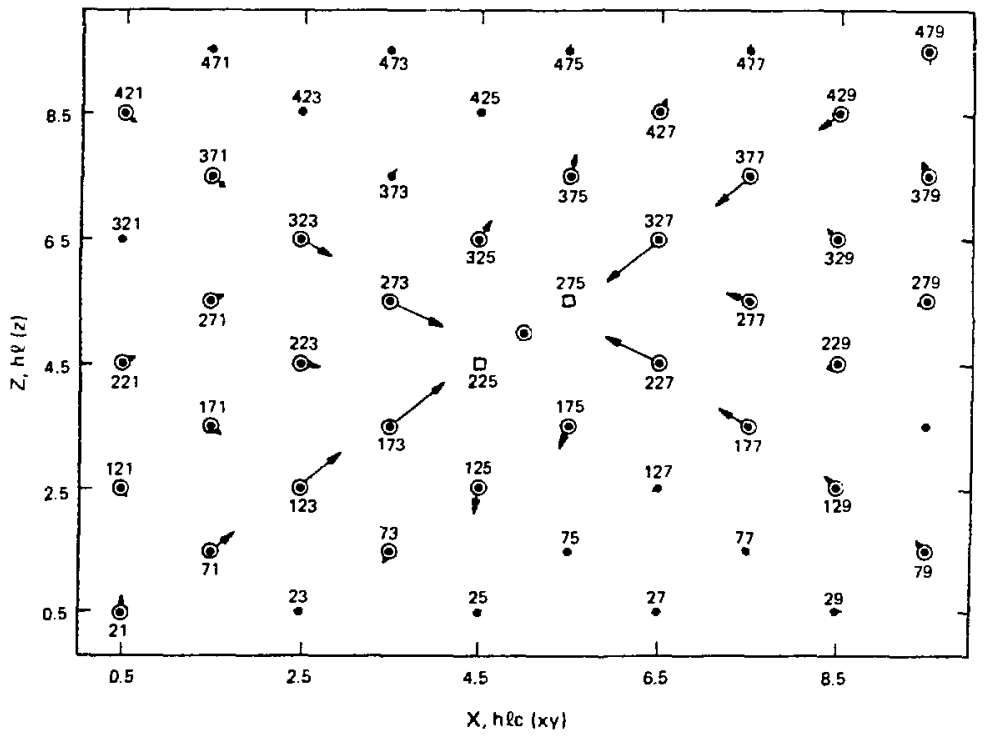

Fig. 49.

SV-OP d-splacement field in the $\mathrm{y}=4.5$ plane. Uniaxial compression equal to 1.414 . 

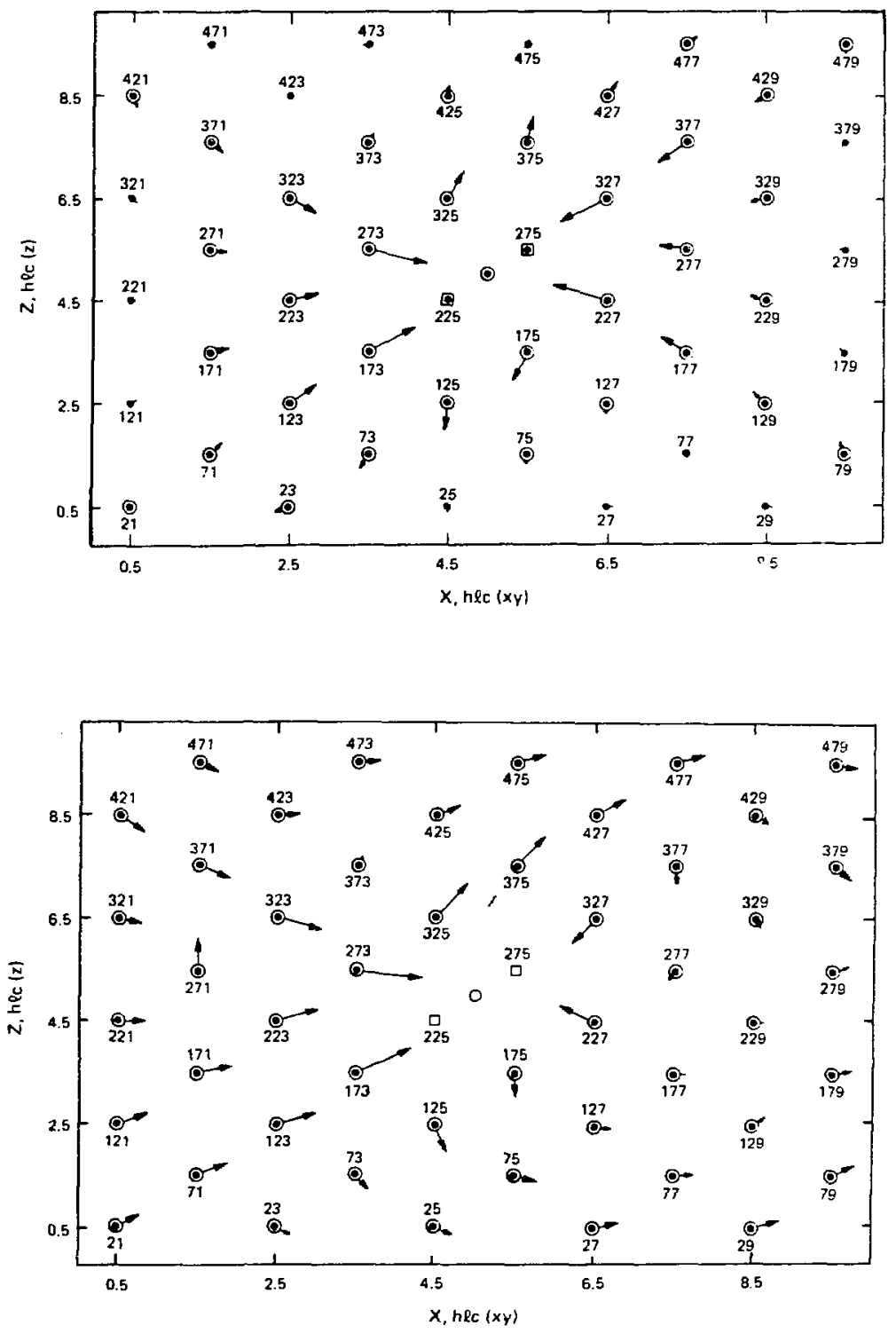

Fig. 50.

SV-OP displacement field in the $y=4.5$ plane. Uniaxial compression equal to 1.50. Seventh iteration, prior to computational instability.
Fig. 51.

SV-OP displacement field in the $y=4.5$ plane. Uniaxial compression equal to 1.50 . Twentieth iteration. 


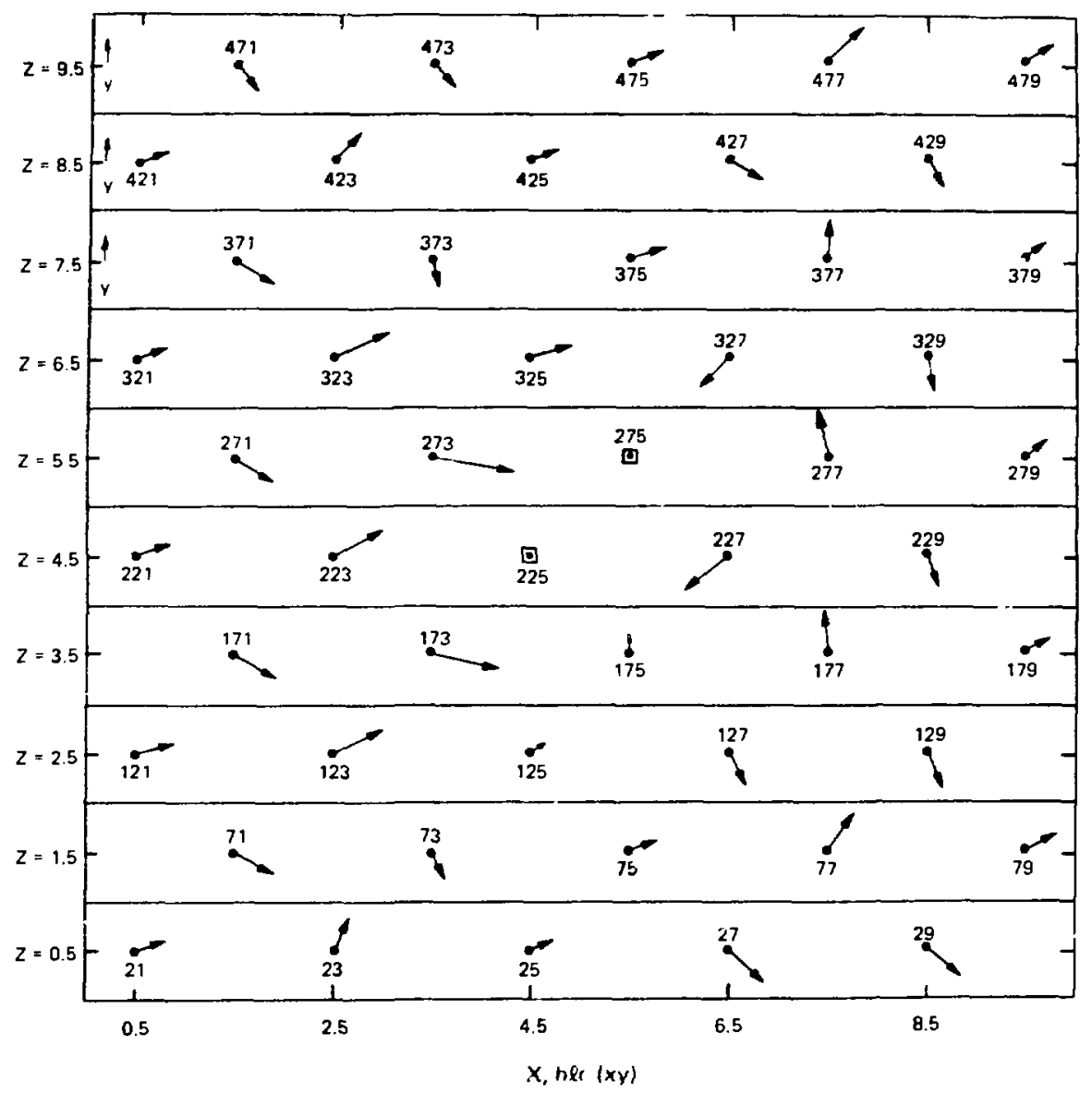

Fig. 52 .

The $x$ - and $y$-displacements for each $z=$ constant line in the $y=$ 4.5 displacement map for uniaxial compression equal to 1.50 . 
Isotropic Compression $\eta=1.0$ : SV displacement field (DX, DY, DZ) in the $y=$ 4.5 plane. Sites 225 and 275 are vacant. The atom initially at site 225 is $10-$ cated at $(5.0,4.5,5.0)$ in hlc units. $D Y=0$ for all sites in the $y=4.5$ plane.

\begin{tabular}{|c|c|c|c|c|c|c|c|}
\hline Site & DX & DZ & DR & Site & $\mathrm{DX}$ & $\mathrm{DZ}$ & $\mathrm{DR}$ \\
\hline 471 & 0.0005 & 0.0001 & 0.0005 & 221 & 0.0005 & 0.0004 & 0.0006 \\
\hline 473 & 0.0005 & -0.0002 & 0.0005 & 223 & 0.0030 & 0.0034 & 0.0045 \\
\hline 475 & -0.0004 & -0.0004 & 0.0006 & 225 & 0.5 & 0.5 & 0.7071 \\
\hline 477 & $-0.000^{n}$ & -0.0006 & 0.0010 & 227 & -0.0294 & 0.0118 & 0.0317 \\
\hline 479 & -0.0003 & -0.0003 & 0.0004 & 229 & -0.0014 & -0.0009 & 0.0014 \\
\hline 421 & -0.0001 & -0.0004 & 0.0004 & 171 & 0.0016 & 0.0016 & 0.0023 \\
\hline 423 & 0.0015 & -0.0010 & 0.0018 & 173 & 0.0095 & 0.0095 & 0.0134 \\
\hline 425 & -0.0003 & -0.0014 & 0.0014 & 175 & -0.0117 & 0.0294 & 0.0316 \\
\hline 427 & -0.0017 & -0.0017 & 0.0024 & 177 & -0.0056 & 0.0048 & 0.0074 \\
\hline 429 & -0.0013 & -0.0013 & 0.0018 & 179 & -0.0002 & 0.0005 & 0.0005 \\
\hline 371 & 0.0010 & -0.0014 & 0.0017 & 121 & 0.0005 & 0.0008 & 0.0009 \\
\hline 373 & 0.0048 & -0.0057 & 0.0074 & 123 & 0.0036 & 0.0036 & 0.0051 \\
\hline 375 & -0.0034 & -0.0030 & 0.0045 & 125 & 0.0034 & 0.0030 & 0.0045 \\
\hline 377 & -0.0035 & -0.0035 & 0.0049 & 127 & -0.0048 & 0.0057 & 0.0074 \\
\hline 379 & --- & -- & --- & 129 & -0.0010 & 0.0015 & 0.0018 \\
\hline 321 & 0.0002 & -0.0006 & 0.0006 & 71 & 0.0012 & $0.00 \geq 2$ & 0.0017 \\
\hline 323 & 0.0057 & -0.0048 & 0.0074 & 73 & 0.0016 & 0.0016 & 0.0023 \\
\hline 325 & 0.0118 & -0.0294 & 0.0317 & 75 & 0.0003 & 0.0015 & 0.0015 \\
\hline 327 & -0.0095 & -0.0095 & 0.0134 & 77 & -0.0014 & 0.0010 & 0.0017 \\
\hline 329 & -0.0017 & -0.0017 & 0.0024 & 79 & 0.0001 & 0.0005 & 0.0005 \\
\hline 271 & 0.0015 & 0.0003 & 0.0015 & 21 & 0.0003 & 0.0003 & 0.0004 \\
\hline 273 & 0.0294 & -0.0117 & 0.0316 & 23 & 0.0008 & 0.0005 & 0.0009 \\
\hline 275 & 0.0 & 0.0 & 0.0 & 25 & 0.0004 & 0.0005 & 0.0606 \\
\hline 277 & -0.0030 & -0.0034 & 0.0045 & 27 & -0.0006 & 0.0002 & 0.0006 \\
\hline 279 & -0.0004 & -0.0004 & 0.0006 & 29 & -0.0004 & -0.0001 & 0.0004 \\
\hline
\end{tabular}


Isotropic Compression $\eta=1.5$ : SV displacement field (DX, DY, DZ) in the $y=4.5$ plane. Sites 225 and 275 are vacant. The atom initially at site 225 is located at $(5.0,4.5,5.0)$ in the hlc units. DY $=0$ for all sites in the $y=4.5$ plane.

\begin{tabular}{|c|c|c|c|c|c|c|c|}
\hline Site & DX & $\mathrm{DZ}$ & $\mathrm{DR}$ & Site & $\mathrm{DX}$ & $\mathrm{DZ}$ & $\mathrm{DR}$ \\
\hline 471 & 0.0039 & -0.0014 & 0.0041 & 221 & 0.0015 & 0.0016 & 0.0022 \\
\hline 473 & 0.0036 & -0.0018 & 0.0040 & 223 & 0.0126 & 0.0063 & 0.0141 \\
\hline 475 & -0.0015 & -0.0016 & 0.0022 & 225 & 0.5 & 0.5 & 0.7071 \\
\hline 477 & -0.0048 & -0.0022 & 0.0053 & 227 & -0.0543 & 0.0309 & 0.0625 \\
\hline 479 & -0.0019 & -0.0019 & 0.0027 & 229 & -0.0059 & 0.0019 & 0.0062 \\
\hline 421 & 0.0015 & -0.0038 & 0.0041 & 171 & 0.0064 & 0.0062 & 0.0089 \\
\hline 423 & 0.0073 & -0.0063 & 0.0096 & 173 & 0.0374 & 0.0374 & 0.0529 \\
\hline 425 & 0.0019 & -0.0058 & 0.0061 & 175 & -0.0308 & 0.0542 & 0.0623 \\
\hline 427 & -0.0062 & -0.0064 & 0.0089 & 177 & -0.0166 & 0.0150 & 0.0224 \\
\hline 429 & -0.0066 & -0.0066 & 0.0093 & 179 & -0.0018 & 0.0036 & 0.0040 \\
\hline 371 & 0.0064 & -0.0074 & 0.0098 & 121 & 0.0022 & 0.0049 & 0.0054 \\
\hline 373 & 0.0150 & -0.0166 & 0.0224 & 123 & 0.0152 & 0.0152 & 0.0215 \\
\hline 375 & -0.0062 & -0.0126 & 0.0140 & 125 & 0.0063 & 0.0126 & 0.0141 \\
\hline 377 & -0.0152 & -0.0152 & 0.0215 & 127 & -0.0151 & 0.0165 & 0.0224 \\
\hline 379 & -0.0022 & -0.0048 & 0.0053 & 129 & -0.0063 & 0.0073 & 0.0096 \\
\hline 321 & 0.0018 & -0.0036 & 0.0040 & 71 & 0.0067 & 0.0067 & 0.0095 \\
\hline 323 & 0.0165 & -0.0151 & 0.0224 & 73 & 0.0062 & 0.0064 & 0.0089 \\
\hline 325 & 0.0309 & -0.0543 & 0.0625 & 75 & -0.0018 & 0.0059 & 0.0062 \\
\hline 327 & -0.0375 & -0.0375 & 0.0530 & 77 & -0.0074 & 0.0064 & 0.0098 \\
\hline 329 & -0.0064 & -0.0062 & 0.0089 & 79 & -0.0014 & 0.0039 & 0.0041 \\
\hline 271 & 0.0059 & -0.0018 & 0.0062 & 21 & 0.0020 & 0.0020 & 0.0028 \\
\hline 273 & 0.0542 & -0.0308 & 0.0623 & 23 & 0.0049 & 0.0022 & 0.0054 \\
\hline 275 & 0.0 & 0.0 & 0.0 & 25 & 0.0016 & 0.0015 & 0.0022 \\
\hline 277 & -0.0126 & -0.0062 & 0.0140 & 27 & -0.0036 & 0.0018 & 0.0040 \\
\hline 279 & -0.0016 & -0.0015 & 0.0022 & 29 & -0.0038 & 0.0015 & 0.0041 \\
\hline
\end{tabular}


Uniaxial Compression $n=1.10:$ SV-OP displacement field (DX, DY, DZ) in the $y=$ 4.5 plane. Sites 225 and $2: 5$ are vacant. The atom initially at site 225 is $10-$ cated at $(5.0,4.5,5.0)$ in hlc units. $D Y=0$ for all sites in the $y=4.5$ plane.

\begin{tabular}{|c|c|c|c|c|c|c|c|}
\hline Site & DX & $\mathrm{DZ}$ & $\mathrm{DR}$ & Site & $\mathrm{DX}$ & DZ & $\mathrm{DR}$ \\
\hline 471 & 0.0044 & -0.0009 & 0.0045 & 221 & -0.0002 & 0.0 & 0.0002 \\
\hline 473 & 0.0034 & -0.0019 & 0.0039 & 223 & -0.0009 & 0.0051 & 0.0052 \\
\hline 475 & -0.0010 & -0.0009 & 0.0013 & 225 & 0.5 & 0.4545 & 0.6757 \\
\hline 477 & -0.0047 & -0.0017 & 0.0050 & 227 & -0.0458 & 0.0417 & 0.0619 \\
\hline 479 & -0.0020 & -0.0014 & 0.0024 & 229 & 0.0013 & -0.0007 & 0.0015 \\
\hline 421 & 0.0026 & -0.0017 & 0.0031 & 171 & 0.0008 & 0.0014 & 0.0016 \\
\hline 423 & 0.0071 & -0.0056 & 0.0090 & 173 & 0.0283 & 0.0423 & 0.0509 \\
\hline 425 & 0.0024 & -0.0053 & 0.0058 & 175 & -0.0240 & 0.0463 & 0.0522 \\
\hline 427 & -0.0048 & 0.0463 & 0.0522 & 177 & -0.0150 & 0.0163 & 0.0222 \\
\hline 429 & -0.0069 & -0.0053 & 0.0087 & 179 & 0.0003 & -0.0004 & 0.0005 \\
\hline 371 & 0.0063 & -0.0059 & 0.0086 & 121 & 0.0006 & 0.0001 & 0.0006 \\
\hline 373 & 0.0142 & -0.0155 & 0.0210 & 123 & 0.0143 & 0.0149 & 0.0206 \\
\hline 375 & -0.0030 & -0.0107 & 0.0111 & 125 & 0.0030 & 0.0106 & 0.0110 \\
\hline 377 & -0.0144 & -0.150 & 0.0208 & 127 & -0.0143 & 0.0156 & 0.0212 \\
\hline 379 & -0.0007 & -0.0001 & 0.0007 & 129 & -0.0062 & 0.0058 & 0.0085 \\
\hline 321 & -0.0002 & 0.0004 & 0.0004 & 71 & 0.0070 & 0.0052 & 0.0087 \\
\hline 323 & 0.0150 & -0.0165 & 0.0223 & 73 & 0.0048 & 0.0054 & 0.0072 \\
\hline 325 & 0.0241 & -0.0464 & 0.0522 & 75 & -0.0024 & 0.0054 & 0.0059 \\
\hline 327 & -0.0283 & 0.0424 & 0.0510 & 77 & -0.0071 & 0.0056 & 0.0090 \\
\hline 329 & -0.0007 & -0.0014 & 0.0016 & 79 & -0.0029 & 0.0016 & 0.0031 \\
\hline 271 & -0.001 .2 & 0.0006 & 0.0013 & 21 & 0.0020 & 0.0015 & 0.0025 \\
\hline 273 & 0.0459 & -0.0417 & 0.0620 & 23 & 0.0047 & 0.0016 & 0.0050 \\
\hline 275 & 0.0 & 0.0 & 0.0 & 25 & 0.0010 & 0.0008 & 0.0013 \\
\hline 277 & 0.0008 & -0.0050 & 0.0051 & 27 & -0.0034 & 0.0018 & 0.0038 \\
\hline 279 & 0.0001 & 0.0 & 0.0001 & 29 & -0.0044 & 0.0008 & 0.0045 \\
\hline
\end{tabular}


Uniaxial Compression $n=1.20$ : SV-OP displacement field (DX, DY, DZ) in the $y=$ 4.5 plane. Sites 225 and 275 are vacant. The atom initially at site 225 is 1ocated at $(5.0,4.5,5.0)$ in hlc units. DY $=0$ for all sites in the $y=4.5$ plane.

\begin{tabular}{|c|c|c|c|c|c|c|c|}
\hline Site & DX & $\mathrm{DZ}$ & $\mathrm{DR}$ & Site & $\mathrm{DX}$ & $\mathrm{DZ}$ & DR \\
\hline 471 & 0.0122 & 0.0023 & 0.0124 & 221 & 0.0008 & -0.0013 & 0.0015 \\
\hline 473 & 0.0054 & -0.0067 & 0.0086 & 223 & -0.0094 & -0.0028 & 0.0098 \\
\hline 475 & 0.0003 & 0.0028 & 0.0028 & 225 & 0.5 & 0.4167 & 0.6509 \\
\hline 477 & -0.0133 & -0.0050 & 0.0142 & 227 & -0.0665 & 0.0875 & 0.1099 \\
\hline 479 & -0.0060 & -0.0056 & 0.0082 & 229 & 0.0322 & -0.0076 & 0.0079 \\
\hline 421 & 0.0122 & 0.0026 & 0.0125 & 171 & -0.0038 & -0.0124 & 0.0130 \\
\hline 423 & 0.0111 & -0.0102 & 0.0151 & 173 & 0.0587 & 0.0894 & 0.1069 \\
\hline 425 & 0.0072 & -0.0062 & 0.0095 & 175 & -0.0089 & 0.0444 & 0.0453 \\
\hline 427 & -0.0114 & -0.0112 & 0.0160 & 177 & -0.0335 & 0.0292 & 0.0444 \\
\hline 429 & -0.0341 & -0.0161 & 0.0282 & 179 & 0.0027 & -0.0071 & 0.0076 \\
\hline 371 & 0.0163 & -0.0126 & 0.0206 & 121 & -0.0023 & -0.0138 & 0.0140 \\
\hline 373 & 0.0180 & -0.0152 & 0.0236 & 123 & 0.0392 & 0.0384 & 0.0549 \\
\hline 375 & -0.0049 & -0.0202 & 0.0208 & 125 & 0.0050 & 0.0203 & 0.0209 \\
\hline 377 & -0.0393 & -0.0383 & 0.0549 & 127 & -0.0180 & 0.0152 & 0.0236 \\
\hline 379 & 0.0022 & 0.0138 & 0.0140 & 129 & -0.0163 & 0.0125 & 0.0205 \\
\hline 321 & -0.0026 & 0.0072 & 0.0076 & 71 & 0.0231 & 0.0162 & 0.0282 \\
\hline 323 & 0.0336 & -0.0292 & 0.0445 & 73 & 0.0115 & 0.0112 & 0.0160 \\
\hline 325 & 0.0089 & -0.0445 & 0.0454 & 75 & -0.0072 & 0.0062 & 0.0095 \\
\hline 327 & -0.0587 & -0.0895 & 0.1070 & 77 & -0.0110 & 0.0102 & 0.0150 \\
\hline 329 & 0.0038 & 0.0125 & 0.0131 & 79 & -0.0122 & -0.0027 & 0.0125 \\
\hline 271 & -0.0022 & 0.0075 & 0.0078 & 21 & 0.0060 & 0.0055 & 0.0081 \\
\hline 273 & 0.0665 & -0.0874 & 0.1098 & 23 & 0.0132 & 0.0051 & 0.0142 \\
\hline 275 & 0.0 & 0.0 & 0.0 & 25 & -0.0002 & -0.0028 & 0.0028 \\
\hline 277 & 0.0095 & 0.0032 & 0.0100 & 27 & -0.0054 & 0.0066 & 0.0085 \\
\hline 279 & -0.0007 & 0.0014 & 0.0016 & 29 & -0.0123 & -0.0024 & 0.0125 \\
\hline
\end{tabular}


TABLE XXXIX

Uniaxial Compression $\eta=1.25$ : SV-OP displacement field (DX, DY, DZ) in the y= 4.5 plane. Sites 225 and 275 are vacant. The atom initially at site 225 is located at $(5.0,4.5,5.0)$ in hlc units. DY $=0$ for all sites in the $y=4.5$

plane.

\begin{tabular}{|c|c|c|c|c|c|c|c|}
\hline Site & $\mathrm{DX}$ & $\mathrm{DZ}$ & $\mathrm{DR}$ & Site & $\mathrm{DX}$ & $\mathrm{DZ}$ & DR \\
\hline 471 & 0.0248 & 0.0083 & 0.0262 & 221 & 0.0064 & 0.0027 & 0.0069 \\
\hline 473 & 0.0014 & -0.0108 & 0.0109 & 223 & -0.0127 & -0.0260 & 0.0289 \\
\hline 475 & 0.0036 & 0.0083 & 0.0090 & 225 & 0.5 & 0.4 & 0.6403 \\
\hline 477 & -0.0212 & -0.0157 & 0.0264 & 227 & -0.0642 & 0.1016 & 0.1202 \\
\hline 479 & -0.0130 & -0.0050 & 0.0139 & 229 & -0.0061 & -0.0129 & 0.0143 \\
\hline 421 & 0.0201 & 0.0218 & 0.0296 & 171 & -0.0114 & -0.0352 & 0.0370 \\
\hline 423 & 0.0106 & -0.0198 & 0.0225 & 173 & 0.0830 & 0.1075 & 0.1358 \\
\hline 425 & 0.0095 & -0.0063 & 0.0095 & 175 & 0.0043 & 0.0430 & 0.0432 \\
\hline 427 & -0.0192 & -0.0194 & 0.0273 & 177 & -0.0413 & 0.0216 & 0.0466 \\
\hline 429 & -0.0356 & -0.0310 & 0.0472 & 179 & 0.0056 & -0.0034 & 0.0066 \\
\hline 371 & 0.0131 & -0.0138 & 0.0190 & 121 & -0.0125 & -0.0327 & 0.0350 \\
\hline 373 & 0.0217 & $-0.0106 ́$ & 0.0242 & 123 & 0.0600 & 0.0590 & 0.0841 \\
\hline 375 & -0.0130 & -0.0240 & 0.0273 & 125 & 0.0130 & 0.0238 & 0.0271 \\
\hline 377 & -0.0600 & -0.0589 & 0.0841 & 127 & -0.0216 & 0.0106 & 0.0241 \\
\hline 379 & 0.0124 & 0.0327 & 0.0350 & 129 & -0.0130 & 0.0137 & 0.0189 \\
\hline 321 & -0.0056 & 0.0034 & 0.0066 & 71 & 0.0356 & 0.0310 & 0.0472 \\
\hline 323 & 0.0412 & -0.0216 & 0.0465 & 73 & 0.0191 & 0.0194 & 0.0272 \\
\hline 325 & -0.0042 & -0.0430 & 0.0432 & 75 & -0.0095 & 0.0002 & 0.0095 \\
\hline 327 & -0.0831 & -0.1074 & 0.1358 & 77 & -0.0106 & 0.0198 & 0.0224 \\
\hline 329 & 0.0113 & 0.0351 & 0.0369 & 79 & -0.0201 & -0.0218 & 0.0296 \\
\hline 271 & 0.0061 & 0.0128 & 0.0142 & 21 & 0.0131 & 0.0049 & 0.0140 \\
\hline 273 & 0.0642 & -0.1016 & 0.1202 & 23 & 0.0211 & 0.0158 & 0.0264 \\
\hline 275 & 0.0 & 0.0 & 0.0 & 25 & -0.0036 & -0.0084 & 0.0091 \\
\hline 277 & 0.0126 & 0.0261 & 0.0290 & 27 & -0.0014 & 0.0108 & 0.0109 \\
\hline 279 & -0.0065 & -0.0026 & 0.0070 & 29 & -0.0248 & -0.0084 & 0.0262 \\
\hline
\end{tabular}


TABLE XL

Uniaxial Compression $\eta=1.375$ : SV-OP displacement field (DX, DY, DZ) in the $y=$ 4.5 plane. Sites 225 and 275 are vacant. The atom initially at site 225 is located at $(5.0,4.5,5.0)$ in hlc units. DY $=0$ for all sites in the $y=4.5$ plane.

\begin{tabular}{|c|c|c|c|c|c|c|c|}
\hline Site & $\mathrm{DX}$ & $\mathrm{DZ}$ & DR & Site & $\mathrm{DX}$ & $\mathrm{Dz}$ & $\mathrm{DR}$ \\
\hline 471 & 0.0578 & 0.0220 & 0.0618 & 221 & 0.0658 & -0.0768 & 0.1011 \\
\hline 473 & -0.0661 & -0.0767 & 0.1012 & 223 & 0.0012 & 0.0688 & 0.0688 \\
\hline 475 & 0.0569 & 0.0853 & 0.1025 & 225 & 0.5 & 0.3636 & 0.6182 \\
\hline 477 & -0.0296 & -0.0533 & 0.0610 & 227 & -0.0404 & 0.0751 & 0.0852 \\
\hline 479 & -0.0365 & 0.0092 & 0.0376 & 229 & -0.0748 & -0.0766 & 0.1070 \\
\hline 421 & 0.0238 & 0.0882 & 0.0913 & 171 & -0.0195 & -0.0711 & 0.0737 \\
\hline 423 & $-0.064 !$ & -0.1059 & 0.1147 & 173 & 0.1321 & 0.1029 & 0.1674 \\
\hline 425 & 0.0463 & 0.0945 & 0.1052 & 175 & 0.0226 & 0.0151 & 0.0272 \\
\hline 427 & -0.0349 & -0.0279 & 0.0447 & 177 & -0.0783 & -0.0787 & 0.1109 \\
\hline 429 & -0.0423 & -0.0594 & 0.0729 & 179 & 0.0510 & 0.0913 & $0.1 \cap 46$ \\
\hline 371 & -0.0633 & -0.0646 & 0.0904 & 121 & -0.0394 & -0.0583 & 0.0704 \\
\hline 373 & 0.0686 & 0.0767 & 0.1029 & 123 & 0.0883 & -0.0747 & 0.1156 \\
\hline 375 & -0.0372 & 0.0028 & 0.0373 & 125 & 0.0372 & -0.0028 & 0.0373 \\
\hline 377 & -0.0884 & -0.0747 & 0.1157 & 127 & -0.0687 & -0.0767 & 0.1030 \\
\hline 379 & 0.0393 & 0.0583 & 0.0703 & 129 & 0.0633 & 0.0646 & 0.0904 \\
\hline 321 & -0.0510 & -0.0913 & 0.1046 & 71 & 0.0422 & $0 . C 594$ & 0.0729 \\
\hline 323 & 0.0784 & 0.0786 & 0.1110 & 73 & 0.0350 & 0.0279 & 0.0448 \\
\hline 325 & -0.0226 & -0.0151 & 0.0272 & 75 & -0.0464 & -0.0945 & 0.1053 \\
\hline 327 & -0.1322 & -0.1031 & 0.1676 & 77 & 0.0441 & 0.1059 & 0.1147 \\
\hline 329 & 0.0195 & 0.0711 & 0.0737 & 79 & -0.0238 & -0.0881 & 0.0913 \\
\hline 271 & 0.0749 & 0.0767 & 0.1072 & 21 & 0.0364 & -0.0094 & 0.0376 \\
\hline 273 & 0.0403 & -0.0751 & 0.0852 & 23 & 0.0296 & 0.0533 & 0.0610 \\
\hline 275 & 0.0 & 0.0 & 0.0 & 25 & -0.0568 & -0.0855 & 0.1026 \\
\hline 277 & -0.0011 & 0.0688 & 0.0688 & 27 & 0.0662 & 0.0767 & 0.1013 \\
\hline 279 & -0.0658 & -0.0768 & 0.1011 & 29 & -0.0577 & -0.0220 & 0.0618 \\
\hline
\end{tabular}


Uniaxial Compression $n=1.414:$ SV-OP displacement field (DX, DY, DZ) in the $y=4.5$ plane. Sites 225 and 275 are vacant. The atom initially at site 225 is located at $(5.0,4.5,5.0)$ in the hlc units. $D Y=0$ for all sites in the $y=4.5$ plane.

\begin{tabular}{|c|c|c|c|c|c|c|c|}
\hline Site & DX & $\mathrm{D} 2$ & $\mathrm{DR}$ & Site & $\mathrm{DX}$ & $\mathrm{DZ}$ & \\
\hline 471 & -0.0100 & 0.0033 & 0.0105 & 221 & 0.0152 & 0.0066 & 0.0166 \\
\hline 473 & 0.0002 & -0.0032 & 0.0032 & 223 & 0.0423 & -0.0072 & 0.0429 \\
\hline 475 & 0.0 & 0.0081 & 0.0081 & 225 & 0.5 & 0.3536 & 0.6124 \\
\hline 477 & 0.0048 & 0.0188 & 0.0194 & 227 & -0.1161 & 0.0584 & 0.1300 \\
\hline 479 & -0.0040 & -0.0284 & 0.0286 & 229 & -0.0273 & -0.0095 & 0.0289 \\
\hline 421 & 0.0160 & -0.0165 & 0.0230 & 171 & 0.0221 & -0.0160 & 0.0273 \\
\hline 423 & -0.0016 & 0.0037 & 0.0040 & 173 & 0.1238 & 0.0969 & 0.1572 \\
\hline 425 & -0.0007 & 0.0068 & 0.0068 & 175 & -0.0219 & -0.0399 & 0.0455 \\
\hline 427 & 0.0095 & 0.0269 & 0.0285 & 177 & -0.0660 & 0.0363 & 0.0753 \\
\hline 429 & -0.0526 & -0.0443 & 0.0688 & 179 & 0.0020 & 0.0017 & 0.0026 \\
\hline 371 & 0.0313 & -0.0318 & 0.0446 & 121 & 0.0083 & -0.0157 & 0.0178 \\
\hline 373 & 0.0078 & 0.0123 & 0.0146 & 123 & 0.0889 & 0.0759 & 0.1169 \\
\hline 375 & 0.0108 & 0.0492 & 0.0504 & 125 & -0.0107 & -0.0492 & 0.0504 \\
\hline 377 & -0.0888 & -0.0762 & 0.1170 & 127 & -0.0079 & -0.0124 & 0.0147 \\
\hline 379 & -0.0084 & 0.0158 & 0.0179 & 129 & -0.0313 & 0.0318 & 0.0446 \\
\hline 321 & -0.0020 & -0.0017 & 0.0026 & 71 & 0.0524 & 0.0443 & 0.0686 \\
\hline 323 & 0.0659 & -0.0365 & 0.0753 & 73 & -0.0094 & -0.0269 & 0.0285 \\
\hline 325 & 0.0219 & 0.0399 & 0.0455 & 75 & 0.0006 & -0.0068 & 0.0068 \\
\hline 327 & -0.1239 & -0.0969 & 0.1573 & 77 & 0.0017 & -0.0038 & 0.0042 \\
\hline 329 & -0.0221 & 0.0160 & 0.0273 & 79 & -0.0160 & 0.0167 & 0.0231 \\
\hline 271 & 0.0272 & 0.0096 & 0.0288 & 21 & 0.0039 & 0.0283 & 0.0286 \\
\hline 273 & 0.1161 & -0.0584 & 0.1300 & 23 & -0.0048 & -0.0187 & 0.0193 \\
\hline 275 & 0.0 & 0.0 & 0.0 & 25 & -0.0001 & -0.0082 & 0.0082 \\
\hline 277 & -0.0424 & 0.0074 & 0.0430 & 27 & -0.0001 & 0.0034 & 0.0034 \\
\hline 279 & -0.0152 & -0.0066 & 0.0166 & 29 & 0.0100 & -0.0032 & 0.0105 \\
\hline
\end{tabular}


Uniaxia1 Compression $\eta=1.50$ : SV-OP displacement field (DX, DY, DZ) in the y= 4.5 plane at the seventh macro time step. Sites 225 and 275 are vacant. The atom initially at site 225 is located at $(5.0,4.5,5.0)$ in hlc units. DY $=0$ for all sites in the $y=4.5$ plane.

\begin{tabular}{|c|c|c|c|c|c|c|c|}
\hline Site & DX & D2 & DR & Site & $\mathrm{DX}$ & $\mathrm{DZ}$ & $\mathrm{DR}$ \\
\hline 471. & -0.0149 & -0.0023 & 0.0151 & 221 & 0.0131 & 0.0012 & 0.0132 \\
\hline 473 & -0.0080 & 0.0011 & 0.0081 & 223 & 0.0737 & 0.0077 & 0.0741 \\
\hline 475 & 0.0037 & 0.0146 & 0.0151 & 225 & 0.5 & 0.3333 & 0.6009 \\
\hline 477 & 0.0153 & 0.0135 & 0.0204 & 227 & -0.1449 & 0.0400 & 0.1503 \\
\hline 479 & 0.0011 & -0.0204 & 0.0204 & 229 & -0.0380 & 0.0070 & 0.0386 \\
\hline 421 & 0.0076 & -0.0261 & 0.0272 & 171 & 0.0387 & 0.0103 & 0.0400 \\
\hline 423 & -0.0015 & 0.0050 & 0.0052 & 173 & 0.1156 & 0.0614 & 0.1309 \\
\hline 425 & -0.0036 & 0.0170 & 0.0174 & 175 & -0.0373 & -0.0734 & 0.0823 \\
\hline 427 & 0.0157 & 0.0318 & 0.0355 & 177 & -0.0708 & 0.0394 & 0.0810 \\
\hline 429 & -0.0287 & -0.0312 & 0.0424 & 179 & -0.0105 & 0.0083 & 0.0134 \\
\hline 371 & 0.0339 & -0.0283 & 0.0422 & 121 & 0.0125 & 0.0131 & 0.0181 \\
\hline 373 & 0.0092 & 0.0154 & 0.0179 & 123 & 0.0664 & 0.0478 & 0.0818 \\
\hline 375 & 0.0149 & 0.0731 & 0.0746 & 125 & -0.0148 & -0.0731 & 0.0746 \\
\hline 377 & -0.0663 & -0.0478 & 0.0817 & 127 & -0.0090 & -0.0154 & 0.0178 \\
\hline 379 & -0.0125 & -0.0132 & 0.0182 & 129 & -0.0337 & 0.0284 & 0.0441 \\
\hline 321 & 0.0107 & -0.0082 & 0.0135 & 71 & 0.0288 & 0.0311 & 0.0424 \\
\hline 323 & 0.0709 & -0.0395 & 0.0812 & 73 & -0.0157 & -0.0318 & 0.0355 \\
\hline 325 & 0.0374 & 0.0734 & 0.0824 & 75 & 0.0037 & -0.0170 & 0.0174 \\
\hline 327 & -0.1155 & -0.0613 & 0.1308 & 77 & 0.0017 & -0.0051 & 0.0054 \\
\hline 329 & -0.0386 & -0.0104 & 0.0400 & 79 & -0.0075 & 0.0262 & 0.0272 \\
\hline 271 & 0.0382 & -0.0071 & 0.0388 & 21 & -0.0009 & 0.0205 & 0.0205 \\
\hline 273 & 0.1450 & -0.0400 & 0.1504 & 23 & -0.0152 & -0.0136 & 0.0204 \\
\hline 275 & 0.0 & 0.0 & 0.0 & 25 & -0.0036 & -0.0146 & 0.0150 \\
\hline 277 & -0.0735 & -0.0078 & 0.0739 & 27 & 0.0081 & -0.0010 & 0.0082 \\
\hline 279 & -0.0129 & -0.0012 & 0.0130 & 29 & 0.0150 & 0.0022 & 0.0152 \\
\hline
\end{tabular}


Uniaxial Compression $\eta=1.50$ : SV-OP displacement field (DX, DY, DZ) in the $y=$ 4.5 plane at the 20 th macro time step. Sites 225 and 275 are vacant. The atom initially at site 225 is located at $(5.0,4.5,5.0)$ in hlc units. $D Y=0$ for all sites in the $\mathrm{y}=4.5$ plane.

\begin{tabular}{|c|c|c|c|c|c|c|c|}
\hline Site & $\mathrm{DX}$ & $\mathrm{DZ}$ & $\mathrm{DR}$ & Site & DX & $\mathrm{DZ}$ & $\mathrm{DR}$ \\
\hline $47 . L$ & 0.0370 & -0.0207 & 0.0424 & 221 & 0.0709 & 0.0306 & 0.0772 \\
\hline 473 & 0.0432 & -0.0025 & 0.0433 & 223 & 0.1075 & 0.0202 & 0.1094 \\
\hline 475 & 0.0676 & 0.0194 & 0.0703 & 225 & 0.5 & 0.3333 & 0.6009 \\
\hline 477 & 0.0706 & 0.0226 & 0.0741 & 227 & -0.0858 & 0.0273 & 0.0900 \\
\hline 479 & 0.0619 & -0.0122 & 0.0631 & 229 & 0.0184 & 0.0035 & 0.0187 \\
\hline 421 & 0.0554 & -0.0368 & 0.0665 & 171 & 0.0897 & 0.0220 & 0.0924 \\
\hline 423 & 0.0507 & -0.0050 & 0.0509 & 173 & 0.1375 & 0.0604 & 0.1502 \\
\hline 425 & 0.0546 & 0.0223 & 0.0590 & 175 & 0.0031 & -0.0645 & 0.0646 \\
\hline 427 & 0.0745 & 0.0388 & 0.0840 & 177 & -0.0065 & 0.0219 & 0.0228 \\
\hline 429 & 0.0339 & -0.0218 & 0.0403 & 179 & 0.0513 & 0.0138 & 0.0531 \\
\hline 371 & 0.0783 & -0.0283 & 0.0832 & 121 & 0.0754 & 0.0286 & 0.0806 \\
\hline 373 & 0.0610 & 0.0155 & 0.0629 & 123 & 0.1050 & 0.0441 & 0.1139 \\
\hline 375 & 0.0738 & 0.0797 & 0.1086 & 125 & 0.0289 & -0.0709 & 0.0766 \\
\hline 377 & -0.0024 & -0.0361 & 0.0362 & 127 & 0.0392 & -0.0120 & 0.0410 \\
\hline 379 & 0.0399 & -0.0270 & 0.0482 & 129 & 0.0337 & 0.0209 & 0.0398 \\
\hline 321 & 0.0641 & -0.0138 & 0.0656 & 71 & 0.0797 & 0.0326 & 0.0861 \\
\hline 323 & 0.1088 & -0.0290 & 0.1126 & 73 & 0.0286 & -0.0375 & 0.0472 \\
\hline 325 & 0.0820 & 0.0868 & 0.1194 & 75 & 0.0562 & -0.0178 & 0.0590 \\
\hline 327 & -0.0587 & -0.0590 & 0.0832 & 77 & 0.0537 & 0.0042 & 0.0539 \\
\hline 329 & 0.0137 & -0.0205 & 0.0247 & 79 & 0.0646 & 0.0312 & 0.0717 \\
\hline 271 & 0.0839 & -0.0019 & 0.0839 & 21 & 0.0653 & 0.0174 & 0.0676 \\
\hline 273 & 0.1737 & -0.0253 & 0.1755 & 23 & 0.0289 & -0.0222 & 0.0364 \\
\hline 275 & 0.0 & 0.0 & 0.0 & 25 & 0.0524 & -0.0167 & 0.0550 \\
\hline 277 & -0.0206 & -0.0154 & 0.0257 & 27 & 0.0659 & 0.0065 & 0.0662 \\
\hline 279 & 0.0450 & -0.0058 & 0.0454 & 29 & 0.0718 & 0.0163 & 0.0736 \\
\hline
\end{tabular}



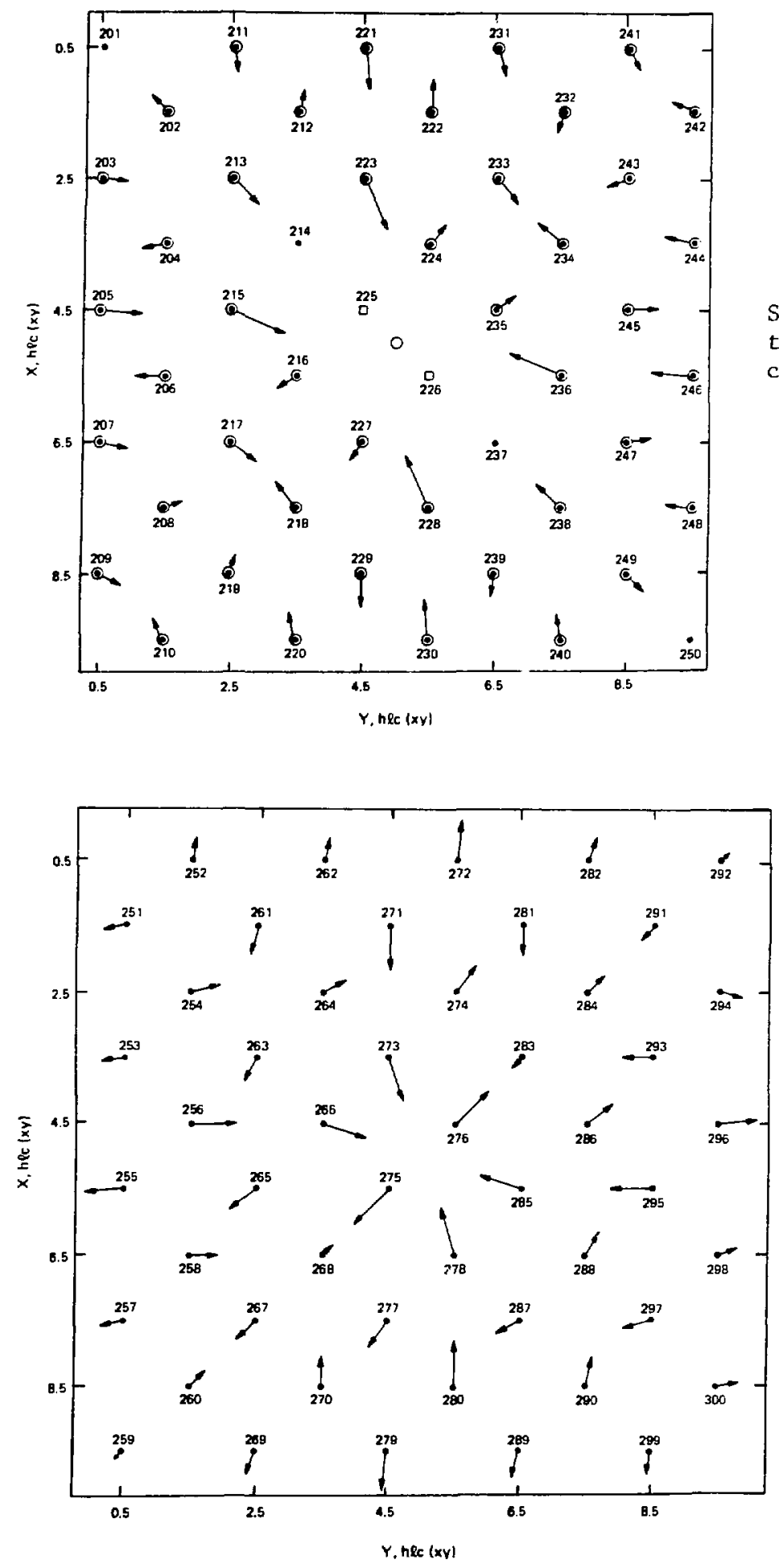

Fig. 53.

SV-IP displacement field in the $\mathrm{z}=4.5$ plane. Uniaxial compression equal to 1.50 .
Fig. 54 .

SV-IP displacement field in the $z=5.5$ plane. Uniaxial compression equal to 1.50 . 


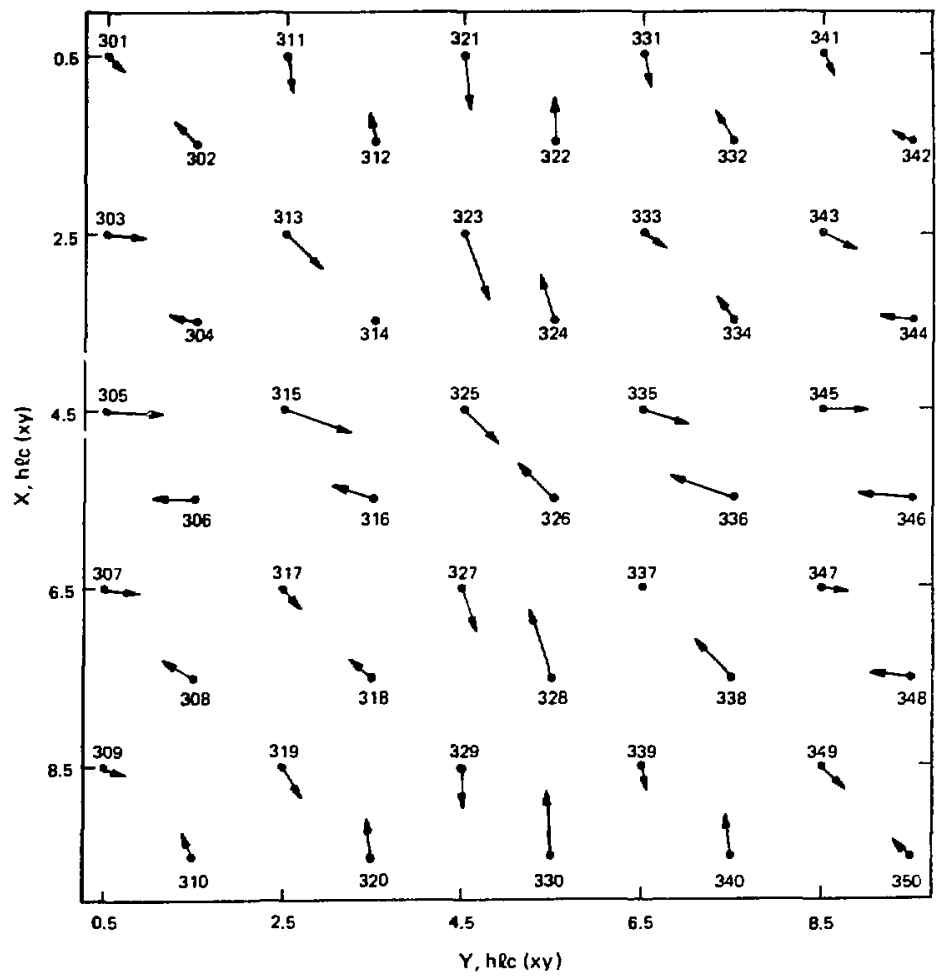

Fig. 55 .

SV-IP displacement field in the $z=6.5$ plane. Uniaxial compression equal to 1.50 .

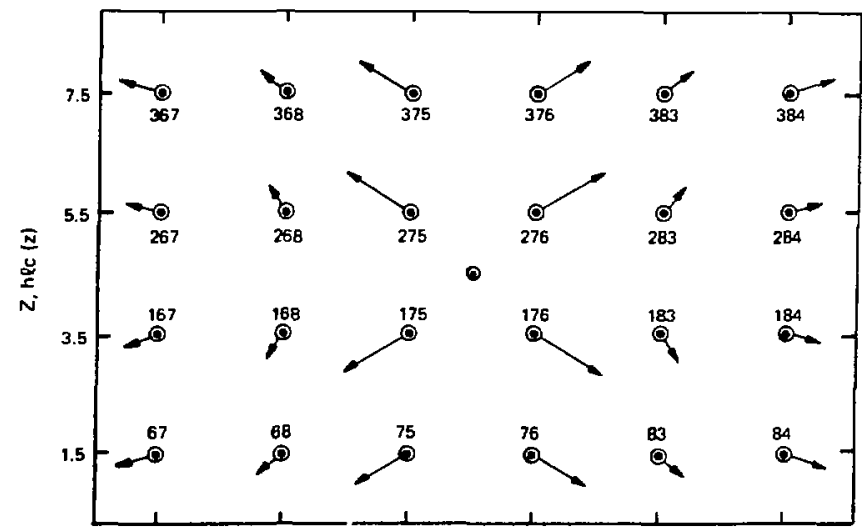

Fig. 56.

SV-IP displacement field in the (110) plane through the midpoint atom. Uniaxial compression equal to 1.50 . 
VI. SUMMARY AND CONCLUSIONS

This study consisted of running static equilibrium calculations for a vacancy in an fcc Lennard-Jones crystal subjected to (1) isotropic compression in one instance, and (2) uniaxial compression along the [001] axis in the second instance The SCV and SV configurations were considered in the case of isotropic compression. Three vacancy configurations were studied in the case of uniaxial compression; these were the SCV, SV-IP, and SV-OP. The compression ranges considered for isotropic compression and uniaxial compression were $0.94 \leqslant \eta \leqslant 1.50$ and $1.0 \leqslant$ $n \leqslant 1.5$, respectively.

The purposes of the study were (1) to characterize vacancy migration in a un axially compressed crystal for use in interpreting shock process simulation results for a crystal containing vacancy defects, and (2) to identify seeming defect displacement field "properties" that might be artifacts of imposing periodic boundary conditions in the computer experiments. In general, a eomputer experiment study of defect migration involves (I) discovering what is the stable defect configuration, and (2) discovering what is the saddle-point configuration for the migration of the defect.

If the involved defect is a point defect, one usually can guess what is the stable defect configuration and can reduce the number of saddle-point possibilities to two or three on the basis of crystal symmetry. If, however, the defect is not a point defect, there is currently no way to determine uniquely either the stable defect configuration or the saddle-point configuration. Because of these circumstances, the initial exploration of either a vacancy or an interstitial defect proceeds on the assumption that the defect will, in fact, behave like a point defect. This tactic normally is profitable in the sense that it usually gives a qualitatively correct picture of the "actual" migration process, even though the defect concerned is not properly a point defect.

A light-hearted definition of a point defect is: A point defect is a defect that is easy to work with in a computer experiment. In many ways this statement is close to the truth. Let us call the part of a defect displacement field in which the atom displacenents are greater than or equal to $1 \%$ of the interatomic distance the GE1\% displacement field. From experience we know that defect migration calculations normally are difficult when the defect's GE1\% displacement field exceeds $100 \mathrm{av}$, where av stands for atomic volume. This is true because a migration calculation ultimately reduces to potential energy surface mapping and when the defect displacement field is extensive, each of a myriad atom-position 
arrangements will correspond to a given crystal energy stipulation. In effect, a defect is a point defect if one can characterize its migration process by characterizing the movement of a single atom in the defect core. When the movement of two or more atoms in the defect core needs to be characterized in a migration calculation, the defect is, in effect, an extended defect and is intractable.

In the case of the fcc Lennard-Jones crystal concerned, it was found that the stable vacancy configuration is a function of the uniaxial compression magnitude. For $1.0 \leqslant n \leqslant 1.29$, the $\mathrm{SCV}$ is the stable configuration, and for $1.29 \leqslant n$ $\leqslant 1.5$, the SV-OP is the stable configuration. The SV-IP was not found to be the stable vacancy configuration in any of the uniaxial compression runs. In the case of isotropic compression, the SCV was the stable vacancy configuration for $0.94 \leqslant n \leqslant 1.5$.

Purely repulsive atom pair-potential energy functions were used in many of the early computer experiments on defect properties. In particular, Vineyard (1961) shows picti es of the vacancy cluster configurations that are predicted when a purely repulsive atom pair interaction is assumed. In each of these examples, the vacancy cluster configuration energy is lowered by the intrusion of atoms, initially at the cluster surface, into the central portion of an initially empty, compact void. One example is a divacancy configuration in an fcc crystal that consists of four vacant atom sites at the corners of a tetrahedron, inside of which are located two intruded atoms. A similar effect occurs in the case of the SV-OP. In this instance, we have two vacant sites with an atom between them. The intruded atom feature of vacancy defects when the atom interaction is repulsive is part, but not all, of the reason for the SV-OP becoming the stable vacancy configuration. It cannot be the only reason because the SCV still is the stable vacancy form in isotropic compression at $\eta=1.5$. The repulsive interaction, intruded atom effect is assisted by the asymmetry provided by uniaxial compression. The resolved stress along shear displacements favorable to SV-OP formation.

As shown in Fig. 43, the angle $\theta$ between the displacement vector for an atom at a first-neighbor site $\mathrm{cf}$ an SCV and the $\langle 110\rangle$ line connecting that site with the vacant site center increases with uniaxial compression from zero, at $\eta=1.0$, to $21.1^{\circ}$ at $\eta=1.275$. This increase corresponds to a displacement vector swing toward the [001] compression axis. For $1.275 \leqslant \eta \leqslant 1.425$, $\theta$ decreases from $21.1^{\circ}$ to zero and then falls to $-11.1^{\circ}$ at $\eta=1.5$. The negative progression corresponds to a swing from the $\langle 110\rangle$ site-to-site line direction toward 
the basal plane. The onset of the displacement vector rotation away from the stress axis toward the basal plane at $\eta=1.275$ correlates closely with the compressiun, $\eta=1.286$, at which the SV-OP becomes the stable vacancy configuration. The SV-OP displacement field pattern changes from the so-called crossed-axis pattern, exemplified in Figs. 44-46, to an alternating sign [101] shear pattern, exemplified by Figs. 47 and 48, as the unlaxial compression increases from 1.20 to 1.375. This compression range (centered on 1.30), in which the alternating sign, shear pattern develops, coincides with the compression range in which the SCV first-neighbor atom displacement vectors move away from the stress axis direction and toward the basal plane. In addition, it also coincides with the compression range in which the vacancy migration energy falls to a minimum at $\eta=$ 1.30, as shown in Fig. 22 .

It appears that a vacancy in a uniaxially compressed crystal is an extended defect for $r_{i}>1.20$. The alternating sign [101], shear displacement, field aspect of the vacancy displacement field is sufficiently developed for $\eta_{\gamma}>1.20$ that the displacement field nearly fills the computational cell. . Tables XLIV and and XLV describe the expansion of the vacancy displacement field that occurs with increasing uniaxial compression. Table XLIV pertains to the SCV and Table XLV to the SV-OP. Each of the uniaxial compression displacement field maps and tables in Sec. V pertains to the $y=4.5$ plane in the computational cell--the plane in which the vacancy was located. Tables XLIV and XLV list the numbers of sites in a $y=4.5$ plane that are in the vacancy GE1\% and GE3\% displacement fields. In the case of the SCV, these numbers include the vacant site, site 225 , and in the case of the SV-OP, they include vacant sites 225 and 275. For $\eta>1.20$, the fraction of the $\mathrm{y}=4.5$ plane sites in either the GE1\% or GE3\% displacement field is a good estimate of the fraction of all sites in the comp:tational cell in either the GE1\% or GE3\% dis-' acement field. These fractions are enclosed with parentheses in Tables XLIV and XLV.

Tables XLIV and XLV, together with the displacement field map figures for uniaxial compression $\eta>1.2$ clearly show that the vacancy displacement field fills the computational cell for $\eta>1.2$. As such, the volume of the vacancy displacement field region is 400-500 av, and the vacancy behaves like an extended defect (that is, its migration cannot be characterized by characterizing the movement of a single core atom). Part of the extended defect character indicated by the calculations may be an artifact of using periodic boundary conditions, 
Number of atoms in the $y=4.5$ plane in the GE1\% and GE3\% displacement fields of an SCV at site 225 as a function of uniaxial compression, $n$.

\section{Compression}

1.0

1.20

1.25

1.30

1.375

1.414

1.50

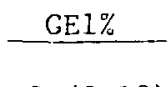

$5(0.10)$

$18(0.36)$

$41(0.82)$

$49(0.98)$

$45(0.90)$

$37(0.74)$

$45(0.90)$

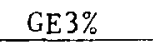

$$
0
$$

$8(0.16)$

$12(0.24)$

$26(0.52)$

$45(0.90)$

$13(0.26)$

$20(0.40)$
Number of atoms in the $y=4.5$ plane in the GE $1 \%$ and GE $3 \%$ displacement fields of an SV-OP as a function of uniaxial compression, $n$. Vacancies are at sites 225 and 275 .

\begin{tabular}{lllll} 
Compression & & $G E 1 \%$ & & GE3\% \\
\cline { 5 - 5 } \cline { 5 - 5 } 1.10 & & $15(0.30)$ & & $8(0.16)$ \\
1.20 & & $28(0.56)$ & & $11(0.22)$ \\
1.25 & & $39(0.78)$ & & $12(0.24)$ \\
1.375 & $50(1.00)$ & & $43(0.86)$ \\
1.414 & & $38(0.76)$ & & $19(0.38)$ \\
1.50 & & $42(0.84)$ & & $18(0.36)$
\end{tabular}

but in any event, we know that the vacancy displacement field cannot be encompassed by a 500-site computational cell for uniaxial compression $r_{1}>1.2$.

\section{REFERENCES}

1. Beeler, J. R., Jr., 1970, "The Role of Computer Experiments in Materials Research," in Advances in Materials Research, Vol. 4, H. Herman, Fd. (Interscience, New York), Pp. 295-476.

2. Beeler, J. R., Jr. and M. F. Beeler, 1979a, "Seli-Interstitials," U.S. Department of Energy report ORO-3912-102.

3. Beeler, J. R., Jr. and M. F. Beeler, 1979b, "Vacancies and Divacancies," U.S. Department of Energy report ORO-3912-103.

4. Beeler, J. R., Jr. and M. F. Beeler, 1979c, "Impurity Atoms," U.S. Department of Energy report ORO-3912-104.

5. Beeler, J. R, , Jr. and M. F. Beeler, 1979d, "Defect Migration," U.S. Department of Energy report ORO-3912-105.

6. Damask, A. C. and G. J. Dienes, 1963, Point Defects in Metals (Gordon and Breach, New York).

7. Guinan, M. W., R. N. Stuart, and R. J. Borg, 1977, "Fully Dynamic Computer Simulation of Self-Interstitial Diffusion in Tungsten," Phys. Rev. B15, 699.

8. Hirth, J. P., 1972, "Agenda Discussion: Point Defects," Appendix A, in Interatomic Potentials and Simulation of Lattice Defects, P. C. Gehlen, J. R. Beeler, Jr., and R. I. Jaffee, Eds. (Plenum Press, New York), pp. 456-458. 
9. Johnson, R. A. and W. D. Wilson, 1972, "Defect Calculations for FCC and BCC Metals," in Interatomic Potentials and Simulation of Lattice Defects, P. C. Gehlen, J. R. Beeler, Jr., and R. I. Jaffee, Eds. (Plenum Press, New York), pp. 301-319.

10. Vineyard, G. H., 1961, "General Introduction," in Discussions of the Faxaday Society, No. 31 , pp. 7-23.

11. Wilson, W. D. and C. L. Bisson, 1971, "Inert Gases in Solids: Interatomic Potentials and Their Influence on Rare-Gas Mobility," Phys. Rev. B3, 3984. 


\section{APPENDIX A \\ DISCONTINUITIES IN THE PERFECT CRYSTAL ENERGY \\ VERSUS COMPRESSION CURVE}

Compression causes each neighbor shell radius $r_{n}(\eta)$ to diminish as $\eta$ increases. Because the Lennard-Jones interatomic potential was truncated at $r_{\text {cut }}=$ $2.73823 \mathrm{hlc}(\mathrm{xy})$, a discontinuity in the crystal energy occurs whenever an $r_{n}(n)$ falls below $r_{\text {cut }}$ due to an increase in compression.

I. ISOTROPIC COMPRESSION

A. Fourth Neighbors

$$
\begin{aligned}
& r_{4}(\eta)=\left(0+\frac{4}{x^{2}}+\frac{4}{x^{2}}\right)^{1 / 2}, \text { where } x=n^{1 / 3} . \\
& r_{4}(\eta)^{2}=\frac{8}{x^{2}} .
\end{aligned}
$$

Setting $r_{4}(n)^{2}=r_{\text {cut }}^{2}$ gives $x=1.032940$ and $\eta=x^{3}=1.102111$. Hence, $r_{4}(n)$ will be less than $r_{\text {cut }}$ for all $n>\eta_{4}=1.102111$.

B. Fifth Neighbors

$$
\begin{aligned}
& r_{5}(n)=\left(0+\frac{1}{x^{2}}+\frac{9}{x^{2}}\right)^{1 / 2}, \text { where } x=n^{1 / 3} . \\
& r_{5}(n)^{2}=\frac{10}{x^{2}} .
\end{aligned}
$$

Setting $r_{5}(\eta)^{2}=r_{\text {cut }}^{2}$ gives $x=1.154862$ and $\eta_{5}=x^{3}=1.540246$

II. UNIAXIAL COMPRESSION

A. Fourth Neighbor Subshel1 (022)

$$
r_{4}(n)=\left(0+4+\frac{4}{\eta^{2}}\right)^{1 / 2} .
$$

Setting $r_{4}(n)^{2}=r_{\text {cut }}^{2}$ gives $2.738230^{2}-4=4 / \eta^{2} \cdot \eta_{4}=1.069365$. 
B. Fifth Neighbor Subshel1 (013)

$$
r_{5}(n)=\left(0+1+\frac{9}{n^{2}}\right)^{1 / 2}
$$

Setting $r_{5}(n)^{2}=r_{\text {cut }}^{2}$ gives $n_{5}=1.176887$ for (013) fifth neighbors.

C. Fifth Neighbor Subshel1 (031)

$$
\begin{aligned}
& r_{5}(n)=\left(0+9+\frac{1}{n^{2}}\right)^{1 / 2} . \\
& r_{5}(n)^{2} \geqslant 9>r_{\text {cut }}^{2} \text { for all } n .
\end{aligned}
$$

D. Sixth Neighbors

$$
\begin{aligned}
& r_{6}(n)=\left(4+4+\frac{4}{n^{2}}\right)^{1 / 2} . \\
& r_{6}(\eta)^{2} \geqslant 8>r_{\text {cut }}^{2} \text { for all } n .
\end{aligned}
$$

E. Seventh Neighbor Subshell (123)

$$
r_{7}\left(r_{i}\right)=\left(1+4+\frac{9}{n^{2}}\right)^{1 / 2}
$$

Setting $r_{7}(n)^{2}=r_{\text {cut }}^{2}$ gives $\eta_{7}=2.449832$.

F. Seventh Neighbor Subshel1 (132)

$$
r_{7}(n)=\left(1+9+\frac{1}{\eta^{2}}\right)^{1 / 2}>r_{\text {cut }} \text { for all } n
$$

G. Seventh Neighbor Subshell (231)

$$
r_{7}(n)=\left(4+9+\frac{1}{n^{2}}\right)^{1 / 2}>r_{\text {cut }} \text { for all } n
$$


H. Eighth Neighbor Subshel1 (004)

$$
r_{g}(\eta)=\left(\frac{16}{\eta^{2}}\right)^{1 / 2}
$$

Setting $r_{8}(\eta)^{2}=r_{\text {cut }}^{2}$ gives $\eta_{8}=1.460798$

Note: Distance is expressed in basal plane hlc(xy) in the above calculations. The basal plane hlc is dencted us hlc*.

\section{APPENDIX B}

\section{VACANCY RELAXATION COMPUTATION: INITIAL RANDOM ATOM DISPLACEMENTS}

This appendix gives the initial random atom displacements for the first neighbors of the vacancy in the site-centered vacancy computation for $\eta=1.0$. The displacements are expressed in hlc.

\begin{tabular}{|c|c|c|c|}
\hline Site & DX & DY & $\mathrm{DZ}$ \\
\hline 214 & 0.0068 & -0.0118 & -0.0021 \\
\hline 216 & 0.0071 & -0.0058 & -0.0035 \\
\hline 224 & -0.0031 & 0.0091 & -0.0127 \\
\hline 166 & -0.0039 & 0.0101 & -0.0039 \\
\hline 173 & -0.0121 & -0.0169 & 0.0003 \\
\hline 175 & -0.0061 & 0.0068 & -0.0100 \\
\hline 176 & 0.0002 & -0.0014 & -0.0090 \\
\hline 266 & -0.0062 & 0.0077 & -0.0123 \\
\hline 273 & -0.0042 & 0.0002 & -0.0052 \\
\hline 275 & 0.0156 & 0.0041 & -0.0022 \\
\hline 276 & 0.0159 & -0.0045 & -0.0012 \\
\hline
\end{tabular}

The average displacement component magnitude is 0.0068 and the average radial displacement is 0.0117 . 
Given an atom site with coordinates $(0,0,0)$, the relative $z$-coordinate of each neighboring atom site chaiges during uniaxial compression, but the relative $x$ - and $y$-coordinates do not change. If $z$ is the relative coordinate for compression $\eta=1.0$, then $z / \eta$ is the coordinate for compression $\eta \neq 1.0$. Figures C-1 - C-11 are neighbor she11 maps for the neighbors of a site marked $\square$. Neighboring sites are marked $O, \oplus$, and $\bullet$. The unit of distance is the half-lattice constant, $n$ is the neighbor shell index, and $C_{n}$ is the associated coordination number. OP means out-of-plane and IP means in-plane. The relative $x-$ and $y-$ coordinate of each site can be read from the map. Each nonzero relative $z$-coordinate is indicated as $\pm 1, \pm 2, \pm 3$, or \pm 4 . These maps are useful in visualizing how each neighbor stell is affected by uniaxial compression.

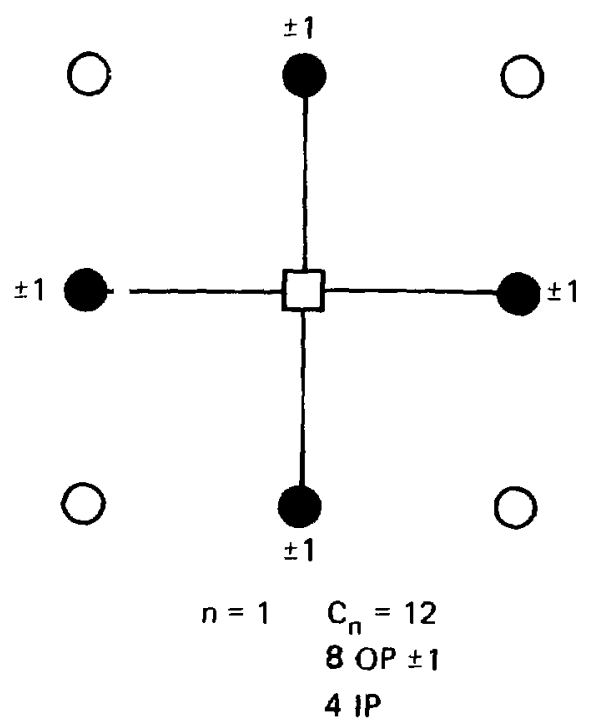

Fig. $\mathrm{C}-1$. First neighbors.

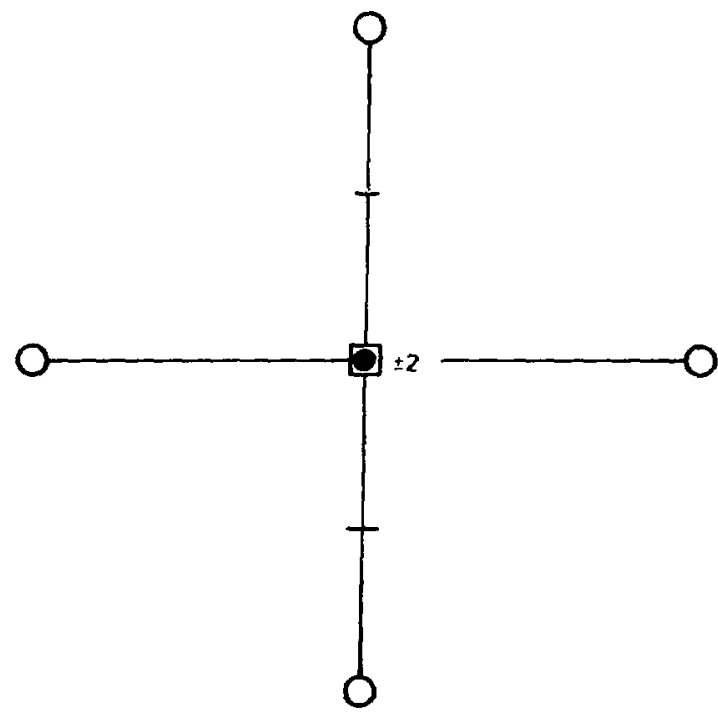

$$
\begin{array}{ll}
n=2 & C_{n}=6 \\
& 2 \text { OP } \pm 2 \\
& 4 \text { IP }
\end{array}
$$

Fig. $\mathrm{C}-2$. second neighbors. 


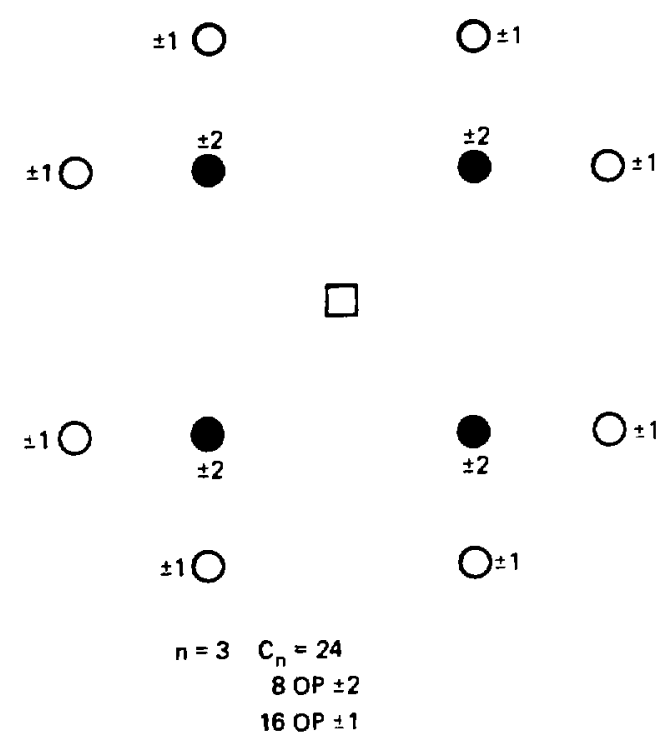

Fig. $\mathrm{C}-3$.

Third neighbors.

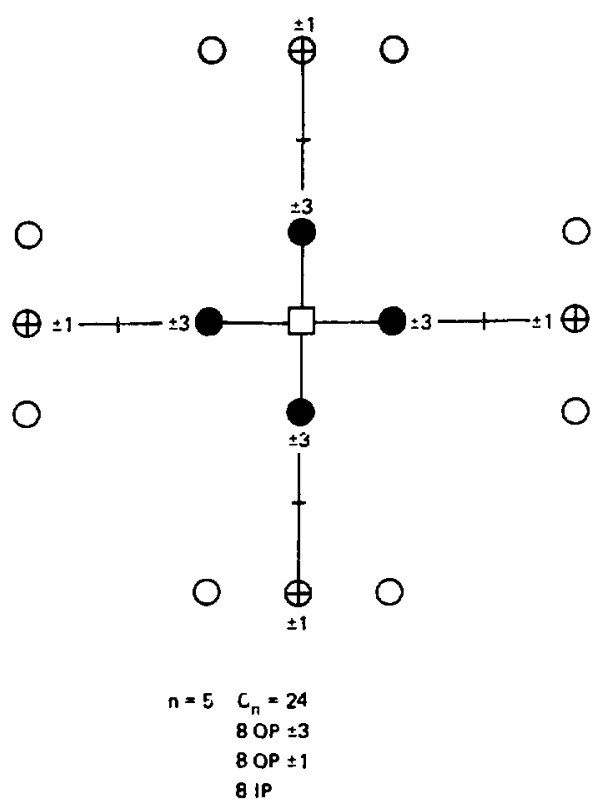

Fig. C-5.

Fifth neighbors.

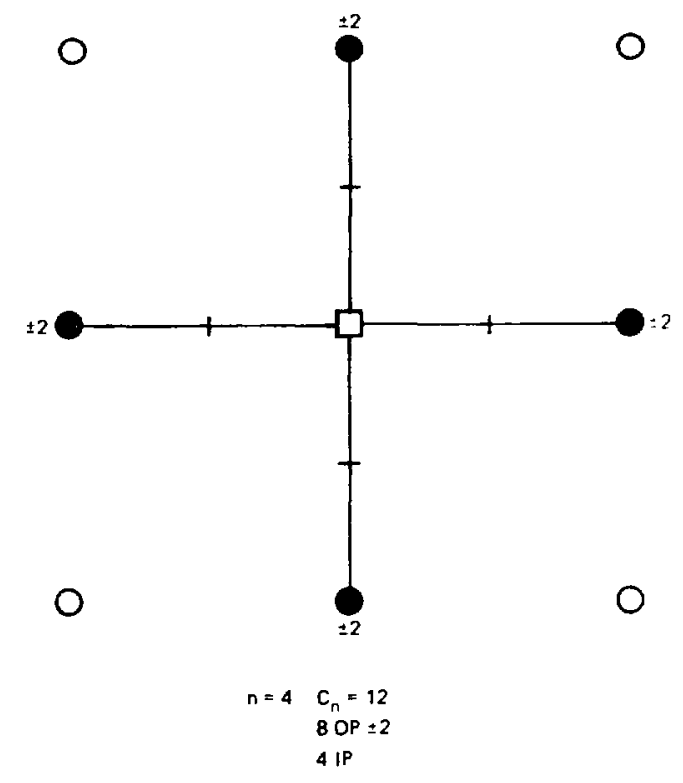

Fig. C-4.

Fourth neighbors.

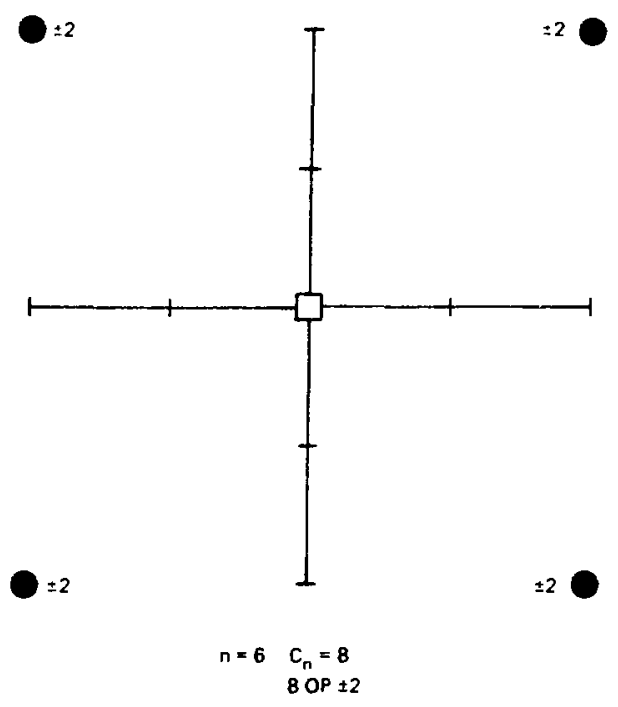

Fig. C-6.

Sixth neighbors. 


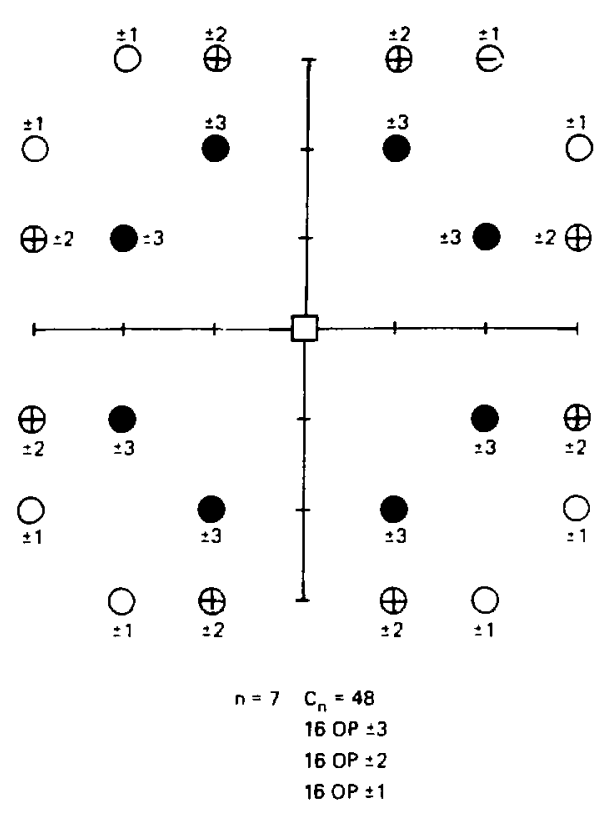

Fig. C-7.

Seventh neighbors.

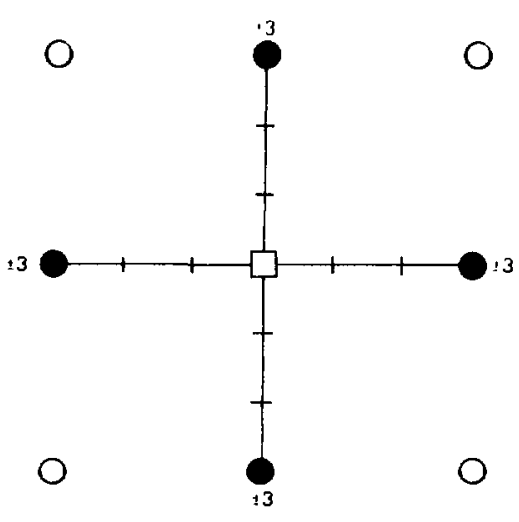

$$
\begin{aligned}
& n=9 \quad \text { [330] } \\
& C_{n}=12 \\
& B \text { OP } \pm 3 \\
& 4 \text { IP }
\end{aligned}
$$

Fig. C-9.

Ninth neighbors [330].

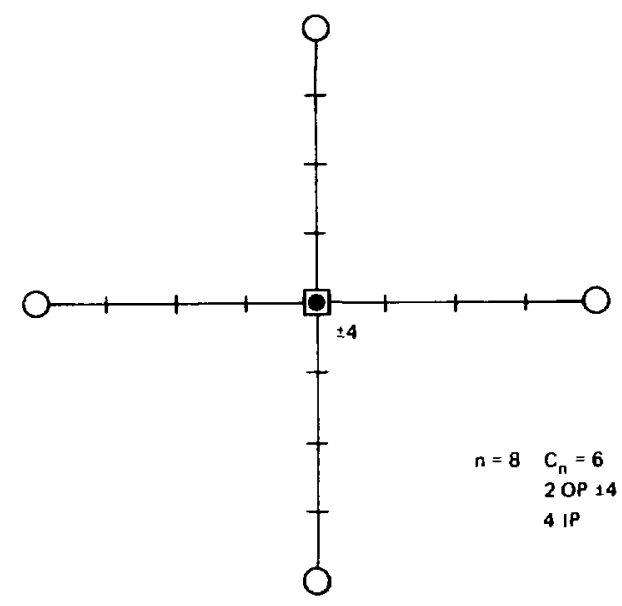

Fig. C-8.

Eighth neighbors.

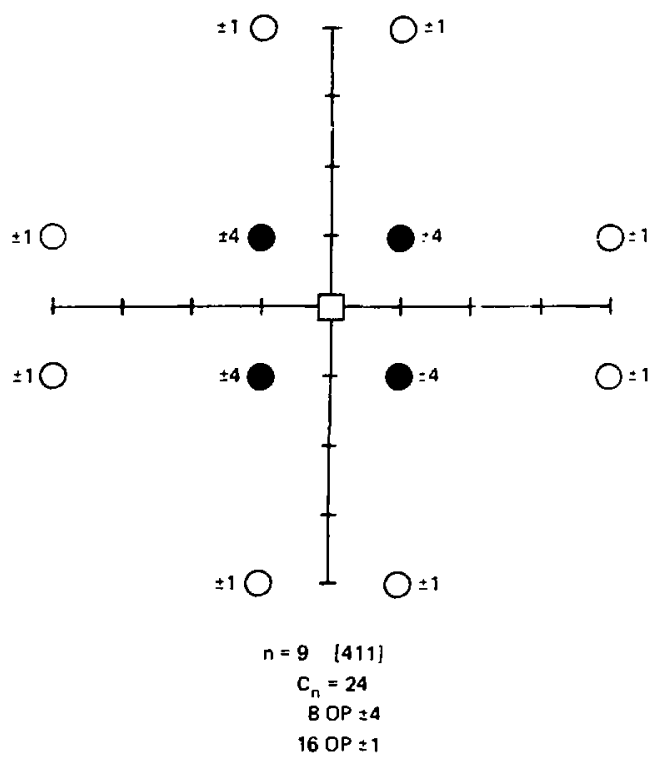

Fig. C-10.

Ninth neighbors [411]. 


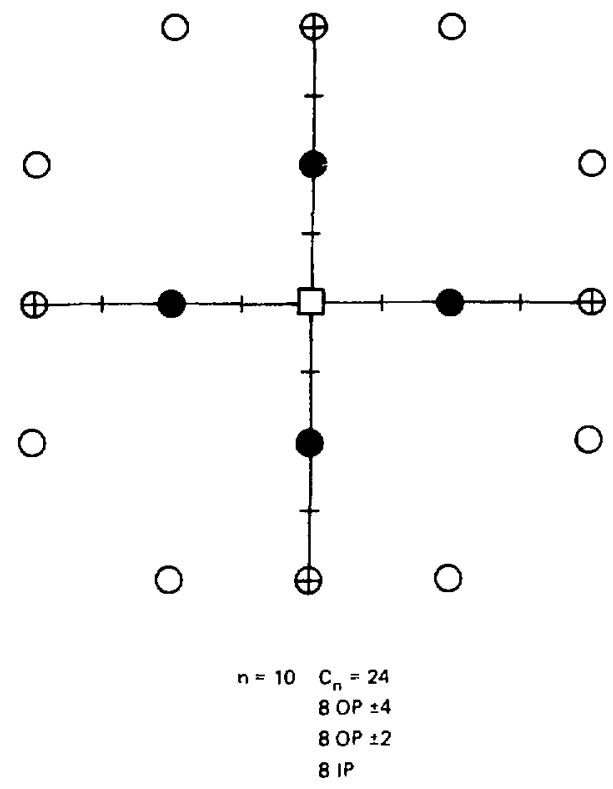

Fig. C-11.

Tenth neighbors. 